\title{
Cubillages of cyclic zonotopes
}

\author{
Vladimir I. Danilov* Alexander V. Karzanov ${ }^{\dagger} \quad$ Gleb A. Koshevoy
}

\begin{abstract}
Аннотация
This paper (written in Russian) presents a survey of new and earlier results on fine zonotopal tilings (briefly, cubillages) of cyclic zonotopes. The combinatorial theory of these objects is of interest in its own right and also has a connection to higher Bruhat orders, triangulations of cyclic polytopes, and Tamari-Stasheff posets applied in the study of Kadomtsev-Petviashvily equations, and etc.
\end{abstract}

Keywords: higher Bruhat order, Tamari-Stasheff poset, polycategory, rhombus tiling, separated sets, purity

Светлой памяти Владимира

Воеводского посвящается

\section{Содержание}

Введение

1 Зонотопы

2 Кубильяжи 7

3 Перегородки 9

4 Редукции и экспансии 11

5 Циклические (вполне положительные) векторные конфигурации 14

6 Мембраны

7 Кубы и флопы 16

${ }^{*}$ Central Institute of Economics and Mathematics of the RAS, 47, Nakhimovskii Prospect, 117418 Moscow, Russia; email: danilov@cemi.rssi.ru.

${ }^{\dagger}$ Central Institute of Economics and Mathematics of the RAS, 47, Nakhimovskii Prospect, 117418 Moscow, Russia; email: akarzanov7@gmail.com. Corresponding author.

$\ddagger$ The Institute for Information Transmission Problems of the RAS, 19, Bol'shoi Karetnyi per., 127051 Moscow, Russia, and HSE University, Moscow, Russia; email: koshevoyga@gmail.com. Supported in part by grant RSF 16-11-10075, and by Laboratory of Mirror Symmetry NRU HSE, RF Government grant, ag. № 14.641.31.0001. 
8 Капсиды и флипы

9 Порядок кубов в кубильяже 21

10 Порядок на кубильяжах капсида 23

11 Стэки и мембраны 26

12 Существование флипов 27

13 Мембраны в зонотопе 29

14 Допустимые порядки

15 Инверсии

16 Отношение разделенности 38

17 Разделенность и кубильяжи

18 Чистота и расширяемость 42

19 Случай $Z(6,4)$

20 Слабая разделенность 45

Дополнение 1. Поликатегорный взгляд на кубильяжи 48

Дополнение 2. Связь с триангуляциями и посетом Тамари-Сташева 50

Дополнение 3. Слабые мембраны 53

Дополнение 4. Доказательство ацикличности 56

\section{Введение}

Замощение фигур другими фигурами - излюбленная тема геометров. Вспомним кристаллические решетки и параллелоэдры, упаковки шаров, мозаики Пенроуза и связанные с этим ромбические тайлинги. Одно из направлений, интенсивно развивающееся в последние 30 лет, касается триангуляций т.н. циклических многогранников (см. [1] и обзор Рэмбо и Райнера [2] в сборнике [3]). Здесь мы хотим обсудить во многом параллельную теорию кубильяжей циклических зонотопов, то есть разбиений зонотопов на (комбинаторные) кубы.

Как это ни покажется странным, импульсы к рождению этой теории были не только геометрическими. Хотя зонотопальные (и кубические) разбиения издавна привлекали внимание геометров (см., например, работу [4, или исторический очерк в [5]), переворот вызвали две работы, скорее алгебраические или комбинаторные. Одна - работа Леклерка и Зелевинского [6] 1998 года - была связана с 
вопросом квазикоммутирования квантовых миноров и привела к изучению ромбических тайлингов зоногонов; обзор некоторых достижений на этом пути см. в статье [7]. Другая - статья Манина и Шехтмана [8] 1986 года (тоже мотивированная квантовой тематикой) - была посвящена обобщению слабого порядка Брюа на симметрической группе $S_{n}$; полученные упорядоченные множества $B(n, d)$ были названы высшими порядками Брюа. Несколько позже Воеводский и Капранов [9] дали интерпретацию этих порядков в терминах кубильяжей циклических зонотопов, частным (двумерным) случаем которых являются упомянутые выше ромбические тайлинги. Значительные продвижения в этом направлении были получены Циглером [10], Галашиным [11], и Галашинм и Постниковым [12] (см. также [13]).

Обо всем этом (включая наши собственные результаты) и предполагается рассказать в настоящей работе. Мы условно делим изложение на две тесно связанные части: геометрическую и комбинаторную (или теоретико-множественную). В первой рассказывается про зонотопы, кубические тайлинги (кубильяжи) зонотопов, вводятся разные полезные объекты в кубильяже: перегородки, тоннели, и т.п. Здесь же вводится основной рабочий инструмент - редукции и экспансии, незаменимые для проведения индуктивных рассуждений.

После введения этих общих понятий мы ограничиваем свое внимание исключительно случаем циклических (или - более правильно - вполне положительных) зонотопов $Z(n, d)$. Именнно кубильяжи таких зонотопов связаны с высшими порядками Брюа Манина-Шехтмана. При работе внутри фиксированного кубильяжа важную роль играет структура естественного порядка $\preceq$ на множестве его кубов. Когда же мы переходим к сравнению различных кубильяжей, тут на первый план выходит понятие флипа, некоторой локальной перестройки кубильяжа. Последнее проще всего объяснить на примере простейшего (после куба) зонотопа $Z(d+1, d)$, т. н. капсида. У него есть всего два кубильяжа, и замена одного кубильяжа на другой и называется флипом. В общем случае флип - это замена 'капсидного' фрагмента кубильяжа другим ('флипованным') фрагментом. Главный факт о кубильяжах состоит в том, что с помощью флипов можно перейти от любого кубильяжа зонотопа $Z(n, d)$ к любому другому.

Другое важное понятие - понятие мембран, гиперповерхностей специального вида внутри зоногона; мы показываем, что любую мембрану можно вписать в кубильяж.

Комбинаторная часть статьи связывает кубильяжи с системами подмножеств множества $[n]=\{1,2, \ldots, n\}$, индексирующего вектора, порождающие циклический зонотоп $Z(n, d)$. Имеется два способа сделать это. Первый рассматривает кубильяжи с точки зрения их спектров. Каждый кубильяж $\mathcal{Q}$ зонотопа $Z(n, d)$ ( $d$ - это размерность зонотопа, тогда как $n$ - число 'направляющих' векторов) задает систему $S p(\mathcal{Q})$ подмножеств в $[n]=\{1,2, \ldots, n\}$, то есть подмножество в $2^{[n]}$. Сет-система $S p(\mathcal{Q})$ однозначно определяет кубильяж $\mathcal{Q}$. Этот результат поднимает вопрос: какие сет-системы соответствуют кубильяжам, потому что ответ на него позволяет заменить геометрию комбинаторикой. Найденный Галашиным и Постниковым [12] ответ состоит в том, что такие сет-системы обладают т.н. свойством $(d-1)$-разделенности и при этом они максимальны по размеру (равному $\left.\left(\begin{array}{c}n \\ \leq d\end{array}\right)\right)$. Если максимальность по размеру можно заменить максимальностью 
по включению, то говорят о чистоте соответствующего отношения. Фундаментальное исследование вопросов чистоты было проведено в [12. Однако чтение этой статьи затруднено тем, что авторы работали с произвольными (не обязательно циклическими) зонотопами, и даже с ориентированными матроидами, и потому использовали усложненную матроидную терминологию и технику. Мы же сознательно ограничиваемся циклическим случаем, что делает (на наш взгляд) изложение более простым и доступным. В случаях $d=2$ и 3 свойство чистоты было доказано в [6] и [11], соответственно. В случае $d \geq 4$ ответ отрицателен; для этого мы подробно обсуждаем ситуацию в случае $n=d+2$, а также переносим отсутствия чистоты на большие $n$ и $d$.

Другой переход от геометрии к комбинаторике подсказывает отображение типов. С каждым кубом кубильяжа можно связать его mun, некоторое подмножество размера $d$ в множестве $[n]$. И это задает отображение (биективное, как несложно показать) кубильяжа в множество $\left(\begin{array}{c}{[n]} \\ d\end{array}\right)$ подмножеств размера $d$ в $[n]$. Пользуясь этой биекцией, можно перенести естественный порядок $\preceq_{\mathcal{Q}}$ с $\mathcal{Q}$ на $\left(\begin{array}{c}{[n]} \\ d\end{array}\right)$. Оказывается, что перенесенный порядок тоже однозначно определяет кубильяж $\mathcal{Q}$. Тем самым можно вместо кубильяжей работать с классом т.н. допустимых порядков на 'дискретном' грассманиане $G r([n], d)=\left(\begin{array}{c}{[n]} \\ d\end{array}\right)$. Так мы возвращаемся к истоку теории - определению высших порядков Брюа как допустимых порядков на грассманиане $\left(\begin{array}{c}{[n]} \\ d\end{array}\right)$. На геометрическом языке высший порядок Брюа $B(n, d)$ - это порядок на множестве $\mathbf{Q}(n, d)$ кубильяжей зонотопа $Z(n, d)$, или, эквивалентно, на множестве $\mathbf{M}(n, d)$ мембран в зонотопе $Z(n, d+1)$.

Кубильяжи имеют многочисленные связи с другими объектами математического мира. Например, известна их связь с уравнениями Кадомцева-Петвиашвили, идущая пока в основном через триангуляции циклических политопов. Уже отмечалось, что происхождение теории было мотивировано уравнениями Замолодчикова. Кубильяжи дают также естественный пример поликатегорий. Кубильяжи и триангуляции связаны с представлениями колчанов и конечномерных алгебр, см., например, работу [24]. О некоторых таких 'внешних' связях кубильяжей мы рассказывыаем кратко в Дополнениях. Здесь мы уже, как правило, не приводим точные определения и результаты, указывая лишь общие контуры связи. Таких Дополнений четыре. Это связь с поликатегориями, связь с триангуляциями (и через них с уравнениями К-П), рассказ о слабых мембранах, обобщающих понятие мембран. Туда же мы отнесли и доказательство теоремы об ацикличности из основного текста.

\section{ЧАСТЬ ПЕРВАЯ, ГЕОМЕТРИЧЕСКАЯ}

Здесь излагаются основные геометрические понятия и факты про кубильяжи зонотопов. Комбинаторый (теоретико-множественный) взгляд будет дан во второй части. Мы стараемся иллюстрировать все на 2-мерных кубильяжах (ромбических тайлингах), иногда рисовать 3-мерные картины. 


\section{1 Зонотопы}

Естественно начать с напоминаний про зонотопь 11 и введения терминов. Кубильяж - это, грубо говоря, правильное заполнение (разбиение) выпуклой фигуры 'кубами', а точнее говоря - параллелоэдрами.

Что же такое зонотоп? Фиксируем вещественное векторное пространство $V$ (размерности $d>0$ ) и некоторый конечный набор $\mathbf{V}=\left(v_{1}, \ldots, v_{n}\right)$ из $n$ векторов в нем. Зонотопом $Z=Z(\mathbf{V})$ (порожденным набором $\mathbf{V}$ ) называется сумма по Минковскому $n$ отрезков $\left[0, v_{i}\right]$. Иначе говоря, $Z$ состоит из точек $z$ вида $\sum_{i} \alpha_{i} v_{i}$, где $0 \leq \alpha_{i} \leq 1$ для любого $i=1, \ldots, n$. Можно также сказать, что зонотоп - это проекция 'единичного' $n$-мерного куба, когда базисные вектора пространства $\mathbb{R}^{n}$ отправляются в $v_{i}$. Это выпуклое тело, симметричное относительно точки (центра) $\sum v_{i} / 2$.

Зонотопом мы называем и любой сдвиг зонотопа. Заменяя, если нужно, вектора $v_{i}$ на $-v_{i}$, можно считать, что все они 'смотрят в одну сторону', если угодно - 'вверх' по отношению к некоторой 'горизонтальной' гиперплоскости. Такая замена меняет зонотоп на сдвинутый. Но зато теперь наш зонотоп имеет 'нижнюю' (корневую) вершину 0 и 'верхнюю' вершину $v_{1}+\ldots+v_{n}$.

Простейший пример зонотопа - это куб (точнее - параллелоэдр, но мы для краткости именуем его кубом). Это когда система $v_{1}, \ldots, v_{n}$ образует базис пространства $V$ (в этом случае $n=d$ ). Далее мы будем заниматься заполнениями зонотопов кубами.

Довольно легко описать грани зонотопа (которые тоже зонотопы меньшей размерности; см. [5, гл. 7] о кодировке граней знаковыми векторами). Мы еще более облегчим эту задачу. Во первых, мы будем считать, что система $\mathbf{V}$ находится в общем положении, то есть что $n \geq d$ и что любые $d$ векторов из системы $\mathbf{V}$ образуют базис пространства $V$. Далее это предположение всюду подразумевается, в основном по той причине, что мы будем заниматься кубильяжами $Z$. Во вторых, мы ограничимся описанием только фасет (граней коразмерности 1) зонотопа $Z$. Понятно, что фасеты такого 'общего' зонотопа будут кубами размерности $d-1$.

Чтобы задать фасету, мы фиксируем произвольные $d-1$ вектор $w_{1}, \ldots, w_{d-1}$ (подсистему $\mathbf{W})$ в нашей системе $\mathbf{V}$. Пусть $H_{\mathbf{W}}$ - гиперплоскость в $V$, натянутая на $\mathbf{W}$. Она делит оставшиеся точки-вектора $\mathbf{V}-\mathbf{W}$ на две части, скажем, $\mathbf{V}_{+}$и $\mathbf{V}_{-}$. Это дает две (противоположные друг другу) фасеты зонотопа $Z$. Одна образована зонотопом-кубом $Z(\mathbf{W})$, укорененным в точке $\sum \mathbf{V}_{+}$, а другая - тем же зонотопом, но укорененным в точке $\sum \mathbf{V}_{-}$. И так получаются все фасеты. Отсюда видно, что

$$
\text { 'общий' зонотоп } Z \text { имеет } 2\left(\begin{array}{c}
n \\
d-1
\end{array}\right) \text { фасет. }
$$

Тут неявно предполагалось, что $d>1$; случай одномерного зонотопа несколько выпадает из общей картины по причине его тривиальности; это просто отрезок $\left[0, v_{1}+\ldots+v_{n}\right]$.

Стоит еще сказать о вершинах зонотопа и об их числе. Наверняка это хорошо известно, но нам не удалось найти хорошую ссылку. Поэтому приведем формулу,

\footnotetext{
${ }^{1}$ Имеется большая литература про зонотопы, включая книги [16, 5].
} 
а позже скажем о доказательстве.

(1.2) Число вершин $V(n, d)$ зонотопа $Z$ равно $2\left(\begin{array}{c}n-1 \\ \leq(d-1)\end{array}\right)=2\left(\left(\begin{array}{c}n-1 \\ d-1\end{array}\right)+\ldots+\left(\begin{array}{c}n-1 \\ 0\end{array}\right)\right)$.

Другая полезная вещь - это взгляд на зонотоп вдоль некоторого направления. Пусть направление задается некоторым ненулевым вектором $w$ в $V$ и проектирует (отображением $\pi_{w}$ ) пространство $V$ (и находящийся в нем зонотоп $Z$ ) на (фактор)пространство $V^{\prime}=V / \mathbb{R} w$. Снова тут удобно считать, что этот вектор $w$ находтся в общем положении относительно системы V. В этом случае каждая фасета $F$ зонотопа $Z$ инъективно проектируется в пространство $V^{\prime}$. А весь зонотоп $Z$ проектируется на зонотоп $Z^{\prime}=\pi_{w}(Z)=Z\left(\pi_{w}(\mathbf{V})\right)$. При этом граница $\partial Z^{\prime}$ (имеющая размерность $d-2$ ) является биективным образом некоторого (тоже $(d-2)$-мерного) подкомплекса границы $Z$, который можно назвать ободом (относительно этой проекции $\pi_{w}$ ). Обод делит (нестрого) границу $Z$ на две 'полусферы' - 'верхнюю' (мы представляем проекцию $\pi_{w}$ как вертикальную, снизу вверх) и 'нижнюю'.

Здесь удобно ввести терминологию видимых и невидимых фасет. Снова берем 'общий' вектор $w$ (вдоль которого мы смотрим на зонотоп $Z$ ). Пусть $F$ - фасета $Z$. Скажем, что эта фасета $F$ видимая (в направлении $w$ ), если для (относительно внутренней) точки $p$ этой фасеты точка $p-\varepsilon w$ не принадлежит $Z$ (для малого или любого $\varepsilon>0$ ); тогда для малого $\varepsilon>0$ точка $p+\varepsilon w$ принадлежит $Z$. Иначе говоря, точка $p$ первая в зонотопе при движении (из $-\infty$ в $+\infty$ ) по прямой $p+\mathbb{R} w$.

В этой теминологии видимые фасеты покрывают нижнюю полусферу зонотопа, а невидимые - верхнюю. Проекция верхней полусферы дает один кубильяж зонотопа $Z^{\prime}$, а нижнего - другой, симметричный верхнему. Мы еще вернемся к этой теме в следующем разделе.

Можно проектировать не только вдоль 'общих' направлений, но и, скажем, вдоль направления вектора $v_{i}$, обозначая эту проекцию как $\pi_{i}$. Проекция при $\pi_{i}$ зонотопа $Z$ снова будет зонотопом $Z^{\prime}=Z / v_{i}$, порожденным (в $V^{\prime}=V / \mathbb{R} v_{i}$ ) образами всех векторов $v_{1}, \ldots, v_{n}$, кроме $v_{i}$. Однако теперь обод будет не $(d-2)$-мерный, но $(d-1)$-мерный (и называется он зоной или поясом зонотопа). Отметим также, что сам $Z$ получается как сумма зонотопа $Z\left(\mathbf{V}-\left\{v_{i}\right\}\right)$ и отрезка $Z\left(v_{i}\right)=\left[0, v_{i}\right]$.

После введения этих понятий можно обратиться к доказательству формулы (1.2). Зонотоп $Z=Z(\mathbf{V})$ представляется как сумма зонотопа $\widetilde{Z}=Z\left(\mathbf{V}-\left\{v_{n}\right\}\right)$ и отрезка $\left[0, v_{n}\right]$. При этом его вершины делятся на видимые и невидимые. Но видимые вершины (относительно направления $v_{n}$ ) зонотопа $\widetilde{Z}$ те же, что видимые вершины зонотопа $Z$; аналогично для невидимых (точнее, видимых с противоположного направления). Единственная разница состоит в том, что в пером случае эти два множества не пересекаются, тогда как во втором - пересекаются, причем в точности по множеству вершин обода (зонотопа $\widetilde{Z}$ в направлении $v_{n}$.). Отсюда мы получаем соотношение

$$
V(n, d)=V(n-1, d)+V(n-1, d-1) .
$$

Учитывая правило Паскаля, остается только убедиться в справедливости формулы (1.2) в случаях $d=1$ и $d=n$. В первом случае правая часть равна $2\left(\begin{array}{c}n-1 \\ 0\end{array}\right)=2$, 
что согласуется с тем, что отрезок имеет две вершины. Во втором случае правая часть равна $2\left(\begin{array}{c}d-1 \\ \leq d-1\end{array}\right)=2\left(2^{d-1}\right)=2^{d}$, что согласуется с тем, что $d$-мерный куб имеет $2^{d}$ вершин.

\section{2 Кубильяжи}

Кубильяж (или кубический, гиперромбический, параллелоэдральный тайлинг) это правильное замощение (разбиение, paving, tiling) зонотопа (или другого многогранника) кубами. Точнее:

$K у б и л ь я ж ~ \mathcal{Q}$ зонотопа $Z$ - это множество $d$-мерных кубов (см. выше) $Q_{1}, \ldots, Q_{N}$ (называемых плитками, тайлами или просто кубами), покрывающих $Z$, причем выполнены два условия:

1) два куба могут пересекаться только по общей грани;

2) фасеты зонотопа $Z$ являются фасетами некоторых кубов из $\mathcal{Q}$.

Точнее, так определяется кубильяж для зонотопов размерности $d>1$. Кубильяж одномерного зонотопа по определению состоит из $n$ отрезков, конгруэнтных отрезкам $\left[0, v_{i}\right], i=1, \ldots, n$.

Гранъю кубильяжа считается грань некоторого куба из $\mathcal{Q}$.

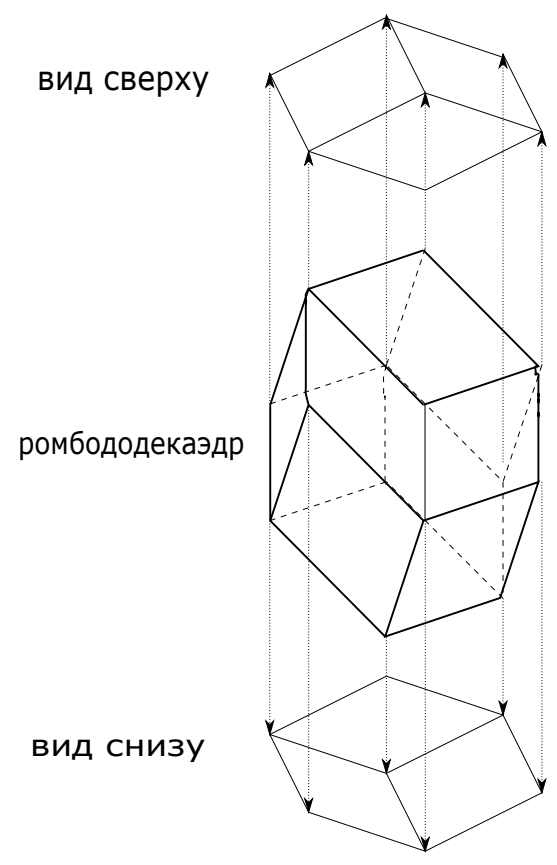

Рис. 1: Вид сверху и снизу на ромбододекаэдр

Пример. Пусть $Z$ - зонотоп $Z\left(v_{1}, \ldots, v_{n}\right)$, и мы проектируем его вдоль, скажем, направления $v_{n}$ (обозначая проекцию как $\pi$, см. рис. 11). Тогда, как объяснялось в предыдущем разделе, проекция фасет видимой части границы $Z$ дает кубильяж зонотопа $Z^{\prime}=Z\left(\pi\left(v_{1}\right), \ldots, \pi\left(v_{n-1}\right)\right)$. 
Часто на эту конструкцию смотрят в противоположном направлении, говоря, что система векторов $\mathbf{V}=\left\{v_{1}, \ldots, v_{n}\right\}$ - это 'одноэлементный лифтинг' системы $\mathbf{V}^{\prime}=\left\{\pi\left(v_{1}\right), \ldots, \pi\left(v_{n-1}\right)\right\}$ (с помощью вектора $\left.v_{n}\right)$. В такой терминологии одноэлементный лифтинг системы $\mathbf{V}^{\prime}$ дает кубильяж зонотопа $Z^{\prime}=Z\left(\mathbf{V}^{\prime}\right)$. Знаменитая теорема Бонэ-Дресса (см. [16] или [5]) в каком-то смысле утверждает обратное. Мы не приводим точную формулировку, поскольку это лежит в стороне от наших целей.

Полезным для понимания является следующий простой факт, который часто просто включают в определение кубильяжа.

Лемма 1 Пусть $Q$ - куб некоторого кубильяжа $\mathcal{Q}$ зототопа $Z=Z(\mathbf{V})$. Тогда любое ребро этого куба конгруэнтно некотоому отрезку $\left[0, v_{i}\right]$.

Доказательство. Можно считать, что $d>1$. Пусть $E$ - некоторое ребро куба $Q$. Если оно не лежит на границе зонотопа, вокруг него есть много кубов, ребра которых конгруэтны $E$. Переходя от одного такого куба к другому, мы рано или поздно выйдем на границу зонотопа. А тогда все следует из свойства 2) определения кубильяжа.

В частности, любой куб $Q$ кубильяжа конгруэнтен кубу-зонотопу $Z(\mathbf{W})$, где $\mathbf{W}$ - $d$-элементное подмножество в V. Множество индексов (или иветов; так мы понимаем элементы индексирующего множества $[n]=\{1, \ldots, n\})$ этого подмножества $\mathbf{W}$ называется типом этого куба. Иными словами, тип куба $Q$ - это подмножество $\left\{i_{1}, \ldots, i_{d}\right\}$ в $[n]$, такое что куб $Q$ есть сдвиг куба-зонотопа $Z\left(v_{i_{1}}, \ldots, v_{i_{d}}\right)$.

Аналогично понимается тип грани кубильяжа. В частности, тип ребра кубильяжа - одноэлементное подмножество $\{i\}$ в $[n]$. Мы ориентируем каждое ребро кубильяжа в направлении вектора $v_{i}$. Направленный путь из нижней вершины 0 в верхнюю вершину $v([n])$, идущий по ребрам кубильяжа, мы называем змейкой. Как мы увидим далее, в змейке ребро цвета $i$ встречается ровно один раз. Если $c(i)$ - цвет $i$-го ребра-стрелки некоторой змейки, то мы получаем биекцию $[n]$ в $[n]$, то есть перестановку множества $[n]$. Эту перестановку можно понимать также как линейный порядок на $[n]$, в котором минимальным элементом яаляется цвет первого ребра змейки.

Сопоставление кубу $Q$ кубильяжа $\mathcal{Q}$ его типа задает отображение $\tau=\tau_{\mathcal{Q}}$ : $\mathcal{Q} \rightarrow\left(\begin{array}{l}\mathbf{V} \\ d\end{array}\right)$ в множество $d$-элементных подмножеств $\mathbf{V}$.

Предложение 1 Отображение $\tau$ является биекцией. В частности, число кубов в любом кубильяюе равно $\left(\begin{array}{l}n \\ d\end{array}\right)$.

Мы докажем это утверждение в следующем разделе. А пока приведем простое

Следствие. Число вершин любого кубильяљса равно $\left(\begin{array}{c}n \\ \leq d\end{array}\right)$.

В самом деле, выберем некоторое общее направление проектирования ('смотрения') $w$. Из формулы (1.2) видно, что для каждого куба $Q$ кубильяжа все его вершины, кроме двух, лежат на ободе этого куба. И есть одна 'внутренняя' вершина на видимой половине границы $Q$ и, аналогично, одна 'внутренняя' вершина 
на невидимой половине. Обозначим эту последнюю, самую далекую он нас вершину куба как $h(Q)$. Легко понять, что отображение $h$ (из $\mathcal{Q}$ в множество вершин кубильяжа $\mathcal{Q}$ ) инъективно. А образ заметает все вершины, кроме вершин на видимой части $\partial_{-}(Z)$ границы $Z$. При проекции $\pi=\pi_{w}$ фасеты $\partial_{-}(Z)$ дают кубильяж зонотопа $Z^{\prime}=Z(\pi(\mathbf{V}), d-1)$, у которого, по предположению индукции, $\left(\begin{array}{c}n \\ d-1\end{array}\right)+\ldots+\left(\begin{array}{l}n \\ 1\end{array}\right)+\left(\begin{array}{c}n \\ 0\end{array}\right)$ вершин.

\section{3 Перегородки}

Пусть $\mathcal{Q}$ - некоторый кубильяж зонотопа $Z=Z(\mathbf{V})$, где $\mathbf{V}=\left\{v_{1}, \ldots, v_{n}\right\}$. В дальнейшем мы рассмотрим несколько интересных подмножеств в $\mathcal{Q}$ - перегородки, тоннели, гирлянды, стэки, капсиды ... Начнем с перегородок.

Определение. Перегородкой цвета $i \in[n]$ в кубильяже $\mathcal{Q}$ называется множество $\mathcal{P}_{i}$ кубов кубильяжа $\mathcal{Q}$, которые содержат цвет $i$ в своем типе. То есть

$$
\mathcal{P}_{i}=\{Q \in \mathcal{Q}, i \in \tau(Q)\} .
$$

(В некоторых работах перегородки называются также сечениями де Брюйна). Телом перегородки $\mathcal{P}_{i}$ называется объединение кубов из $\mathcal{P}_{i}$; это замкнутое подмножество в зонотопе $Z=Z(\mathbf{V})$, которое мы обозначаем как $\left|\mathcal{P}_{i}\right|$ (не путать с числом элементов множества $\mathcal{P}_{i}$, которое равно $\left.\left(\begin{array}{l}n-1 \\ d-1\end{array}\right)\right)$. Тело перегородки $\mathcal{P}_{i}$ выходит на границу зонотопа по зоне цвета $i$, см. раздел 1 .

Основная теорема о перегородках. Тело перегородки $\left|\mathcal{P}_{i}\right|$ устроено как произведение отрезка $\left[0, v_{i}\right]$ на некоторый диск, трансверсальный направлению $v_{i} u$ биективно проектирующийся на зонотоп $Z^{\prime}=\pi_{i}(Z)$ (где $\pi_{i}$ - проекция вдоль вектора $v_{i}$, см. выше).

Здесь для наглядности удобно считать вектор $v_{i}$ направленным вертикально вверх. В каждом кубе $P$ перегородки $\mathcal{P}=\mathcal{P}_{i}$ проведем 'среднее сечение' $M P$ (среднее по вертикальному направлению). Все такие локальные сечения склеиваются в $(d-1)$-мерный кубический комплекс $M \mathcal{P}$. Так как каждый куб $P$ представляется как сумма по Минковскому сечения $M P$ и трансверсального к нему вертикального отрезка $\left[-v_{i} / 2, v_{i} / 2\right]$, то и вся перегородка $\mathcal{P}$ есть сумма (по Минковскому) $M \mathcal{P}$ и отрезка $\left[-v_{i} / 2, v_{i} / 2\right]$. Более точно, естественное отображение

$$
M \mathcal{P} \times\left[-v_{i} / 2, v_{i} / 2\right] \rightarrow M \mathcal{P}+\left[-v_{i} / 2, v_{i} / 2\right]=|\mathcal{P}|
$$

является биекцией (и гомеоморфизмом). Отсюда легко понять, что при (вертикальной) проекции сечение $M \mathcal{P}$ локально гомеоморфно проектируется на $Z^{\prime}$. Так как зонотоп $Z^{\prime}$ стягиваем и, следовательно, односвязен, среднее сечение представляетися как сумма нескольких копий $Z^{\prime}$. На самом деле копия всего одна, что видно из того, что край этого комплекса $M \mathcal{P}$ образует 'среднее сечение' соответствующего обода (зоны цвета $i$ ) зонотопа $Z$.

Замечание. Перегородки (а еще лучше - их средние сечения) напоминают по своим свойствам гиперплоскости. Как и последние, они делят зонотоп на две области (до и после); кроме того, любые $d$ перегородок пересекаются по единственному 
кубу (а средние сечения - по единственной точке). Так что система всех перегородок выступает как некая замена арранжмента гиперплоскостей. Недаром вся эта теория у Манина и Шехтмана начиналась с аранжментов гиперплоскостей.

Проекция комплекса $M \mathcal{P}_{i}$ (или всей перегородки $\mathcal{P}_{i}$ ) дает кубильяж $\pi_{i}\left(\mathcal{P}_{i}\right)$ зонотопа $Z^{\prime}=\pi_{i}(Z)=Z / v_{i}$. Этот кубильяж (размерности $d-1$ ) зонотопа $Z^{\prime}$ можно обозначить как $\mathcal{Q} / v_{i}$. Вслед за Атанасиадисом [17] мы называем кубильяж $\mathcal{Q} / v_{i}$ сжатием (контрактацией) кубильяжа $\mathcal{Q}$ в направлении $v_{i}$, или сжатием по цвету $i$. Отметим, что при сжатии размерность кубильяжа уменьшается на единицу, как и число цветов.

Первое следствие основной теоремы про перегородки - это уже упоминавшееся утверждение про змейки. Напомним, что змейка - это направленный путь по ребрам-стрелкам кубильяжа из нижней (корневой) вершины зонотопа в верхнюю. Так вот, ивета ребер змейки биективны множеству $[n]$. То есть каждый цвет $i \in[n]$ встречается и при том один раз как цвет ребра змейки. В самом деле, перегородка $\mathcal{P}_{i}$ цвета $i$ делит зонотоп на три части - ниже $\mathcal{P}_{i}$, само $\mathcal{P}_{i}$, и выше $\mathcal{P}_{i}$. Наша змейка (при движении от корня к верхушке) обязательно пересекает перегородку, так что обязательно содержит стрелку цвета $i$. Причем пересекает ровно один раз, потому что после пересечения перегородки змейка попадает в верхнюю часть зонотопа и уже не может ее покинуть (в перегородке все стрелки цвета $i$ смотрят вверх).

Это следствие позволяет ввести важное понятие спектра (набора цветов) вершины кубильяжа, которое будет играть центральную роль в комбинаторной части. А именно, рассмотрим для вершины $v$ кубильяжа путь из 0 в $v$, идущий вверх по стрелкам-ребрам кубильяжа. Очевидно, что такой путь существует, быть может, не один. Этот путь - начальный кусок некоторой змейки, поэтому цвета его ребер образуют подмножество в $[n]$. Это подмножество не зависит от выбора пути; мы обозначаем его $s p(v)$ и называем спектром вершины $v$. Независимость от выбора пути видна из другого описания $s p(v)$ : цвет $i$ входит в $s p(v)$ тогда и только тогда, когда перегородка $\mathcal{P}_{i}$ проходит ниже вершины $v$ (то есть отделяет $v$ от корневой вершины 0). Заметим также, что $s p(v)$ не зависит и от кубильяжа $\mathcal{Q} ;$ в самом деле,

$$
v=\sum_{i \in s p(v)} v_{i}
$$

Наряду с перегородками можно рассмотреть в каком-то смысле дуальные объекты, называемые тоннелями в кубильяже $\mathcal{Q}$. Зафиксируем подмножество $D$ в $[n]$ размера $d-1$ и соберем вместе множество $\mathcal{T}_{D}$ кубов, типы которых содержат $D$ ('тоннель типа $D$ '). У каждого куба $Q$ из $\mathcal{T}_{D}$ есть две фасеты типа $D$. Пусть $F$ - такая фасета; тогда она либо фасета самого зонотопа $Z$, либо по этой фасете наш куб $Q$ смежен другому кубу $Q^{\prime}$, тоже из $\mathcal{T}_{D}$. Повторяя это с кубом $Q^{\prime}$, мы получаем соседний куб $Q^{\prime \prime}$ и так далее, пока не дойдем до фасеты зонотопа $Z$. (Эта конструкция уже встречалась при доказательстве Леммы 1.) Таким образом, тоннель представляет набор нескольких 'толстых путей' (или циклов) внутри кубильяжа. На самом деле легко понять, что тоннель - это связная цепочка кубов, идущих от одной (видимой) части границы $Z$ к другой, невидимой. Потому что 
каждый тоннель типа $D$ пересекает каждую перегородку $\mathcal{P}_{i}($ где $i \notin D)$ ровно по одному кубу (типа $D i$ ). И состоит ровно из $n-d+1$ куба.

Теперь обратимся к доказательству Предложения 1. Оно проводится индукцией по $d$; при $d=1$ утверждение тривиально. Пусть теперь $d>1$. Возьмем некоторую $d$-шку $D \subset[n]$ и покажем, что существует, и при том ровно один, куб $Q$ типа $D$ в нашем кубильяже $\mathcal{Q}$. Для этого выберем один из цветов (скажем, i) в $D$ и рассмотрим перегородку $\mathcal{P}_{i}$ цвета $i$. Пусть $\pi_{i}: Z \rightarrow Z^{\prime}=\pi_{i}(Z)-$ проекция вдоль вектора $v_{i}$. Как было сказано выше, проекция перегородки $\mathcal{P}_{i}$ дает кубильяж $\pi_{i}\left(\mathcal{P}_{i}\right)$ зонотопа $Z^{\prime}$. По индукции у него есть единственный куб $Q^{\prime}$ (размерности $d-1)$ типа $D-i$. Куб $Q$ из перегородки $\mathcal{P}_{i}$, проектирующийся на $Q^{\prime}$, имеет тип $(D-i) \cup\{i\}=D$. Это доказываент существование и единственность куба типа $D$.

Примерно так Шеппард [4] получал формулу для числа кубов кубильяжа зонотопа (см. Предложение 1).

\section{4 Редукции и экспансии}

Продолжим получать следствия структуры перегородок. Снова временно (и для наглядности) мы считаем, что вектор $v_{i}$ (произвольного цвета) идет вертикально вверх, так что мы смотрим на зонотоп $Z$ снизу вверх. Перегородка $\mathcal{P}=\mathcal{P}_{i}$ делит $Z$ на три части: собственно перегородку $\mathcal{P}$, то, что нестрого ниже $\mathcal{P}$ (включая нижнюю границу $\mathcal{P}$ ), что мы обозначим $Z_{-}$, и верхнюю половину $Z_{+}$.

Редукцией кубильяжа $\mathcal{Q}$ называется кубильяж $\mathcal{Q}_{-i}$ зонотопа $Z\left(\mathbf{V}-\left\{v_{i}\right\}\right)$, полученный следующим образом: нижнюю часть $Z_{-}$мы оставляем на месте (вместе со всеми ее кубами), а верхнюю часть $Z_{+}$сдвигаем вниз на вектор $-v_{i}$ (тоже вместе со всеми кубами). При этом перегородка $\mathcal{P}$ исчезает, а лучше сказать - сжимается на свою нижнюю границу, превращаясь в то, что позже мы назовем мембраной. Эту мембрану $\mathcal{M}=Z_{-} \cap\left|\mathcal{P}_{i}\right|$ мы называем швом (или рубцом), оставшимся после операции редукции. См. рис. 2 .
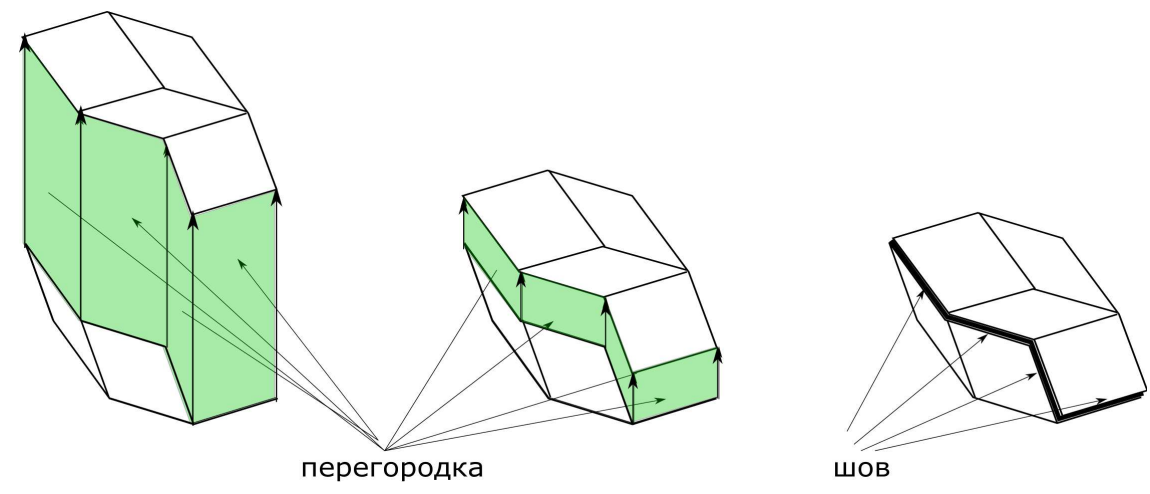

Рис. 2: Перегородка и ее постепенная редукция

Эта операция редукции обратима: если мы 'раздвинем' шов-мембрану $\mathcal{M}$ с 
помощью отрезка $\left[0, v_{i}\right]$ (отодвинув часть редуцированного кубильяжа, расположенную выше шва, на вектор $v_{i}$ ), мы вернемся к исходному кубильяжу $\mathcal{Q}$. Эту обратную операцию мы называем экспансией.

Вообще, если у нас есть кубильяж $\widetilde{\mathcal{Q}}$ зонотопа $\widetilde{Z}=Z\left(\mathbf{V}-\left\{v_{i}\right\}\right)$ и в нем мембрана $\mathcal{M}$ (то есть $(d-1)$-мерный подкомплекс кубильяжа $\mathcal{Q}$, который биективно проектируется при 'вертикальной проекции' на $\left.\widetilde{Z}^{\prime}=\pi(\widetilde{Z})\right)$, то мы можем 'раздвинуть' мембрану $\mathcal{M}$ на вектор $v_{i}$ и получить некоторый кубильяж $\mathcal{Q}$ зонотопа $Z$. Эту операцию мы называем экспансией мембраны $\mathcal{M}$ в кубильяже $\widetilde{\mathcal{Q}}$ в направлении $v_{i}$.

Следствие. Кубильяљ зонотопа $Z(\boldsymbol{V})$ определяется множеством его вершин.

Доказательство. Пусть $\mathcal{Q}$ и $\mathcal{R}$ - два кубильяжа зонотопа $Z(\mathbf{V})$ с одним и тем же множеством вершин. Выберем какой-то цвет, скажем, $n$, и произведем редукцию этого цвета в $\mathcal{Q}$ и $\mathcal{R}$. В результате мы получим два новых кубильяжа $\mathcal{Q}_{-n}$ и $\mathcal{R}_{-n}$ (зонотопа $Z\left(\mathbf{V}-\left\{v_{n}\right\}\right)$ ) и в каждом шов $S$ и $T$. Шов $S$ составлен из 'слившихся' вершин $v$ и $v^{\prime}$, таких что $s p\left(v^{\prime}\right)=s p(v) \cup\{n\}$; аналогично для шва $T$. Отсюда видно, что совпадают как эти два шва, так и множества вершин кубильяжей $\mathcal{Q}_{-n}$ и $\mathcal{R}_{-n}$. По индукции мы получаем совпадение самих кубильяжей $\mathcal{Q}_{-n}=\mathcal{R}_{-n}$. А исходные кубильяжи $\mathcal{Q}$ и $\mathcal{R}$ получаются $n$-экспансией общего шва $S=T$, так что они тоже совпадают.

Раз уж речь зашла о вершинах кубильяжа, то возникает вопрос - какие точки (или наборы точек) внутри зонотопа $Z=Z(\boldsymbol{V})$ могут быть вериинами некоторого кубильяжа этого зонотопа ('вписываются в кубильяж')? Одно требование очевидно (см. выше определение спектра): такая точки должна иметь вид $v(S)=\sum_{i \in S} v_{i}$ для некоторого подмножества $S \subset[n]$. Точки такого вида мы называем иельми, и это требование всюду далее предполагается выполненным. Мы еще вернемся к этому вопросу во второй части работы, а пока приведем один простой результат в этом направлении. А именно, мы утверждаем, что любая отдельно взятая целая точка вписывается в некоторый кубильяж. На самом деле верно даже большее: любой подзонотоп вписывается в некоторый кубильяљ. Более точно, множество вершин любого подзонотопа $T \subset Z$ вписывается в некоторый кубильяж зонотопа $Z$; здесь предполагается, что $T$ есть сдвиг на целую точку $v(S)$ зонотопа $Z(\mathbf{W})$, где $\mathbf{W} \subset \mathbf{V}$, и $S$ не пересекается с множеством индексов $\mathbf{W}$.

Предложение 2 Любой подзонотоп (и любой его кубилъяљ) $T$ зонотопа $Z=$ $Z(\boldsymbol{V})$ вписывается в некоторый кубильяљ $Z$.

Доказательство. Будем рассуждать индукцией по $n$ (или по размеру $\mathbf{V}-\mathbf{W}$ ). Пусть $t(T)$ - 'верхняя' вершина подзонотопа $T$. Предположим сначала, что эта вершина отлична от верхней вершины всего зонотопа $Z$. В этом случаем множество $s p(t(T))$ отлично от $[n]$; пусть $i$ - произвольный цвет, не принадлежащий $s p(t(T))$. Тогда подзонотоп $T$ лежит в зонотопе $Z^{\prime}=Z(\mathbf{V}-\{i\})$, и по индуктивному предполоржению вписывается в некоторый кубильяж $\mathcal{Q}^{\prime}$ зонотопа $Z^{\prime}=Z(\mathbf{V}-\{i\})$. Остается сделать экспансию по цвету $i$. См. рис. 3. 


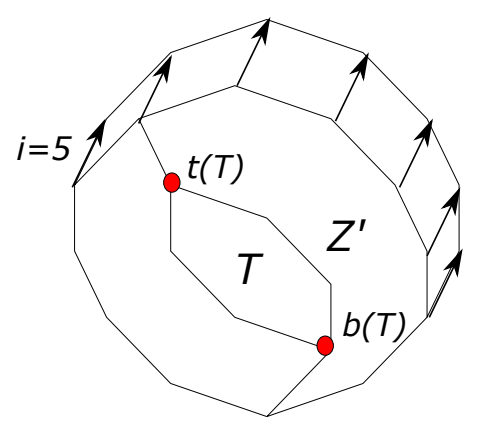

Рис. 3: подзоногон $T$ в зоногоне $Z(7,2)$

Таким образом, мы можем считать, что 'верхние' вершины у $T$ и $Z$ совпадают. Рассуждая симметрично, можно считать, что совпадают и 'нижние' (корневые) вершины. Но тогда $T$ и $Z$ совпадают, и утверждение тривиально верно.

Экспансию мембраны $\mathcal{M}$ можно производить не только в направлении вектора $v_{i}$, но и в любом 'близком' направлении $v_{i}^{\prime}$. Точнее, тут важна не близость, а только то, чтобы по отношению ко всем пластинкам (граням размерности $d-1$ ) мембраны $\mathcal{M}$ вектора $v_{i}$ и $v_{i}^{\prime}$ глядели в одну и ту же сторону. Раздвигая мембрану $\mathcal{M}$ в направлении $v_{i}^{\prime}$, мы получаем другой кубильяж другого зонотопа, но в каком-то смысле похожий, 'подобный' старому. Скажем об этом чуть подробнее.

Говоря о зонотопе $Z(\mathbf{V})$ и его кубильяжах, мы фиксировали векторную конфигурацию $\mathbf{V}$. Однако интуитивно ясно, что малые шевеления $\mathbf{V}$ не играют роли в строении кубильяжей. Скажем точнее. Пусть у нас есть две векторые конфигурации $\mathbf{V}=\left\{v_{1}, \ldots, v_{n}\right\}$ и $\mathbf{W}=\left\{w_{1}, \ldots, w_{n}\right\}$ (в одном и том же $d$-мерном пространстве $V$ и с одним и тем же числом векторов $n$ ). Скажем, что конфигурации $\mathbf{V}$ и $\mathbf{W}$

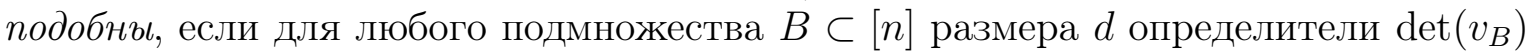
и $\operatorname{det}\left(w_{B}\right)$ имеют один и тот же знак (или что соответствующие базисы $v_{B}$ и $w_{B}$ имеют одну и ту же ориентацию).

Если конфигурации $\mathbf{V}$ и $\mathbf{W}$ подобны, то для любого кубильяжа $\mathcal{Q}$ зонотопа $Z(\mathbf{V})$ естественно строится 'подобный' кубильяж $\mathcal{Q}^{\prime}$ зонотопа $Z(\mathbf{W})$, который комбинаторно устроен так же, как $\mathcal{Q}$. А именно, пусть $Q$ - куб кубильяжа $\mathcal{Q} ;$ у него есть корневая вершина $v$ и тип $\tau(Q) \subset[n]$. Из начальной вершины 0 зонотопа $Z(\mathbf{V})$ в вершину $v$ ведет путь $P$ по стрелкам кубильяжа $Z(\mathbf{V})$. Проведем соответствующий путь $P^{\prime}$ в $Z(\mathbf{W})$ и конец его $v^{\prime}$ объявим образом вершины $v$. Ясно, что это построение не зависит от выбора пути $P$. Образ куба $Q$ - это куб того же типа, что и $Q$, растущий из вершины $v^{\prime}$. Так мы получаем новое множество $\mathcal{Q}^{\prime}$ кубов в $Z(\mathbf{W})$, и нужно лишь убедиться, что эти кубы не налезают друг на друга. А это достаточно проверить для соседних кубов. То есть если кубы $Q$ и $R$ кубильяжа $\mathcal{Q}$ соседние (по общей фасете $F$ ), то их образы $Q^{\prime}$ и $R^{\prime}$ (которые, очевидно, имеют общую фасету $F^{\prime}$ ) лежат по разные стороны от $F^{\prime}$.

Пусть фасета $F$ (то есть куб размерности $d-1$ ) имеет тип $\tau(F)$. Кубы $Q$ и $R$ получаются добавлением к векторам $v_{\tau(F)}$ каких-то векторов $v_{i}$ и $v_{j}$. Так как они лежат по разные стороны от $F$, то ориентации базисов $v_{\tau(F) i}$ и $v_{\tau(F) j}$ противоположны. Но тогда и ориентации базисов $w_{\tau(F) i}$ и $w_{\tau(F) j}$ противоположны, 
что и означает, что кубы $Q^{\prime}$ и $R^{\prime}$ лежат по разные стороны от $F^{\prime}$.

Это показывает, что строение (и разнообразие) кубильяжей зонотопа $Z(\mathbf{V})$ зависит только от ориентированного матроида, порожденного векторной конфигурацией V. Мы будем активно пользоваться этим простым замечанием при рассмотрении вполне положительных конфигураций.

\section{5 Циклические (вполне положительные) векторные конфигурации}

До сих пор конфигурации $\mathbf{V}$ векторов в пространстве $V$ были, фактически, произвольными (если не считать требования общего положения). Начиная с этого места мы ограничиваемся более специальными конфигурациями, которые обычно называются циклическими или вполне положительными. Здесь и далее мы будем считать, что $V=\mathbb{R}^{d}$ (с базисом $e_{1}, \ldots, e_{d}$ ); кроме того, теперь важную роль будет играть порядок индексации векторов $v_{i}$ (то есть, мы снабжаем множество индексов-цветов $[n]$ естественным порядком $1<2<\ldots<n)$.

Определение. Конфигурация векторов $\mathbf{V}=\left(v_{1}, \ldots, v_{n}\right)$ называется вполне положительной (или, не очень удачно, но более коротко, ииклической), если для любой (возрастающей) $d$-шки $i_{1}<i_{2}<\ldots<i_{d}$ из $[n]$ определитель матрицы $\operatorname{Mat}\left(v_{i_{1}}, \ldots, v_{i_{d}}\right)$, составленной из столбцов-векторов $v_{i_{j}}$, положителен.

Циклические конфигурации подобны между собой и определяются двумя числами $d$ и $n \geq d$. По этой причине циклический зонотоп $Z(\mathbf{V})$ обозначается далее просто как $Z(n, d)$, а множество его кубильяжей - как $\mathbf{Q}(n, d)$.

Удобный представитель такой конфигурации получается следующим образом. Отобразим прямую $\mathbb{R}$ в $\mathbb{R}^{d}$ с помощью отображения $\mathbf{v}_{d}, \mathbf{v}_{d}(t)=\left(1, t, t^{2}, \ldots, t^{d-1}\right)$. (Когда $d$ ясно из контекста, мы опускаем инлекс $d$ и говорим просто про $\mathbf{v}$. Образ прямой $\mathbb{R}$ при таком отображении называют кривой Веронезе или кривой моменmов.) Если мы теперь возьмем конечное подмножество $\left\{t_{1}<\ldots<t_{n}\right\}$ в $\mathbb{R}$, мы получаем (индексированную множеством $[n]$ ) векторную конфигурацию $\mathbf{V}$, состоящую из векторов $v_{i}=\mathbf{v}\left(t_{i}\right), i=1, \ldots, n$. Такая конфигурация вполне положительна, что видно из формулы для определителей Вандермонда.

Отметим два важных для дальнейшего свойства циклических конфигураций. Если мы выбрасываем какой-то элемент $k$ из $[n]$, конфигурация остается циклической. Так что мы можем говорить о проекциях $\pi_{k}: Z(n, d) \rightarrow Z([n]-\{k\}, d)$ и соответствующих редукциях кубильяжей (см. выше). И второе (применимое к конфигурациям Веронезе): если мы рассмотрим каноническую проекцию $\pi$ пространства $\mathbb{R}^{d}$ на $\mathbb{R}^{d-1}$ (проекция вдоль последнего базисного вектора $e_{d}$, или забывание $d$-ой координаты), то циклическая конфигурация переходит в циклическую. В частности, мы получаем проекцию $\pi: Z(n, d) \rightarrow Z(n, d-1)$, полезную для проведения индукции по $d$. Заметим еще, что эту проекцию $\pi$ (вдоль $d$-ой координатной оси) можно понимать как проекцию вдоль направления $\mathbf{v}(+\infty)$, потому что направление вектора $\mathbf{v}(t)$ при большом $t$ стремится к направлению вектора $e_{d}$. Неформальная же причина интереса циклических конфигураций объясняется 
тем, что кубильяжи циклических зонотопов тесно связаны с высшими порядками Манина-Шехтмана, о чем мы будем говорить в комбинаторной части работы.

Мы уже говорили про редукцию цветов для общих зонотопов. В случае циклических зонотопов особо важную роль играет редукция старшего цвета $n$. Дело в том, что шов, остающийся после такой редукции, является мембраной относительно проекции $\pi$ вдоль $d$-й координатной оси. Расскажем более обстоятельно об этом в следующем разделе.

\section{6 Мембраны}

Определение. Пусть $\mathcal{Q}$ - кубильяж (циклического) зонотопа $Z=Z(n, k)$. Meмбраной этого кубильяжа, или мембраной, вписанной в кубильяж $\mathcal{Q}$, называется $(d-1)$-мерный подкомплекс $\mathcal{M}$ в $\mathcal{Q}$, который биективно проектируется на зонотоп $Z(n, d-1)$ (при проекции $\pi$ вдоль последней $d$-ой координаты).

Множество мембран кубильяжа $\mathcal{Q}$ обозначается как $\mathbf{M}(\mathcal{Q})$.

Так что мембрана - это некоторая $(d-1)$-мерная пленка в $Z$ (идущая по граням кубильяжа $\mathcal{Q})$, гомеоморфная $(d-1)$-мерному диску, край которого - в точности обод зонотопа $Z$ относительно проекции $\pi$. Мембрана делит зонотоп на две части: нестрого до мембраны $\left(Z_{-}(\mathcal{M})\right)$ и нестрого после нее $\left(Z_{+}(\mathcal{M})\right)$. Проекция клеток мембраны $\mathcal{M}$ (ее $(d-1)$-мерных кубов, называемых пластинами) дает при этом кубильяж зонотопа $Z^{\prime}=Z(n, d-1)=\pi(Z)$, который мы обозначаем $\pi(\mathcal{M})$.

Априори не ясно, существуют ли мембраны вообще и много ли их. Позже мы увидим, что их достаточно много. А пока приведем пример двух универсальных мембран.

Пример 1. Мы уже говорили про это в разделе 2; давайте вернемся применительно к циклической ситуации. Рассмотрим видимую (в направлении $d$-ой координаты) часть границы $Z, \partial_{-} Z$. Очевидно, это мембрана (любого кубильяжа зонотопа $Z)$; ее проекция $\pi\left(\partial_{-} Z\right)$ дает кубильяж зонотопа $Z^{\prime}$, который мы называем стандартным (так именовались соответствующие ромбические тайлинги двумерного зоногона в [7]). Невидимая часть границы $\partial_{+} Z$ дает анти-стандартныи кубильяж зонотопа $Z^{\prime}$.

Универсальный способ получения мембран дает редукция старшего цвета. Пусть $\mathcal{Q}$ - кубильяж зонотопа $Z=Z(n, d)$. И пусть $\widetilde{\mathcal{Q}}=\mathcal{Q}_{-n}-$ кубильяж зонотопа $\tilde{Z}=Z(n-1, d)$, полученный редукцией цвета $n$. Тогда шов $\mathcal{S}$ от этой редукции является мембраной. В самом деле, замена вектора $v_{n}$ на $d$-й базисный вектор $e_{d}=(0, \ldots, 0,1)$ в $\mathbb{R}^{d}$ приводит к подобной векторной конфигурации. Поэтому (см. раздел 4) можно произвести экспансию шва $\mathcal{S}$ в направлении $e_{d}$ и получить кубильяж $\mathcal{Q}^{\prime}$, подобный $\mathcal{Q}$. А это значит (см. основную теорему из раздела 3 ), что шов $\mathcal{S}$ биективно проектируется на зонотоп $\pi(Z)=Z(n, d-1)$.

Напротив, имея мембрану $\mathcal{M}$ в кубильяже $\widetilde{\mathcal{Q}}$ зонотопа $\widetilde{Z}=Z(n-1, d)$, мы можем экспансировать эту мембрану в направлении нового вектора $v_{n}=\mathbf{v}\left(t_{n}\right)$, где $t_{n}>t_{n-1}$. Дело в том, что направление, задаваемое вектором $v_{n}$, также трансверсально мембране $\mathcal{M}$ (как и направление $\mathbf{v}(+\infty)$ ). Мы говорили об этом в разделе 
4 про подобные конфигурации. В результате получаем новый кубильяж $\mathcal{Q}$ зонотопа $Z=Z(n, d)$. 'Раздвинутая' мембрана $\mathcal{M}+\left[0, v_{n}\right]$ становится перегородкой старшего цвета $n$ в $\mathcal{Q}$. Редуцируя по этому цвету, мы возвращаемся к исходному кубильяжу $\widetilde{\mathcal{Q}}$.

Таким образом, пара $(\widetilde{\mathcal{Q}}, \mathcal{M})$, где $\widetilde{\mathcal{Q}}$ - кубильяж зонотопа $\widetilde{Z}=Z(n-1, d)$, а $\mathcal{M}$ - мембрана в $\widetilde{\mathcal{Q}}$, определяет кубильяж $\mathcal{Q}$ зонотопа $Z=Z(n, d)$. И на этом пути получаются все кубильяжи зонотопа $Z$. В самом деле, пусть $\mathcal{Q}$ - произвольный кубильяж $Z$. Возьмем в нем перегородку $\mathcal{P}$ цвета $n$ и редуцируем ее. Шов $\mathcal{S}$ от этой редукции, как уже говорилось, является мембраной для редуцированного кубильяжа $\widetilde{\mathcal{Q}}=\mathcal{Q}_{-n}$ редуцированного зонотопа $Z(n-1, d)$. Экспансия этой мембраны $\mathcal{S}$ возвращает нас к исходному кубильяжу $\mathcal{Q}$.

Итог этого обсуждения можно подвести следующим утверждением.

Предложение 3 Задать кубильяж зонотопа $Z(n, d)$ - то же самое, что задать пару $(\widetilde{\mathcal{Q}}, \mathcal{M})$, где $\widetilde{\mathcal{Q}}-$ кубильяљ зонотопа $Z(n-1, d)$, а $\mathcal{M}$ - мембрана для $\widetilde{\mathcal{Q}}$.

Или чуть иначе: существует естественное сюръективное отображение $\mathbf{Q}(n, d) \rightarrow$ $\mathbf{Q}(n-1, d)$; слой его над любой точкой $\mathcal{Q} \in \mathbf{Q}(n-1, d)$ совпадает с множеством $\mathbf{M}(\mathcal{Q})$ мембран для $\mathcal{Q}$.

Пример 2. Стандартный кубильяж зонотопа $Z(n, d)$ получается из стандартного же кубильяжа зонотопа $Z^{\prime}=Z(n-1, d)$ с помощью 'задней' мембраны $\partial_{+}\left(Z^{\prime}\right)$ (невидимой части границы $Z^{\prime}$ ). См. рис. 7, где $d=2$.

В самом деле, мы определяли стандартный кубильяж зонотопа $Z(n, d)$ как образ 'передней' (видимой) мембраны $\partial_{-} Z(n, d+1)$ зонотопа $Z(n, d+1)$. Но как выглядит последняя? Зонотоп $Z(n, d+1)$ получается из зонотопа $Z(n-1, d+1)$ прибавлением отрезка $\left[0, \mathbf{v}_{d+1}(n)\right]$. Соответственно, его видимая граница состоит из видимой границы $\partial_{-}$зонотопа $Z(n-1, d+1)$ плюс фасеты вида $F+\left[0, \mathbf{v}_{d+1}(n)\right]$, где $F$ пробегает множество невидимых (в направлении координаты $d$ ) фасет этой видимой границы $\partial_{-}$. При проекции вдоль координаты $d+1$ первая половина превращается в стандартный кубильяж зонотопа $Z^{\prime}=Z(n-1, d)$, а фасеты $F-$ в невидимые фасеты этого зонотопа $Z^{\prime}$, образуя в совокупности невидимую часть границы зонотопа $Z^{\prime}$. Поэтому вторая половина проектируется в точности на экспансию (по цвету $n$ ) задней (невидимой) мембраны зонотопа $Z^{\prime}$.

Аналогично, антистандартный кубильяж получается экспансией по цвету $n$ передней (видимой) мембраны $\partial_{-} Z^{\prime}$ антистандартного кубильяжа зонотопа $Z^{\prime}=$ $Z(n-1, d)$.

\section{7 Кубы и флопы}

Рассмотрим более обстоятельно случай куба, зонотопа $C=Z(d, d)$. У него есть всего две мембраны - видимая (передняя) $\partial_{-} C$ и невидимая (задняя) $\partial_{+} C$, которые при проекции $\pi$ дают стандартный и антистандартный кубильяжи зонотопа $Z(d, d-1)$. Из подсчета числа вершин обода (см. (1.2)) видно, что на передней 
мембране имеется ровно одна 'внутренняя' вершина $t$ (назовем ее $x в о с т о м)$, как и на задней одна 'внутренняя' вершина $h$ (назовем ее головой).

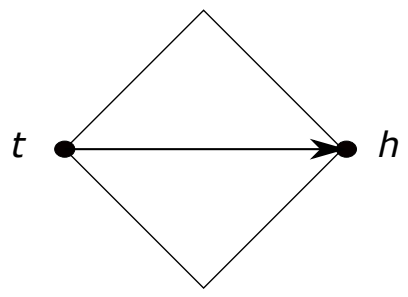

$d=2$

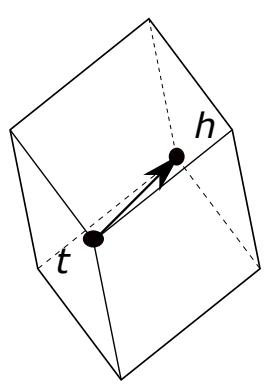

$d=3$

Рис. 4: Хвост и голова

Скажем более конкретно о спектрах этих вершин. Для этого вернемся к разделу 1 , где описывались фасеты зонотопа. Аналогично можно поступить и здесь, для описания вершин $t$ и $h$. Напомним, что вектора $v_{1}, \ldots, v_{d}$ задавались числами $t_{1}<\ldots<t_{d}$ и мы полагали $v_{i}=\mathbf{v}\left(t_{i}\right)$. Возьмем некоторые перемежающие их числа $s_{1}, \ldots, s_{d-1}$, так что $t_{1}<s_{1}<t_{2}<\ldots<t_{d-1}<s_{d-1}<t_{d}$. И в качестве линейного функционала на пространстве $\mathbb{R}^{d}$ возьмем $\operatorname{det}\left(\mathbf{v}\left(s_{1}\right), \ldots, \mathbf{v}\left(s_{d-1}\right), \bullet\right)$. Заметим, что этот функционал положителен на векторах $v_{d}$ и $e_{d}$. Он положителен также на векторах $v_{i}$, когда $i$ имеет ту же четность, что и $d$, и отрицателен на векторах $v_{i}$, когда $i$ имеет противоположную с $d$ четность. Отсюда видно, что головная вершина $h$ есть сумма векторов $v_{i}$, таких что $d-i$ четно, тогда как хвостовая вершина $t$ есть сумма векторов $v_{i}$, таких что $d-i$ нечетно. Так что $s p(h)=\{d, d-2, \ldots\}$, тогда как $s p(t)=\{d-1, d-3, \ldots\}$. Например, в случае $d=3$ (см. рис. 4) $s p(t)=2, s p(h)=13$ (как и далее, 13 обозначает множество $\{1,3\}$ ). Очевидно, что в объединении эти множества-спектры дают все $[d]$.

Стрелку, идущую из хвоста $t$ в голову $h$, будем назвать хордой куба $C$. В случае четного $d$ хорда идет горизонтально, тогда как в при нечетном $d$ она поднимается на 1 при каждом шаге, как видно из рис. 4. Здесь впервые начинает проявляться небольшое различие между четными и нечетными $d$.

После расмотрения изолированного куба обратимся к общей ситуации. Пусть $\mathcal{Q}$ - кубильяж зонотопа $Z=Z(n, d)$, и $Q$ - некоторый куб этого кубильяжа с хвостом $t_{Q}$, головой $h_{Q}$ и хордой $t_{Q} \rightarrow h_{Q}$. Если мы проделаем это с каждым кубом кубильяжа, то эти хорды дадут структуру направленного графа на множестве вершин кубильяжа $\mathcal{Q}$. Этот граф состоит из путей-нитей, соединяющих (внутренние) вершины передней мембраны $\partial_{-} Z$ с (внутренними же) вершинами задней мембраны $\partial_{+} Z$. Каждый куб оказывается 'нанизанным' на свою хорду, а кубы, нанизанные на пути, выглядят как 'гирлянды'. Считая, что вершины обода соединены сами с собой путем длины 0, мы получаем интересную (и слабо изученную) 'гирляндную' биекцию множества вершин передней мембраны в множество вершин задней мембраны зонотопа $Z$.

Разумеется, можно не ограничиваться только граничными мембранами. Если 


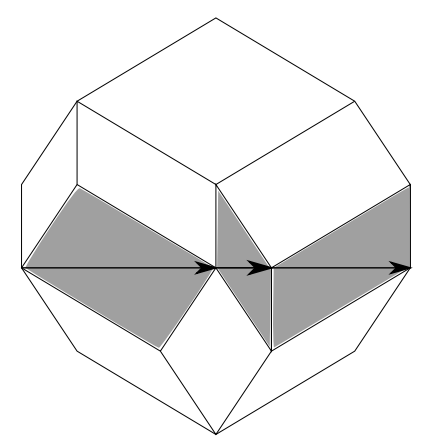

Рис. 5: Гирлянда в зоногоне $Z(5,2)$

$\mathcal{M}$ и $\mathcal{M}^{\prime}$ - две произвольные мембраны (одного и того же кубильяжа), то каждая гирлянда 'протыкает' каждую из мембран ровно одит раз. Тем самым мы получаем 'гирляндную' биекцию между множеством вершин $\mathcal{M}$ и множеством вершин $\mathcal{M}^{\prime}$. Это лишний раз показывает, что число вершин у каждой мембраны такое же, как у стандартной. На самом деле мы получаем даже больше: при четном $d$ у всех мембран зонотопа $Z(n, d)$ на каждой высоте одно и то же число вершин. Или: при нечетном $d$ у любого кубильяжа зонотопа $Z(n, d)$ число вершин на каждом этаже одно и то же.

Пример. Проиллюстрируем гирляндную биекцию на примере стандартного кубильяжа занотопа $Z(4,3)$. Как уже говорилось в предыдущем разделе, такой кубильяж получается из куба $C=Z(3,3)$ экспансией по цвету 4 задней стороны этого куба. Задняя сторона $\partial_{+} C$ состоит из трех пластинок-ромбов, так что стандартный кубильяж $Z(4,3)$ состоит из четырех кубов: исходного $C$ и трех 'экспансированных' из этих пластинок. Мы изобразили все это на рис. 5 , где для наглядности вынули и слегка отодвинули первый куб $C$.

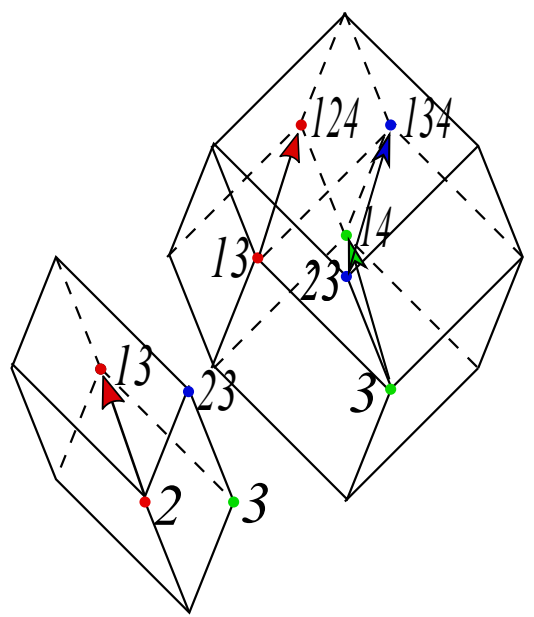

Рис. 6: Гирляндное отображение для стандартного кубильяжа зонотопа $Z(4,3)$

Передняя сторона (или мембрана) зонотопа $Z(4,3)$ содержит, кроме перифе- 
рийных (лежащих на ободе), три вершины со спектрами 2,3 и 23. Так как гирляндное отображение $\gamma_{S}$ тривиально действует на ободе, нам нужно понять - куда при отображении $\gamma_{S}$ переходят эти три вершины 2,3 и 23. Глядя на рисунок, мы видим, что 2 переходит сначала в точку 13, которая затем переходит в точку 124. Точка 3 переходит в 14, а точка 23 - в 134. Окончательно,

$$
\gamma_{S}:\left\{\begin{array}{rl}
2 & \mapsto 124 \\
3 & \mapsto 14 \\
23 & \mapsto 134
\end{array} .\right.
$$

Действуя аналогично, можно убедиться, что гирляндное отображение $\gamma_{A}$ (для антистандартного кубильяжа $Z(4,3))$ устроено так:

$$
\gamma_{A}:\left\{\begin{array}{c}
2 \mapsto 14 \\
3 \mapsto 134 \\
23 \mapsto 124
\end{array} .\right.
$$

Последнее, впрочем, можно увидеть и из следующего общего соображения. Произвольный зоногон $Z$ симметричен относительно своего центра. Если обозначить эту симметрию как $\alpha$, мы для любого кубильяжа $\mathcal{Q}$ можем рассмотреть симметричный ему кубильяж $\alpha \mathcal{Q}$ того же зонотопа $Z$. При этом стандартный кубильяж переходит в антистандартный, и наоборот. Пусть $S$ обозначает вершины зоногона на передней стороне $Z$, а $A$ - вершины на задней стороне. Инволюция $\alpha$ осуществляет биекцию $S$ на $A$; подмножество $X$ переходит в свое дополнение $[n]-X$.

Предложение 4 Если $\gamma_{\mathcal{Q}}: S \rightarrow A$ - гирляндная биекиия, связанная с кубильяжем $\mathcal{Q}$, mо $\gamma_{\alpha \mathcal{Q}}=\alpha^{-1} \gamma_{\mathcal{Q}} \alpha$.

Доказательство. При инволюции $\alpha$ каждая хорда куба $Q$ переходит в противоположно направленную хорду куба $\alpha Q$.

Пусть $\mathcal{M}$ - мембрана в некотором кубильяже $\mathcal{Q}$. Представим, что некоторый куб $Q$ кубильяжа $\mathcal{Q}$ касается мембраны всей своей видимой границей $\partial_{-} Q$. (Ниже мы увидим, что такая ситуация не исключительна и встречается постоянно.) В этом случае можно образовать новую мембрану $\mathcal{M}^{\prime}$, которая всюду совпадает с $\mathcal{M}$, за исключением того, что этот фрагмент $\partial_{-} Q$ заменяется новым фрагментом $\partial_{+} Q$. Иначе говоря, если мембрана $\mathcal{M}$ обходила куб $C$ спереди, то теперь она обходит его сзади. Мы говорим, что мембрана $\mathcal{M}^{\prime}$ получена из $\mathcal{M}$ с помощью повышающего флопа (а $\mathcal{M}$ получена из $\mathcal{M}^{\prime}$ понижающим флопом).

Это можно выразить чуть иначе. Пусть $\mathcal{Q}_{-}(\mathcal{M})$ обозначает набор кубов кубильяжа $\mathcal{Q}$, которые расположены до мембраны $\mathcal{M}$. Тогда $\mathcal{Q}_{-}\left(\mathcal{M}^{\prime}\right)$ получается добавлением к $\mathcal{Q}_{-}(\mathcal{M})$ ровно одного куба $Q$. Так что при повышающих флопах мембрана движется 'от нас', захватывая по очереди по одному кубу кубильяжа, пока не дойдет до 'задней' границы зонотопа. 


\section{8 Капсиды и флипы}

После обстоятельного рассмотрения куба естественно перейти к следующему по сложности случаю зонотопа $Z=Z(d+1, d)$. Такой зонотоп (или подзонотоп в большем зонотопе $Z(n, d))$ будем для краткости называть капсидол2.

Капсид представляется как проекция $(d+1)$-мерного куба $\widehat{Z}=Z(d+1, d+1)$. Проекция передней мембраны этого куба дает стандартный кубильяж капсида $Z$. А проекция 'хвоста' $t_{\widehat{Z}}$ дает единственную 'центральную' (не лежащую на ободе) вершину стандартного кубильяжа, которую мы обозначим как $c^{s t}$. Ее спектр $s p\left(c^{s t}\right)=\{d, d-2, \ldots\}$. Эта вершина со всех сторон окружена кубами стандартного кубильяжа капсида и является вершиной каждого куба этого кубильяжа. Симметрично невидимая мембрана содержит 'центральную' вершину $c^{a n}$ антистандартного кубильяжа капсида со спектром $\{d+1, d-1, \ldots\}$. Например, при $d=2 s p\left(c^{s t}\right)=2, s p\left(c^{a n}\right)=13$; при $d=3 s p\left(c^{s t}\right)=13, s p\left(c^{a n}\right)=24$.

В силу Следствия из Раздела 4 мы получаем, что капсид имеет только два кубильяжа, описанных выше. Подведем итог:

Предложение 5 Капсид $Z(d+1, d)$ допускает всего два кубилъяжа - стандартный и антистандартный. Стандартный кубильяж характеризуется тем, что

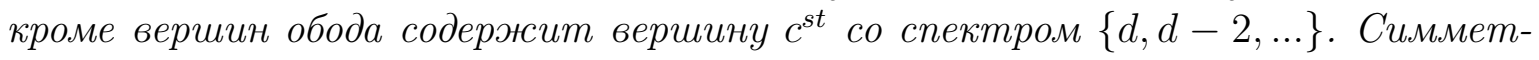
рично, антистандартный кубилъяж характеризуется тем, что кроме вериин

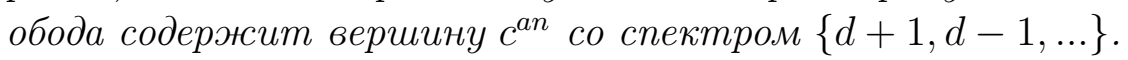

Утверждение про два кубильяжа капсида можно получить также из Предложения 3. Последнее говорит, что задать кубильяж капсида $Z(d+1, d)$ - это то же самое, что задать мембрану в кубе $C=Z(d, d)$. Но у куба есть всего две мембраны: видимая часть границы $\partial_{-} C$ и невидимая часть границы $\partial_{+} C$. Соответственно, у капсида $Z(d+1, d)$ есть два кубильяжа. Один получается $(d+1)$-экспансией задней мембраны $\partial_{+} C$; это стандартный кубильяж. Другой (антистандартный) получается путем экспансии передней мембраны $\partial_{-} C$. См. рис. 7.

Предположим теперь, что у нас имеется некоторый кубильяж $\mathcal{Q}$ произвольного зонотопа $Z(n, d)$, а в нем фрагмент вида $Z(d+1, d)$ (то есть граница этого капсида является подкомплексом комплекса $\mathcal{Q}$ ). Ограничение кубильяжа $\mathcal{Q}$ на этот капсид $Z(d+1, d)$ является стандартным или антистандартным кубильяжем. Замена кубильяжа этого фрагмента противоположным кубильяжем называется флипом ( повышающим, когда стандатрный фрагмент заменяется антистандартным, и понижающим в противном случае). Флип меняет кубильяж $\mathcal{Q}$ на некоторый новый кубильяж $\mathcal{Q}^{\prime}$, отличающийся от $\mathcal{Q}$ лишь локально, внутри капсида.

Связь флипов с флопами, рассмотренными в предыдущем разделе, очевидна. Предположим: что кубильяж $\mathcal{Q}$ зонотопа $Z(n, d)$ получен как проекция мембраны $\mathcal{M}$ в кубильяже $\widehat{\mathcal{Q}}$ "большего"зонотопа $\widehat{Z}=Z(n, d+1)$. И мы делаем (скажем, повышающий) флоп с этой мембраной, заменяя ее мембраной $\mathcal{M}^{\prime}$. Тогда проекция $\mathcal{Q}^{\prime}$ новой мембраны $\mathcal{M}^{\prime}$ получается применением (повышающего) флипа к кубильяжу $\mathcal{Q}^{\prime}$.

\footnotetext{
${ }^{2}$ Термин взят из вирусологии. Так называется белковая оболочка вируса, обычно полиэдральная (типа ромбододекаэдра, то есть $Z(5,3)$ ).
} 

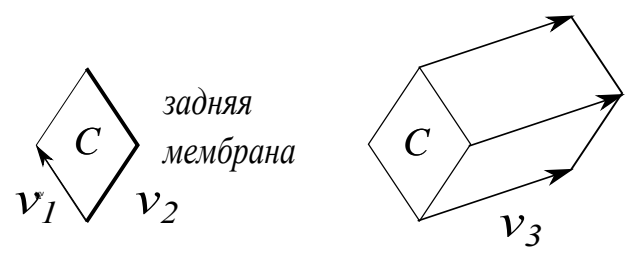

стандартныій

кубильяж
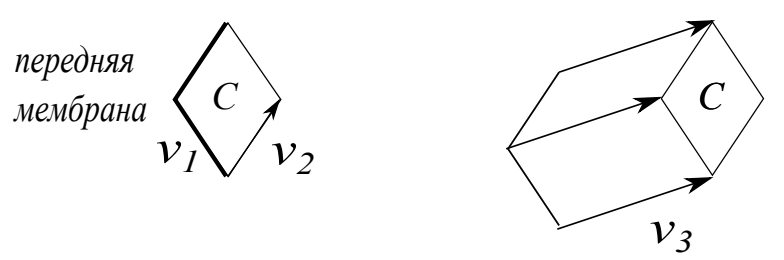

антистандартный

кубильяжс

Рис. 7: экспансия мембран куба

Как мы увидим ниже, флипов много, хотя бы в том смысле, что от любого кубильяжа $\mathcal{Q}$ можно добраться до стандартного кубильяжа $\mathcal{Q}_{s t}$ с помощью понижающих флипов. Однако независимо от этого факта можно ввести понятие порлдка на множестве $\mathbf{Q}(n, d)$. А именно, $\mathcal{Q} \leq \mathcal{Q}^{\prime}$, если от кубильяжа $\mathcal{Q}$ до кубильяжа $\mathcal{Q}^{\prime}$ можно добраться серией повышающих флипов. Чтобы показать, что флипов 'много', полезно ввести важное понятие естественного порядка на множестве кубов произвольного кубильяжа.

\section{9 Порядок кубов в кубильяже}

Пусть $\mathcal{Q}$ - кубильяж (циклического) зонотопа $Z(n, d) \subset \mathbb{R}^{d}$. Оказывается, можно частично упорядочить некоторым естественным образом кубы этого кубильяжа. Говоря грубо - по росту $d$-й координаты. Но более правильно - по тому, располагается ли один куб за другим при смотрении в направлении $d$-й координаты.

Пусть $Q$ - некоторый куб кубильяжа $\mathcal{Q}$, и $F$ - его фасета. Скажем, что фасета $F$ куба $Q$ видимая (или освещенная), если существует прямая (в $\left.\mathbb{R}^{d}\right)$, параллельная координатному вектору $e_{d}$, которая сначала пересекает $F$ и лишь после этого входит в куб $Q$. Противоположная (к $F$ в $Q$ ) фасета называется невидимой или затененной. Очевидно, что из $2 d$ фасет куба половина видимые, а половина затенненные.

Пусть теперь $Q$ и $Q^{\prime}$ - два куба кубильяжа $\mathcal{Q}$. Скажем, что куб $Q$ непосредственно предшествует кубу $Q^{\prime}$ (и пишем $Q \prec Q^{\prime}$ ), если $Q$ и $Q^{\prime}$ соседние по фасете $F$, которая невидима для $Q$ и видима для $Q^{\prime}$. Так на $\mathcal{Q}$ возникает бинарное отношение $\prec$ (или $\prec_{\mathcal{Q}}$, если быть точнее).

Рисунок 8 подсказывает следующее обобщение: 


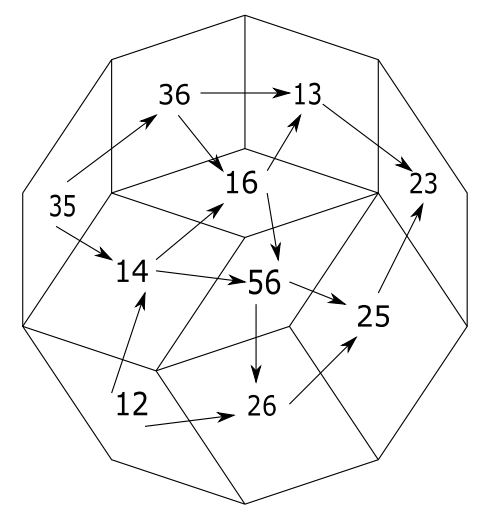

Рис. 8: Отношение $\prec$ на кубильяже зоногона $Z(5,2)$.

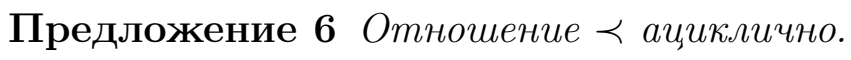

Это утверждение, как и следующая Лемма о реверсе доказываются в Дополнении 4. Лемма относится к следующей ситуации. Пусть $\mathcal{P}=\mathcal{P}_{n}$ - перегородка старшего цвета $n$ в кубильяже $\mathcal{Q}$ зонотопа $Z=Z(n, d)$. Редукция этой перегородки дает кубильяж $\widetilde{\mathcal{Q}}$ зонотопа $\widetilde{Z}=Z(n-1, d)$ и шов-мембрану $\mathcal{M}$ в $\widetilde{\mathcal{Q}}$. Проектируя $\mathcal{M}$ вдоль $d$-ой координатной оси $e_{d}$, мы получаем кубильяж $\mathcal{Q}^{\prime}=\pi(\mathcal{M})$ зонотопа $Z^{\prime}=Z(n, d-1)$.

Пусть теперь $Q$ и $R$ - два куба из перегородки $\mathcal{P}$, связанные отношением непосредственного предшествования, $R \prec Q$. При редукции кубы $Q$ и $R$ превращаются в пластинки-фасеты $\gamma(Q)$ и $\gamma(R)$ мембраны $\mathcal{M}$; их образы при проекции $\pi$ дают кубы $Q^{\prime}$ и $R^{\prime}$ кубильяжа $\mathcal{Q}^{\prime}$. Очевидно, что $Q^{\prime}$ и $R^{\prime}$ тоже соседние в кубильяже $\mathcal{Q}^{\prime}$, и мы можем сравнить их отношением непосредственного предшествования $\prec^{\prime}$ в кубильяже $\mathcal{Q}^{\prime}$.

Лемма 2 (о реверсе). Если кубы $R$ и $Q$ лежат в перегородке $\mathcal{P}$ u $R \prec Q$, то $Q^{\prime} \prec^{\prime} R^{\prime}$.
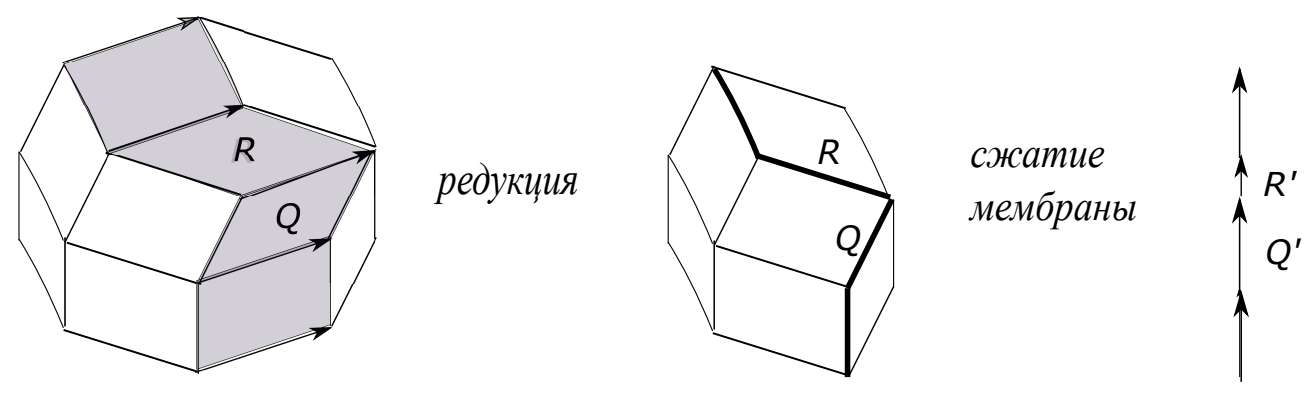

Рис. 9: На рисунке слева для кубов $R$ и $Q$ из перегородки выполнено $R \prec Q$. Справа нарисован кубильяж $\mathcal{Q}^{\prime}$, полученный сжатием перегородки. Для соотвествующих кубов $R^{\prime}$ и $Q^{\prime}$ выполнено противоположное соотношение $Q^{\prime} \prec R^{\prime}$. 
Непосредственным следствием Леммы о реверсе является то, что ограничение отношения $\prec$ на перегородку $\mathcal{P}$ (цвета $n$ ) противоположсно отношению $\prec^{\prime} \partial л я$ кубильяжа $\mathcal{Q}^{\prime}$ (здесь мы отождествляем множество $\mathcal{P}$ с $\mathcal{Q}^{\prime}$ через $P \mapsto \gamma(P) \mapsto P^{\prime}$ ).

Определение. Eстественным порядком на кубильяже $\mathcal{Q}$ называется рефлексивно-транзитивное замыкание отношения $\prec$; мы обозначаем его $\preceq$ или $\preceq_{\mathcal{Q}}$.

По определению это отношение $\preceq$ является предпорядком; нетривиальность в том, что оно является порядком, то есть антисимметрично $\left(Q \preceq Q^{\prime}\right.$ и $Q^{\prime} \preceq Q$ влекут $\left.Q=Q^{\prime}\right)$. Именно это и дает Предложение 6 .

Пример 1. Пусть $Q$ и $Q^{\prime}$ - два куба из одного туннеля, и $Q^{\prime}$ идет после $Q$ при движении от видимой границы зонотопа к невидимой. Тогда $Q \preceq Q^{\prime}$.

Пример 2. Пусть куб $Q$ 'частично затеняет' $Q$ ', то есть имеется прямая, параллельная координатному вектору $e_{n}$, которая протыкает (пересекает по внутренней точке) куб $Q$ раньше, чем $Q^{\prime}$. Тогда $Q \preceq Q^{\prime}$. В самом деле, надо рассмотреть цепочку кубов $Q_{1}, \ldots, Q_{k}$, которые эта прямая пересекает по пути от $Q$ к $Q^{\prime}$. Тогда $Q \prec Q_{1} \prec \ldots \prec Q_{k} \prec Q^{\prime}$.

Отметим, что мы могли бы начать с этого более сильного отношения затенения и (после транзитивного замыкания) получить то же отношение $\preceq$.

Пример 3. Пусть куб $Q^{\prime}$ располагается непосредственно после куба $Q$ в некоторой гирлянде (то есть голова $Q$ совпадает с хвостом $Q^{\prime}$, см. раздел 7 ). Тогда $Q$ затеняет $Q^{\prime}$ и по предыдущему примеру $Q \preceq Q^{\prime}$.

Пусть $\mathcal{Q}$ - кубильяж, а $\mathcal{Q}_{-i}-$ редукция $\mathcal{Q}$ по цвету $i$. Как множество кубов $\mathcal{Q}_{-i}$ отождствляется с $\mathcal{Q}-\mathcal{P}_{i}$, так что можно рассматривать $\mathcal{Q}_{-i}$ как подмножество в $\mathcal{Q}$.

Предложение 7 Ограничение порядка $\preceq$ на $\mathcal{Q}_{-i}$ сильнее, чем порядок $\varliminf_{-i}$ на $\mathcal{Q}_{-i}$.

Иначе говоря, если для кубов $Q$ и $R$ из редуцированного кубильяжа $\mathcal{Q}_{-i}$ выполнено соотношение $Q \preceq_{-i} R$, то $Q \preceq R$.

Для доказательства мы должны проверить, что если $Q$ непосредственно предшествует $R$ в $\mathcal{Q}_{-i}$, то $Q \preceq R$. Соотношение $Q \prec_{-i} R$ означает, что кубы $Q$ и $R$ соседние в $\mathcal{Q}_{-i}$ по фасете $F$, причем $Q$ располагается раньше. Возможны два случая. Первый - фасета $F$ не является пластиной шва $\mathcal{S}$ редукции. Тогда $Q$ и $R$ соседние уже в $\mathcal{Q}$ и все очевидно. Второй - фасета $F$ принадлежит шву. Но тогда она получилась редукцией цвета $i$ в кубе $S=F+\left[0, v_{i}\right]$. И очевидно, что (в кубильяже $\mathcal{Q})$ мы имеем соотношения $Q \prec S \prec R$. См. рисунок 10 ,

\section{0 Порядок на кубильяжах капсида}

Наиболее просто Предложение 7 работает для стандартного кубильяжа. Дело в том, что редукция $\widetilde{\mathcal{Q}}=\mathcal{Q}_{-n}$ стандартного кубильяжа $\mathcal{Q}=\mathcal{Q}_{s t}$ зонотопа $Z(n, d)$ по цвету $n$ является стандартным кубильяжем (зонотопа $Z(n-1, d)$ ). Более того, сжатие перегородки $\mathcal{P}=\mathcal{P}_{n}$ цвета $n$ в $\mathcal{Q}$ дает антистандартный кубильяж зонотопа 


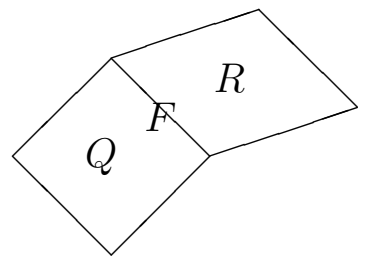

$\mathcal{Q}_{-i}$

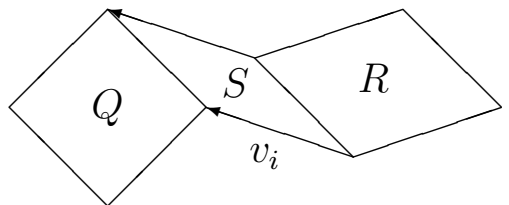

$\mathcal{Q}$

Рис. 10:

$Z^{\prime}=Z(n-1, d-1)$. Этими соображениями можно воспользоваться, чтобы полностью описать естественный порядок на стандартном кубильяже. Мы проделаем это упражнение для случая $n=d+1$, то есть для капсида.

Пусть $\mathcal{Q}$ - стандартный кубильяж капсида $Z=Z(d+1, d)$. Редуцированный кубильяж $\widetilde{\mathcal{Q}}$ - это тривиальный кубильяж куба $\widetilde{Z}=Z(d, d)$. Перегородка $\mathcal{P}$ (цвета $n=d+1$ ) примыкает к невидимой (задней) части $\partial_{-}(\widetilde{Z})$ границы куба $\widetilde{Z}$; более точно, она получается суммированием этой задней части границы с отрезком $\left[0, v_{n}\right]$. Поэтому кубы из перегородки имеют вид $F+\left[0, v_{n}\right]$, где $F$ пробегает невидивые фасеты куба $\widetilde{Z}$. (Посмотрите на рис. 6 и 7.) Видно, что все они $\succ$ куба $\widetilde{Z}$. И остается разобраться с порядком на кубах перегородки $\mathcal{P}$. Но, как мы знаем из Леммы о реверсе, этот порядок противоположен порядку кубильяжа, полученного проекцией $\pi$ (сжатием) задней мембраны $\partial_{+}(\widetilde{Z})$, то есть антистандартного кубильяжа 'меньшего' капсида $Z^{\prime}=Z(d, d-1)$. Так что порядок на кубах из $\mathcal{P}-$ это в точности порядок на стандартном кубильяже капсида $Z(d, d-1)$. Индукцией по $d$ мы получаем

Предложение 8 Естественный порядок на стандартном кубильяже капсида $Z(d+1, d)-$ полный (или линейный).

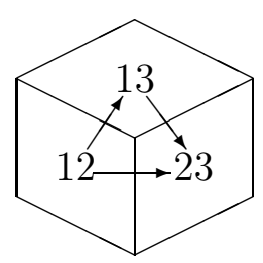

Рис. 11: Порядок на стандартном тайлинге зоногона Z(3,2).

Иначе говоря, это цепь из $n=d+1$ элемента. Более того, мы видим, что уже отношение $\prec$ в этом случае транзитивно. Куб $\widetilde{Z}$ - минимальный элемент этого порядка. Заметим, что его тип равен $[d]=[n]-n$. Та же индукция, что и выше, показывает, что в терминах типов кубов порядок на стандарном кубильяже капсида $Z(d+1, d)$ совпадает с лексикографическим порядком $\prec_{\text {lex }}$ на множестве $G r([d+1], d)$ подмножеств размера $d$ в $[d+1]$. Например, при $d=6$ этот порядок выглядит так:

$$
12345 \prec 12346 \prec 12356 \prec 12456 \prec 13456 \prec 23456 .
$$


Для антистандартного кубильяжа капсида порядок $\prec$ тоже линейный, но в терминах типов меняется на противоположный, антилексикографический, $\prec$ antilex .

Мы оставляем как упражнение разбор следующего по сложности случая: стандартного кубильяжа зонотопа $Z(d+2, d)$. Ответ для стандартного кубильяжа зонотопа $Z(6,4)$ изображен на рис. 123

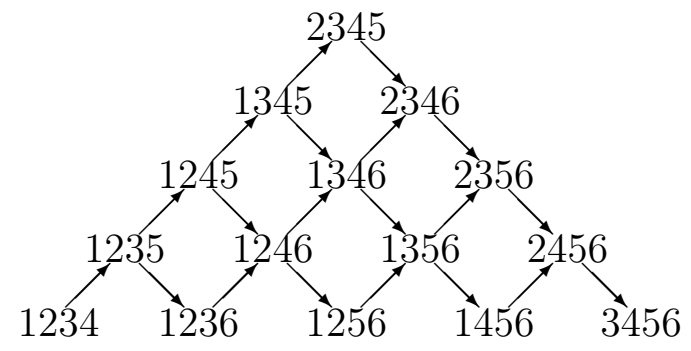

Рис. 12: Горка

При рассмотрении естественного порядка на общем кубильяже важную роль играет следующее соображение. Каждый капсид (то есть подзоногон $Z(d+1, d)$ в кубильяже $\mathcal{Q})$ задает свой тип $K$, подмножество размера $d+1$ в $[n]$. В свою очередь, пусть $K \subset[n]$ - произвольное подмножество размера $d+1$. Рассмотрим в $\mathcal{Q}$ множество $\mathcal{F}(K)$, состоящее из кубов $Q \in \mathcal{Q}$, типы которых содержатся в $K$. Число таких кубов равно $d+1$, и они параметризуются своими типами $K-i$, где $i$ пробегает $K$. В совокупности эти кубы представляют как бы 'разбежавшийся' капсид (настоящий капсид состоит из кубов, тесно прижатых друг к другу). Редуцируя цвета, не входящие в $K$, мы 'прижмем' эти кубы друг к другу. Более точно, пусть $\mathcal{Q}_{K}$ обозначает кубильяж, полученный из $\mathcal{Q}$ редукцией всех цветов, не принадлежащих $K$. Как множество, $\mathcal{Q}_{K}$ отождествляется с $\mathcal{F}(K)$. Кубильяж $\mathcal{Q}_{K}$ является кубильяжем капсида $Z(K, d)$ и поэтому либо стандартный, либо антистандартный. И так как (см. Пример 3 выше) естественный порядок $\preceq$ на $\mathcal{F}(K)$ совпадает с порядком на $\mathcal{Q}_{K}$, то он во-первых, линейный (то есть $\mathcal{F}(K)$ - это цепь для $\preceq$ ), и во-вторых, в терминах типов он либо лексикографический, либо антилексикографический. Причем какой - легко понять. В множестве $K$ как подмножестве $[n]$ есть максимальный элемент, пусть это $k$. Тогда все определяется тем - будет ли куб $Q$ с типом $K-k$ расположен раньше перегородки $\mathcal{P}_{k}$ цвета $k$ в $\mathcal{Q}$ (и тогда $Q$ - минимальный элемент в $\mathcal{F}(K)$, а порядок лексикографический), либо позже перегородки (и тогда куб $Q$ максимален, а порядок $\preceq$ на подмножестве $\mathcal{F}(K)$ антилексикографический).

Итак, ограничения $\preceq$ на такие 'капсидо-подобные' системы $\mathcal{F}(K)$ в $\mathcal{Q}$ являются линейными порядками (цепями). В свою очередь, эти цепи однозначно определяют $\preceq$. Более точно,

Предложение 9 Естественный порядок $\preceq$ на кубильяже $\mathcal{Q}$ - это транзитивное замыкание иепей $\left(\mathcal{F}(K), \preceq_{\mathcal{F}(K)}\right)$ по всем $K \subset[n]$ размера $d+1$.

\footnotetext{
${ }^{3}$ Отметим аналогию с рисунками колчанов в [24, Table 1].
} 
Для доказательства достаточно проверить, что если $Q \prec R$ для кубов $Q$ и $R$ кубильяжа $\mathcal{Q}$, то $Q$ и $R$ встечаются в некотором 'разбросанном' капсиде $\mathcal{F}(K)$. В самом деле, надо взять в качестве $K$ объединение типов $Q$ и $R$; так как эти кубы соседние, размер $K$ равен $d+1$.

Таким образом, естественный порядок $\preceq$ на кубильяже $\mathcal{Q}$ можно было бы определять не через отношение $\prec$ 'непосредственного следования', но через цепи $\mathcal{F}(K)$ размера $d+1$. Мы вернемся к этому обстоятельству в комбинаторной части.

\section{1 Стэки и мембраны}

Определение. Подмножество $\mathcal{S}$ кубов в $\mathcal{Q}$ называется стэком, если с каждым кубом $Q$ подмножество $\mathcal{S}$ содержит и меньшие (относительно $\prec$ или $\preceq$ ) кубы. Иными словами, это порядковый идеал в посете $(\mathcal{Q}, \preceq)$. Телом стэка $\mathcal{S}$ называется подмножество в $Z=Z(n, k)$, являющееся объединением кубов, входящих в $\mathcal{S}$, плюс множество точек видимой границы зонотопа $Z$.

Интерес стэков вызван тем, что они задают мембраны кубильяжа $\mathcal{Q}$. В самом деле, любая прямая, параллельная $d$-ой оси координат, проходя внутри зонотопа $Z$, сначала идет по телу стэка $\mathcal{S}$, потом в какой-то момент пересекает границу стэка и уже не возвращается в стэк. Множество этих 'последних' точек стэка образует мембрану $\mathcal{M}=\mathcal{M}(\mathcal{S})$ в кубильяже $\mathcal{Q}$. В самом деле, оно состоит из фасет кубильяжа $\mathcal{Q}$ и биективно проектируется на $Z^{\prime}=\pi(Z)$. Обратно, если $\mathcal{M}$ мембрана кубильяжа $\mathcal{Q}$, и мы соберем все кубы, расположенные до мембраны, мы получим стэк $\mathcal{S}(\mathcal{M})$. Очевидно, что эти операции взаимно обратны, и мы получаем

Предложение 10 Множество $\mathrm{S}(\mathcal{Q})$ стэков в кубильяже $\mathcal{Q}$ и множество мембран $\mathbf{M}(\mathcal{Q})$ естественно биективны.

Следствие. Зонотоп $Z(d+2, d)$ имеет $2(d+2)$ различных кубильяжей.

Доказательство. Согласно Предложению 2 из раздела 6, задать кубильяж зонотопа $Z(d+2, d)$ - это то же самое, что задать мембрану в некотором кубильяже капсида $Z(d+1, d)$. У последнего имеется всего два кубильяжа - стандартный и антистандартный. И на каждом из них естественный порядок является цепью (длины $d+1)$. Соответственно, имеется $d+2$ стэка, а в силу предыдущего предложения $-d+2$ мембраны.

Легко понять, что посет $\mathbf{Q}(d+2, d)$ состоит из двух ветвей кольца размера $2(d+2)$, как на рисунке 13 .

Мембраны в кубильяже $\mathcal{Q}$ естественно упорядочены: мы говорим, что мембрана $\mathcal{M}$ кубильяжа $\mathcal{Q}$ встречается ранъше, чем мембрана $\mathcal{M}^{\prime}$, если любая прямая, параллельная $e_{d}$, пересекает $\mathcal{M}$ не позже, чем $\mathcal{M}^{\prime}$. В терминах стэков это в точности отношение включения соответствующих подмножеств в $\mathcal{Q}$ ). Мы получаем отсюда следующие утверждения.

Предложение 11 а) Множество мембран $\mathbf{M}(\mathcal{Q})$ кубильяжа $\mathcal{Q}$ образует дистрибутивную решетку. 


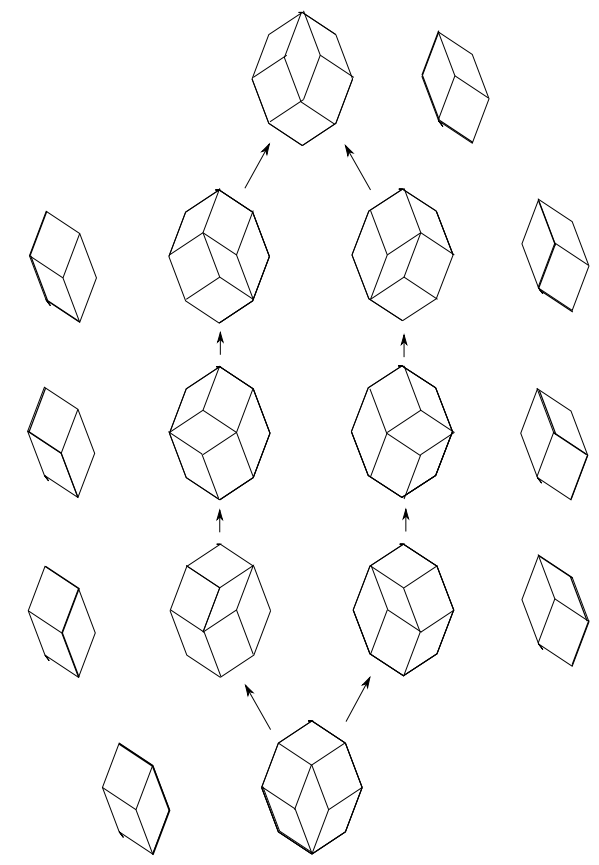

Рис. 13: "Кольцо". Изображены восемь кубильяжей зоногона $Z(4,2)$. Стрелки указывают повышающие флипы. Сбоку от каждого кубильяжа помещен кубильяж $Z(3,2)$ и мембрана в нем, задающие (см. Предложение 4) соответствующий кубильяж $Z(4,2)$.

b) Эта решетка градуирована (как и любая дистрибутивная, см. [18], 3.4); ранг мембраны $\mathcal{M}$ равен числу элементов в стэке $\mathcal{S}(\mathcal{M})$.

с) Для любой мембраны $\mathcal{M}$, если она не максимальна, существует куб $Q \in \mathcal{Q}$, который всеми своими видимыми фасетами входит в мембрану М. Добавление этого куба приводит к мембране $\mathcal{M}^{\prime}$, которая отличается от $\mathcal{M}$ повышающим флопом.

Таким образом, мы видим, что любую мембрану $\mathcal{M}$ в $\mathcal{Q}$ можно соединить с минимальной мембраной (видимой частью границы зонотопа) понижающими флопами (а с максимальной мембраной - повышающими флопами). А это означает также, что кубильяж $\pi(\mathcal{M})$ зонотопа $Z^{\prime}=Z(n, d-1)$ можно превратить в стандартный кубильяж зонотопа $Z^{\prime}$ серией понижающих флипов.

\section{2 Существование флипов}

Понятие флипа уже вводилось в разделе 8 как замена одного кубильяжа капсида $Z(d+1, d)$ в $Z$ другим кубильяжем. Здесь мы покажем существование флипов.

Теорема 1 Пусть $\mathcal{Q}$ - кубильяж зонотопа $Z=Z(n, k)$. Если кубильяж $\mathcal{Q}$ отличен от стандартного, то в нем можно сделать понижсающий флип.

Иными словами, если в кубильяже нельзя сделать понижающий флип, то он стандартный. Верно, конечно, и обратное: стандартный кубильяж не допускает 
понижающих флипов. Это видно, например, из того, что стандартный кубильяж реализуется видимой, или передней, мембраной в (любом) кубильяже зонотопа $\hat{Z}^{=} Z(n, d+1)$, и 'отступать назад' от такой мембраны уже некуда.

Доказательство. Рассмотрим перегородку $\mathcal{P}$ цвета $n$ в $\mathcal{Q}$. Если она проходит вплотную к невидимой стороне $Z$, мы просто 'выбросим' ее как из $\mathcal{Q}$, так и из $Z$, и получим кубильяж $\mathcal{Q}-\mathcal{P}$ зонотопа $Z(n-1, d)$. Если этот кубильяж $\mathcal{Q}-\mathcal{P}$ стандартный, тогда и $\mathcal{Q}$ - стандартный, вопреки предположению. Поэтому кубильяж $\mathcal{Q}-\mathcal{P}$ зонотопа $Z(n-1, d)$ не стандарный, и по индукции мы можем сделать в нем (а поэтому и в $Z$ ) понижающий флип.

Таким образом мы можем считать, что перегородка $\mathcal{P}$ не примыкает к невидимой части границы зонотопа $Z$, и за этой перегородкой реально есть кубы. Возьмем среди таких кубов (строго за перегородкой $\mathcal{P}$ ) минимальный куб $Q$ относительно естественного порядка $\preceq$ на $\mathcal{Q}$. Этот куб всей видимой стороной примыкает к перегородке $\mathcal{P}$. И если мы добавим к этому кубу $Q$ все кубы из перегородки $\mathcal{P}$, которые примыкают к $Q$, мы получим желаемый подзонотоп $Z_{0}$ (капсид). В самом деле, эти кубы устроены как суммы отрезка $\left[-v_{n}, 0\right]$ на видимые фасеты $Q$. И мы получаем подзонотоп типа $B n$, где $B$ - тип куба $Q$. Это как раз такой набор кубов, с которым можно делать понижающий флип, см. раздел 8.

В результате такого понижающего флипа с участием цвета $n$ мы передвигаем (отодвигаем) перегородку цвета $n$ 'на один куб' по направлению к невидимой границе зонотопа $Z$. Двигаясь так шаг за шагом, мы передвинем эту перегородку вплотную к невидимой границе $Z$. Однако мы могли бы сделать это сразу, за один 'большой' шаг. Для этого обозначим через $\mathcal{Q}_{+}$множество кубов нашего кубильяжа $\mathcal{Q}$, расположенных ЗА перегородкой $\mathcal{P}=\mathcal{P}_{n}$. После этого 'сдинем' весь этот набор кубов $\mathcal{Q}_{+}$на вектор $-v_{n}$. В результате это множество передвинется вплотную к передней (видимой) границе перегородки $\mathcal{P}$ и образует кубильяж зонотопа $Z(n-1, d)$. Если мы добавим к этому зонотопу произведение (сумму) его невидимой границы на отрезок $\left[0, v_{n}\right]$ (иными словами, сделаем эспансию этого кубильяжа вдоль 'задней' мембраны зонотопа $Z(n-1, k)$ по цвету $n)$, мы получим исходной зонотоп $Z$ и новый его кубильяж, в котором новая перегородка $\mathcal{P}^{\prime}$ цвета $n$ примыкает к невидимой стороне $Z$. Такую 'крупную' перестройку кубильяжа можно было бы назвать 'большим флипом', или обвалом, см. рис. 14.

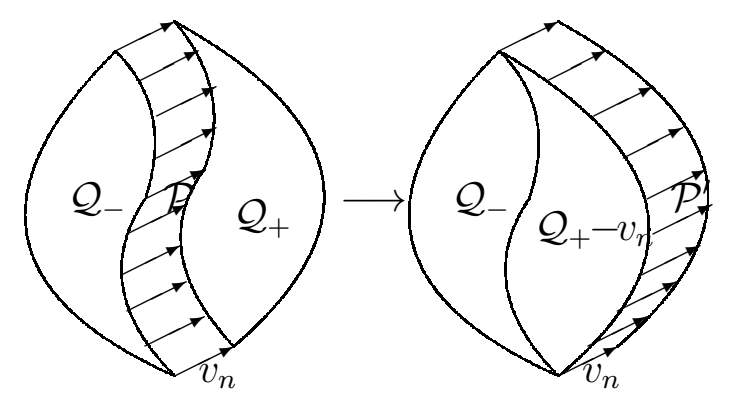

Рис. 14: обвал

В результате такого обвала мы получаем кубильяж $\widetilde{\mathcal{Q}}$ зонотопа $\widetilde{Z}=Z(n-1, d)$. 
С ним можно проделать такую же операцию подъема перегородки цвета $n-1$, и так далее. Эту последовательность из $n$ обвалов можно назвать стандартизацией кубильяжа $\mathcal{Q}$, потому что после каждого шага (обвала) кубильяж становится 'все более стандартным', а в конце процесса - полностью стандартным. Главное преимущество стандартизации - ее каноничность; если понижающие флипы мы могли делать в разных местах и в разной последовательности, то обвалы определены однозначно.

Симметрично можно делать 'антистандартизацию' кубильяжа, прижимая (на первом шаге) перегородку $\mathcal{P}$ к видимой границе зонотопа $Z(n, d)$ и продолжая в таком же духе; в результате через $n$ шагов мы получим антистандартный кубильяж.

Уже говорилось, как с помощью флипов вводится порядок $\leq$ на множестве $\mathrm{Q}(n, d)$ кубильяжей зонотопа $Z(n, d)$. А именно, мы полагаем $Q \leq Q^{\prime}$, если от $Q$ до $Q^{\prime}$ можно добраться с помощью последовательности повышающих флипов. Циклов при этом быть не может, так что это отношение $\leq$ действительно является отношением частичного порядка на множестве $\mathbf{Q}(n, d)$. Фактически, именно этот посет Манин и Шехтман называли высшим порядком Брюа и обозначали $B(n, d)$. Теорема 1 говорит, что этот посет обладает минимальным (стандартный кубильяж) и максимальным (антистандартный кубильяж) элементами. Подобные посеты часто оказываются решетками. И действительно, первый посет Брюа $B(n, 1)$ (т. н. слабый порядок Брюа) является решеткой. При малых $n$ посет $B(n, 2)$ тоже решетка (см. рисунок посета $B(5,2)$ в [19]), но уже $B(6,2)$ не является решеткой, [10]. Более того, этот посет в общем случае не есть посет вершин многогранника, [19]. И все же это градуированный (ранговый) посет.

\section{3 Мембраны в зонотопе}

До сих пор мы занимались мембранами в кубильяже. Теперь мы введем более общее понятие мембраны в зонотопе $Z=Z(n, k)$. Это снова $(d-1)$-мерный кубический комплекс $\mathcal{M}$, расположенный в $Z(n, k)$, причем выполнены два свойства (заимствованные у мембран в кубильяжах):

1) $\mathcal{M}$ (точнее, тело $\mathcal{M}$ ) проектируется биективно на $Z^{\prime}=Z(n, d-1)$,

2) ребра $M$ конгруэнтны векторам $v_{i}$ (впрочем, при $d>2$ это выводится из свойства 1) примерно как в Лемме 1).

Проекции кубов из $\mathcal{M}$ дают $(d-1)$-мерный кубильяж зонотопа $Z^{\prime}$, который мы снова обозначаем как $\mathcal{Q}^{\prime}=\pi(M)$. Обратно, если $\mathcal{Q}^{\prime}-$ кубильяж зонотопа $Z^{\prime}$, мы можем (единственным способом) построить мембрану $\mathcal{M}$, для которой $\left.\mathcal{Q}^{\prime}=\pi(\mathcal{M})\right)$. Делается это так. Для любой вершины $v^{\prime}$ кубильяжа $\mathcal{Q}^{\prime}$ мы задаем вершину $v$ как $\sum_{i \in s p\left(v^{\prime}\right)} v_{i}$. После этого организуем эти вершины в кубильяж, повторяя организацию в кубы вершин $v^{\prime}$ кубильяжа $\mathcal{Q}^{\prime}$. Или чуть иначе: мы строим мембрану $\mathcal{M}$ (точнее - тело этой мембраны) как некоторое естественное (кусочно линейное) сечение зонотопа $Z^{\prime}$ (сечение относительно проекции $\pi$ ). Как уже говорилось, каждая точки кубильяжа $\mathcal{Q}^{\prime}$ однозначно записывается через вектора $v_{i}^{\prime}$; остается переписать это через $v_{i}$. Тем самым, 
Множество $\mathbf{M}(n, d)$ мембран в зонотопе $Z=Z(n, d)$ отождествляется $c$ множеством $\mathbf{Q}(n, d-1)$ кубильяжей зонотопа $Z(n, d-1)$.

Поясним на примере $d=3$. Кубильяж зоногона $Z^{\prime}=Z(n, 2)$ - это ромбической тайлинг, чисто двумерная фигура. Переход к мембране в $Z=Z(n, 3)$ делает ее более рельефной: это как переход от плоского чертежа к трехмерному макету. Флипы, выглядящие как искусственные перетасовки ромбов, превращаются в более наглядные изгибания пленок-мембран. Однако пока наши мембраны 'висят в воздухе'. Следующее утверждение исправляет этот дефект.

Теорема 2 Для любой мембрань $\mathcal{M}$ зонотопа $Z=Z(n, d)$ существует кубильяю $\mathcal{Q}$ этого зонотопа $Z$, для которого она является мембраной.

Иными словами, мембрана $\mathcal{M}$ вписывается в некоторый кубильяж $\mathcal{Q}$.

Доказательство. Обозначим через $Z_{-}(\mathcal{M})$ область в $Z$ перед мембраной $\mathcal{M}$. Воспользуемся Теоремой 1. Делая с мембраной $\mathcal{M}$ понижающие флопы (точнее, мы делаем флипы с кубильяжем $\pi(\mathcal{M})$, а потом поднимаем их на мембраны), мы получаем серию мембран, идущую от $\mathcal{M}$ до 'передней' мембраны, то есть до видимой части границы $Z$. Тем самым мы получаем кубильяж области $Z_{-}(\mathcal{M})$.

Аналогично можно поступить с областью $Z_{+}(\mathcal{M})$ за мембраной; вместе они дадут кубильяж: в который вписана мембрана $\mathcal{M}$.

Более того, если делать каноническую стандартизацию кубильяжа $\pi(\mathcal{M})$, мы получим канонический (или 'стандартный') кубильяж области $Z_{-}(\mathcal{M})$ в зоногоне Z. 'Стандартность' этого кубильяжа проявляется в том, что в нем нельзя сделать понижающий флип.
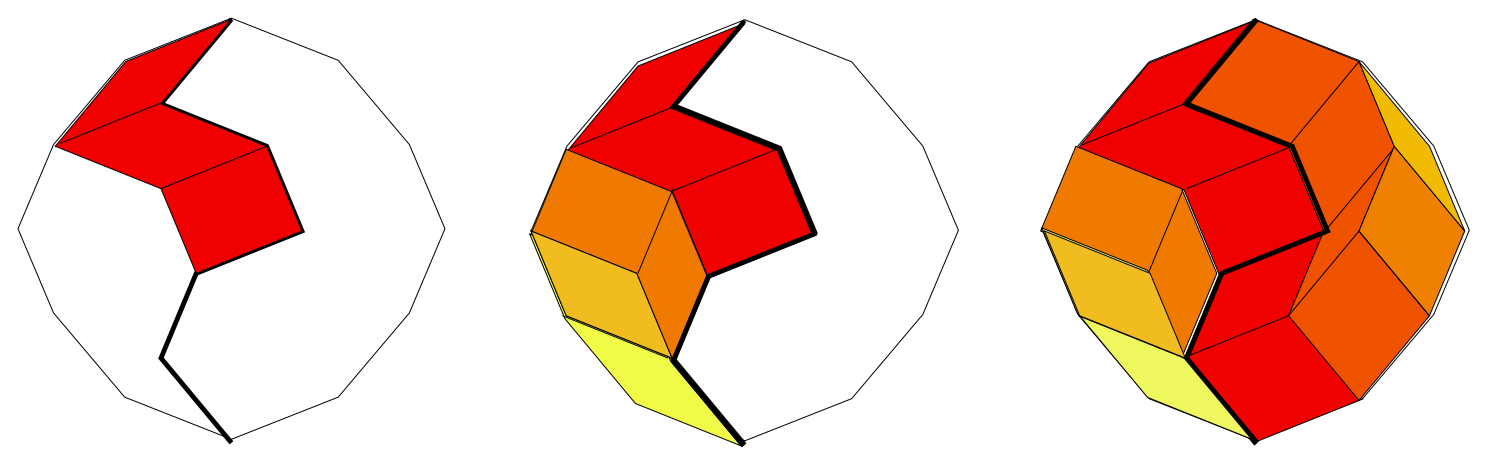

Рис. 15: Стадии канонизации. Дан ромбический тайлинг и мембрана-змейка $\mathcal{M}$ в нем. На рис. слева изображен первый шаг стандартизации области перед мембраной - построение части перегородки цвета 6. На рис. в центре уже завершено построение всех перегородок в области перед мембраной, то есть показана стандартизация этой области. На рис. справа показана (анти)стандартизация области за мембраной.

Симметрично можно поступить с областью $Z_{+}(\mathcal{M})$, расположенной в $Z$ после мембраны $\mathcal{M}$. Мы получим каноническое ('антистандартное') заполнение этой 
области. Объединяя эти два кубильяжа, мы получаем кубильяж $\mathcal{Q}$ уже всего зонотопа $Z$, в котором $\mathcal{M}$ является мембраной. Мы называем этот стандартноантистандартный кубильяж зонотопа $Z$ каноническим расширением мембраны $\mathcal{M}$ (или каноническим подгемом кубильяжа $\pi(\mathcal{M})$ ).

Замечание. Рассуждая как при доказательстве Теоремы 1, можно показать, что если кубильяж области $Z_{-}(\mathcal{M})$ отличен от стандартного (см. выше), то в нем можно сделать понижающий флип. Это показывает, что кубильяжи области $Z_{-}(\mathcal{M})$ (как и кубильяжи всего зонотопа) связаны флипами. Аналогичное замечание верно и для кубильяж области $Z_{+}(\mathcal{M})$.

Мембраны служат как бы средством, связывающим кубильяжи соседних размерностей. Кубильяж $\mathcal{Q}$ представляется 'сетью' мембран, а тем самым 'сетью' кубильяжей на 1 меньшей размерности. Напомним, что $\mathbf{Q}(n, d)$ - это множество кубильяжей зонотопа $Z(n, d)$. Скажем, что кубильяжи $\mathcal{Q}$ из $\mathbf{Q}(n, d)$ и $\mathcal{Q}^{\prime}$ из $\mathbf{Q}(n, d-1)$ согласованы, если $Q^{\prime}$ поднимается до мембраны $\mathcal{M}$ в $\mathcal{Q}$ (так что $\mathcal{Q}^{\prime}=\pi(\mathcal{M})$ ). Отношение согласованности можно рассматривать как соответствие $c$ из $\mathbf{Q}(n, d)$ в $\mathbf{Q}(n, d-1)$. Описанный выше канонический подъем кубильяжей задает сечение can $: \mathbf{Q}(n, d-1) \rightarrow \mathbf{Q}(n, d)$ этого соответствия $c$.

Обилие связей между кубильяжами побуждает взглянуть на них с категорной точки зрения. Мы поговорим об этом в Дополнении 1 про поликатегории.

\section{ЧАСТЬ ВТОРАЯ, КОМБИНАТОРНАЯ}

Под комбинаторным подходом к кубильяжам мы понимаем изучение систем подмножеств базисного множества $[n]$, генерируемых кубильяжами. Мы уже встречались с этим, когда рассматривали типы кубов кубильяжа. Однако если брать только типы кубов, то любой кубильяж дает все множество-грассманиан $\left(\begin{array}{c}{[n]} \\ d\end{array}\right)=$ $G r([n], d)$. Чтобы отразить специфику конкретного кубильяжа $\mathcal{Q}$, нужно перенести на $G r([n], d)$ естественный порядок $\preceq \mathcal{Q}$. Это перебрасывает мост между геометрическим подходом и подходом, который использовали Манин с Шехтманом [8, 14] и Циглер [10]. Напомним, что Манин и Шехтман, создатели высших порядков Брюа, работали именно в терминах порядков на $G r([n], d)$.

Другой выход в комбинаторику открывает понятие спектра. Если мы соберем в одну систему $S p(\mathcal{Q})$ спектры всех вершин кубильяжа $\mathcal{Q}$, то получим интересную систему подмножеств в $[n]$. Главное свойство таких систем - свойство разделенности, впервые обнаруженное Леклерком и Зелевинским [6] в случае $d=2$ и обобщенное и исследованное затем Галашиним и Постниковым [11, 12].

В процессе работы нам постоянно придется иметь дело с подмножествами и системами подмножеств в $[n]$. Само базисное множество $[n]$ можно понимать как аналог $n$-мерного векторного пространства (над произвольным полем, или над 'полем' $\mathbf{F}_{1}$ из одного элемента). С этой точки зрения подмножество в $[n]$ размера $k$ надо понимать как аналог векторного подпространства размерности $k$. Из-за этого множество всех подмножеств размера $k$ мы называем 'дискретным грассманианом' и обозначаем $\operatorname{Gr}([n], k)$ (вместо обычного обозначения $\left.\left(\begin{array}{c}{[n]} \\ k\end{array}\right)\right)$. Аналогом полного 
флага подпространств служит неуплотняемая цепь подмножеств в $[n]$, то есть фактически линейный порядок на $[n]$. Напомним, что мы рассматриваем $[n]$ как множество (цепь) с естественным порядком $(1<2<\ldots<n)$, что означает, что мы как бы фиксируем некоторый полный флаг.

Для удобства идентификации элементы базисного множества $[n]$ мы называем иветами и используем обычно буквы $i, j$ и т.д.; подмножества в $[n]$ обозначаются буквами $X, Y$ и т.д. и называются часто просто множествами. Для краткости множество вида $X \cup\{i\}$ обозначается обычно как $X i$. Подмножества в $2^{[n]}$ называются системами множеств и обозначаются рукописными буквами (типа $\mathcal{S}$ или $\mathcal{X}$ ).

\section{4 Допустимые порядки}

Если $Q$ - куб некоторого кубильяжа $\mathcal{Q}$ зонотопа $Z(n, d)$, то он определяет (и определяется) двумя множествами: спектром нижней (корневой) вершины $b(Q)$, $s p(b(Q)) \subset[n]$, и типом $\tau(Q) \subset[n]$. Эти два подмножества не пересекаются, и второе имеет размер $d$. Фактически это уже упоминавшаяся кодировка знаковыми векторами; $Q \mapsto \frac{\tau(Q)}{s p(b(Q))}$. Согласно Предложению 1, отображение типа $\tau$ задает биекцию множества $\mathcal{Q}$ всех кубов кубильяжа $\mathcal{Q}$ с грассманианом $G r([n], d)$. Если перенести с помощью этой биекции $\tau$ естественный порядок $\preceq$ на $\mathcal{Q}$ (см. раздел $9)$, мы получим некоторый порядок $\preceq_{\mathcal{Q}}$ на грассманиане $\operatorname{Gr}([n], d)$.

\section{Предложение 12 Порядок $\preceq_{\mathcal{Q}}$ определяет кубильяљ $\mathcal{Q}$.}

Иначе говоря, он позволяет однозначно восстановить кубильяж.

Доказательство. Пусть есть два кубильяжа $\mathcal{Q}_{1}$ и $\mathcal{Q}_{2}$ зонотопа $Z=Z(n, d)$ с одним и тем же порядком на $G r([n], d)$. И пусть $\mathcal{S}$ - (порядковый) идеал в $G r([n], d)$; покажем индукцией по размеру $\mathcal{S}$, что кубы (в $\mathcal{Q}_{1}$ и $\left.\mathcal{Q}_{2}\right)$, соответствующие $\mathcal{S}$ (то есть $\left.\tau^{-1}(\mathcal{S})\right)$, расположены в $Z$ одинаково. Пусть $K \in G r([n], d)$ - максимальный элемент в $\mathcal{S}$, и $\mathcal{S}^{\prime}=\mathcal{S}-\{K\}$. По индуктивному предположению кубы, соотвествующие $\mathcal{S}^{\prime}$, расположены в $Z$ одинаково. И лежат в 'передней' части относительно некоторой мембраны $\mathcal{M}^{\prime}$. Тогда куб $Q_{1}$ (в $\mathcal{Q}_{1}$ с типом $\left.K\right)$ примыкает всей своей видимой границей к определенному участку (капсиду) этой мембраны $\mathcal{M}^{\prime}$, точно так же, как и куб $Q_{2}$ (из $\mathcal{Q}_{2}$ с типом $K$ ). То есть они примыкают к одному и тому же участку $\mathcal{M}^{\prime}$ и потому совпадают.

Можно пояснить это и чуть иначе. Пусть $Q_{1}$ (соотв., $\left.Q_{2}\right)$ - кубы в кубильяжах $\mathcal{Q}_{1}$ (соотв., $\left.\mathcal{Q}_{2}\right)$ с одним и тем же типом (скажем, $\left.K\right)$. И пусть $b_{1}=b\left(Q_{1}\right)$ и $b_{2}=$ $b\left(Q_{2}\right)$ - их корневые вершины. Достаточно показать, что $b_{1}=b_{2}$. Возьмем какуюнибудь видимую фасету $F_{1}$ куба $Q_{1}$ (с типом $K-i$ для некоторого $i \in K$ ), и рассмотрим часть тоннеля типа $K-i$, идущую от $F_{1}$ и передней границе зонотопа $Z$. Мы не знаем, как проходит этот тоннель, но мы знаем, что он составлен из кубов $Q$, которые а) лежат в идеале $\mathcal{S}$, и b) содержат $K-i$ в своем типе $\tau(Q)$. Этот тоннель выходит к передней части границы $Z$ по единственной фасете $\widetilde{F}$ типа $K-i$. Обозначим его корневую вершину как $\widetilde{b}$. Тогда мы получаем, что $b_{1}=\widetilde{b}+\sum_{j}( \pm) v_{j}$, где сумма берется по тем $j$, что $K-i+j$ принадлежит $\mathcal{S}$. Знаки 
же определяются как знаки определителя $\operatorname{det}\left(\ldots v_{k} \ldots v_{j}\right)$, где $k$ пробегает $K-i$. Так как это выражение зависит от $K-i$ и от $\mathcal{S}$, но не от $\mathcal{Q}$, то $b_{2}=b_{1}$.

Полученное утверждение поднимает вопрос о том, какими условиями выделяются порядки вида $\preceq_{\mathcal{Q}}$. Одно такое условие подсказывает Предложение 9 раздела 10. Назовем родителем подмножество $K$ в $[n]$ размера $d+1$, а его семъей - систему $\mathcal{F}(K)$ подмножеств размера $d$ в $K$. (Иначе говоря, это образ $G r(K, d)$ в $G r([n], d)$ при естественном вложении. Манин и Шехтман называли это naкeтом.) У семьи есть два выделенных линейных порядка, а именно, лексикографический $\preceq_{\text {lex }}$ и противоположный к нему антилексикографический $\preceq_{\text {alex }}$. Если $K=\left\{i_{1}<i_{2}<\ldots<i_{d+1}\right\}$, то лексикографический порядок $\preceq_{\text {lex }}$ на $\mathcal{M}(k)$ имеет вид

$$
K-i_{d+1}<K-i_{d}<\ldots<K-i_{1},
$$

а антилексикографический - противоположный.

Определение. Частичный порядок $\preceq$ на $G r([n], d)$ называется допустимым, если его ограничение на любую семью $\mathcal{F}(K)$ (где $K$ - произвольное множество размера $d+1)$ есть лексикографический или антилексикографический порядок.

Разумеется, любое усиление допустимого порядка также допустимое. В частности, любое линейное расширение. Манин и Шехтман работали именно с линейными допустимыми порядками.

В этих терминах Предложение 9 утверждает, что порядок на $G r([n], d)$, индуцированный некоторым кубильяжем, допустим. Оказывается, это условие не только необходимое, но и в каком-то смысле достаточное.

Теорема 3 Пусть $\preceq$ - допустимый порядок на $G r([n], d)$. Тогда существует (u притом единственныц, см. Предложение 12) кубилъяж $\mathcal{Q}$, такой что $\preceq-$ - усиление $\preceq_{\mathcal{Q}}$.

Чтобы сделать идею доказательства более понятной, представим, что порядок $\preceq$ уже происходит из кубильяжа $\mathcal{Q}$. Пусть $\mathcal{P}$ - перегородка цвета $n$; произведем редукцию цвета $n$. В результате перегородка сожмется на мембрану $\mathcal{M}$, а кубильяж $\mathcal{Q}$ редуцируется в кубильяж $\mathcal{Q}^{\prime}=\mathcal{Q}_{-n}$ зонотопа $Z^{\prime}=Z(n-1, d)$. $\mathcal{Q}^{\prime}$ как множество кубов естественно вкладывается в $\mathcal{Q}$. И ограничение естественного порядка кубильяжа $\mathcal{Q}$ на $\mathcal{Q}^{\prime}$ сильнее естественного порядка на $\mathcal{Q}^{\prime}$ (Предложение 7 из раздела 9$)$. Так что ограничение $\preceq$ на $G r([n-1], d)$ - допустимый порядок, и по индукции мы можем восстановить $\mathcal{Q}^{\prime}$. Остается восстановить еще и мембрану $\mathcal{M}$. Но она задается множеством (типов) кубов, расположенных в $\mathcal{Q}^{\prime}$ до мембраны. Или, что то же самое, множеством кубов, расположенных в $\mathcal{Q}$ до перегородки. Пусть $Q$ - такой куб, и $T \subset[n-1]$ - его тип. Рассмотрим родителя $K=T n$ и его семью как набор кубов в $\mathcal{Q}$. Эта семья 'начинается' с $Q$ и затем идут кубы из перегородки $\mathcal{P}$, так как их типы содержат цвет $n$. Из этого следует, что $Q-$ минимальный член этой семьи. Напротив, если куб $Q$ взят за перегородкой, то $Q$ - максимальный член семьи. Таким образом, кубы (в $\mathcal{Q}^{\prime}$ ) до мембраны $\mathcal{M}$ - это те кубы, тип которых $T$ минимален в семье с родителем $T n$. (Все это пояснение можно рассматривать как третье доказательство Предложения 12.) 
Теперь перейдем к доказательству, которое представляет собой обращение предыдущих аргументов. Мы начинаем с допустимого порядка $\preceq$ на $G r([n], d)$. Ограничиваем его на $G r([n-1], d) \subset G r([n], d)$; очевидно, что это снова допустимый порядок на $G r([n-1], d)$. По индукции он происходит из некоторого кубильяжа $\mathcal{Q}^{\prime}$ зонотопа $Z^{\prime}=Z(n-1, d)$. Теперь образуем подмножество $\mathcal{T}$ в $\operatorname{Gr}([n-1], d)$, состоящее из таких $T$, что ограничение $\preceq$ на семью $\mathcal{F}(T n)$ родителя $T n$ - лексикографический порядок.

Мы утверждаем, что $\mathcal{T}$ - идеал относительно естественного порядка $\varliminf^{\prime}$ на $\mathcal{Q}^{\prime}$. В самом деле, пусть $Q \in \mathcal{T}$ и $R \prec Q$, то есть $R$ непосредственно предшестует кубу $Q$ в кубильяже $\mathcal{Q}^{\prime}$ (см. раздел 9 ). Нужно показать, что $R$ тоже принадлежит $\mathcal{T}$. Предположим противное, что ограничение $\preceq$ на семью родителя $\tau(R) n-$ антилексикографический порядок. В частности, $\tau(\bar{R})$ - максимальный элемент в семье $\mathcal{F}(\tau(R) n)$. В свою очередь, $\tau(Q)$ - минимальный элемент в семье $\mathcal{F}(\tau(Q) n)$. Кубы $R$ и $Q$ имеют общую фасету $F$, так что тип $F, \tau(F)$, содержится как в $\tau(R)$, так и в $\tau(Q)$. Поэтому множество $\tau(F) n$ лежит как в семье $\mathcal{F}(\tau(R) n)$, так и в семье $\mathcal{F}(\tau(Q) n)$. Отсюда следует, что $\tau(Q) \preceq \tau(F) n \preceq \tau(R)$ и по транзитивности $\tau(Q) \preceq \tau(R)$. Но это противоречит тому, что $R \prec Q$ и, следовательно, $\tau(R) \prec \tau(Q)$.

Итак, $\mathcal{T}$ - идеал в $\mathcal{Q}^{\prime}$. Этот идеал определяет мембрану $\mathcal{M}$ в $\mathcal{Q}^{\prime}$; делая экспансию цвета $n$ в этой мембране, мы получаем кубильяж $\mathcal{Q}$ (см. Предложение 4 раздела 6 ). Остается убедиться, что исходное отношение $\preceq$ сильнее, чем отношение $\preceq_{\mathcal{Q}}$. То есть если куб $R$ непосредственно предшествовал кубу $S$ в кубильяже $\mathcal{Q}, R \prec S$, то $\tau(R) \preceq \tau(S)$.

Это очевидно, если $R$ и $S$ лежат в $\mathcal{Q}^{\prime}$. Это почти очевидно, если один из кубов лежит в перегородке $\mathcal{P}$ (полученной экспансией мембраны $\mathcal{M}$ ), а другой - нет. Пусть, к примеру, $S$ лежит в перегородке, а $R$ - нет. Тогда куб $R$ расположен до перегородки. По построению идеала $\mathcal{T}$ семья $\mathcal{F}(\tau(R) \cup \tau(S))$ 'лексикографическая', и $\tau(R)$ - минимальный член этой семьи, откуда $\tau(R) \preceq \tau(S)$.

Осталось рассмотреть случай, когда $R$ и $S$ лежат в перегородке. Делая редукцию всех цветов, не вошедших в $\tau(R) \cup \tau(S)$, мы можем считать, что наш кубильяж $\mathcal{Q}$ - один из двух кубильяжей капсида, где утверждение очевидно в силу результатов раздела 10. Это завершает доказательство теоремы 3.

Теорема 3 перебрасывает мостик между геометрическим подходом (кубильяжами) и подходом Манина-Шехтмана, основанным на рассмотрении допустимых порядков на грассманианах $G r([n], d)$. Существование такой связи было анонсировано в работе Воеводского и Капранова [9] и более обстоятельно изложено в [20, Theorem 2.1].

Некоторое неудобство описания кубильяжей через допустимые порядки состоит в том, что разные порядки могут приводить к одному кубильяжу. Мы уже отмечали, что если $\preceq$ - допустимый порядок, то любое его усиление $\preceq$ тоже допустимый порядок и задает тот же кубильяж, что и $\preceq$. Чтобы восстановить однозначность, можно работать с минимальными допустимыми порядками. Конечно, это в точности порядки, порожденные (в смысле транзитивного замыкания) ограничениями на всевозможные семьи в $G r([n], d)$. Такие минимальные допустимые порядки задаются указанием для каждого родителя (множества $K$ размера $d+1)$, лексикографически или антилексикографически упорядочена семья $\mathcal{F}(K)$ этого 
родителя. Иначе говоря, отображением $\sigma: G r([n], d+1) \rightarrow\{+,-\}$, где + соответствует лексикографии. Конечно, это назначение $\sigma$ не может быть произвольным; надо, чтобы в результате получалось ацикличное отношение (ср. с Предложением 6). Вопрос о том, какие назначения дают ацикличность (и, следовательно, приводят к кубильяжам) мы обсудим в следующем разделе.

\section{5 Инверсии}

Раздел 13 позволяет рассматривать кубильяжи зонотопа $Z(n, d-1)$ как мембраны в зонотопе $Z(n, d)$ на единицу большей размерности. Это открывает еще один способ задания кубильяжей.

Пусть $\mathcal{Q}^{\prime}$ - кубильяж зонотопа $Z^{\prime}=Z(n, d-1)$. Реализуем его как мембрану $\mathcal{M}=\mathcal{M}\left(\mathcal{Q}^{\prime}\right)$ в зонотопе $Z=Z(n, d)$. Согласно Теореме 2, существует (и как правило, не один) кубильяж $\mathcal{Q}$ зонотопа $Z$, в который вписана мембрана $\mathcal{M}$. Эта мембрана делит кубы кубильяжа $\mathcal{Q}$ на расположенные до $\mathcal{M}$ и после $\mathcal{M}$. Кубы, расположенные до мембраны $\mathcal{M}$, соберем в множество $\mathcal{Q}_{-}(\mathcal{M})$. Это множество зависит, конечно, от кубильяжа $\mathcal{Q}$, содержащего мембрану $\mathcal{M}$. Однако множество типов кубов из $\mathcal{Q}_{-}(\mathcal{M})$ уже зависит только от мембраны $\mathcal{M}$, но не от $\mathcal{Q}$. Это видно из следующей леммы.

Лемма 3 Пусть $Q$ - куб кубильяља $\mathcal{Q}$, расположенный до (соотв., после) мембрань $\mathcal{M}, и K=\left\{k_{1}<k_{2}<\ldots<k_{d}\right\}$ - тип куба $Q$. Тогда существует вершина $v$ мембраны $\mathcal{M}$, такая что $K \cap s p(v)=\left\{k_{d}, k_{d-2}, \ldots\right\}$ (соотв., $K \cap s p(v)=$ $\left.\left\{k_{d-1}, k_{d-3}, \ldots\right\}\right)$.

Доказательство. Произведем редукцию всех цветов, отличных от цветов $K$. В результвте мы получим зонотоп $Z^{\prime}=Z(K, d)$ (состоящий из единственного куба $\left.Q=Z^{\prime}\right)$ и мембрану $\mathcal{M}^{\prime}$ в $Z^{\prime}$. Пусть $Q$ располагался до мембраны $\mathcal{M}$; тогда куб $Q=$ $Z^{\prime}$ тоже располагается до мембраны $\mathcal{M}^{\prime}$, то есть мембрана $\mathcal{M}^{\prime}$ - это невидимая часть границы $Q$. Пусть теперь $v^{\prime}$ - это 'голова' $h(Q)$ куба $Q$ (см. раздел 7$)$; как мы знаем, ее спектр равен $\left\{k_{d}, k_{d-2}, \ldots\right\}$. Пусть $v$ - 'прообраз' $v^{\prime}$ в мембране $\mathcal{M}$, то есть та вершина мембраны $\mathcal{M}$, которая редуцировалась в $v^{\prime}$. Ясно, что $s p(v)$ отличается от $s p\left(v^{\prime}\right)$ только цветами, не входящими в $K$, откуда $s p(v) \cap K=\left\{k_{d}, k_{d-2}, \ldots\right\}$.

Аналогично рассуждаем в случае, когда $K$ располагается после мембраны.

Замечание. Менее техническое рассуждение такое. Кубильяжи области в зонотопе $Z(n, d)$, расположенной перед мембраной $\mathcal{M}$, могут быть получены друг из друга с помощью флипов (см. замечание из раздела 13). А флипы не меняют типы кубов, но лишь переставляют их.

Пусть $\mathcal{Q}$ - кубильяж зонотопа $Z(n, d), \mathcal{M}$ - соответствующая мембрана в зонотопе $Z(n, d+1)$ (так что $\mathcal{Q}=\pi(\mathcal{M})$ ), а $\mathcal{Q}^{\prime}$ - некоторый кубильяж $Z(n, d+1)$, содержащий мембрану $\mathcal{M}$ (такой существует по Теореме 2 ).

Определение. Системой инверсий $\operatorname{Inv}(\mathcal{Q})$ кубильяжа $\mathcal{Q}$ называется система $\tau\left(\mathcal{Q}_{-}^{\prime}(\mathcal{M})\right)$ в $G r([n], d+1)$ (независящая от выбора $\mathcal{Q}^{\prime}$ в силу Леммы 3). Система $\operatorname{Inv}(\mathcal{Q})$ обозначается также как $\operatorname{Inv}(\mathcal{M})$. 
То же рассуждение, как при доказательстве Леммы 3 (с редукцией всех цветов, не входящих в тип куба $Q$ ), дает еще одно описание системы инверсий:

Предложение 13 Родитель $T \in G r([n], d+1)$ принадлежит $\operatorname{Inv}(\mathcal{Q})$ тогда $u$ только тогда, когда семъя этого фатера в $G r([n], d)$ упорядочена отношением $\preceq_{\mathcal{Q}}$ антилексикографически.

Или

$\operatorname{Inv}(\mathcal{Q})=\left\{T \in G r([n], d+1)\right.$, ограничение $\preceq_{\mathcal{Q}}$ на $G r(T, d)$ - антилексикография $\}$.

Например, система Inv для стандартного кубильяжа пустая. Напротив, Inv для антистандартного кубильяжа - все $G r([n], d)$. Когда мы делаем повышающий флип в кубильяже $\mathcal{Q}$, мы добавляем к домембранным кубам еще один куб. Так что при этом система $\operatorname{Inv}(\mathcal{Q})$ увеличивается на один элемент.

Важное свойство системы инверсий $\operatorname{Inv}(\mathcal{Q})$ в том, что она позволяет однозначно восстановить кубильяж $\mathcal{Q}$.

Предложение 14 Отображение $\operatorname{Inv}: \boldsymbol{Q}(n, d) \rightarrow 2^{G r([n], d+1)}$ инбективно.

Доказательство. Из Предложения 13 следует, что порядок $\preceq_{\mathcal{Q}}$ определяет $\mathcal{Q}$. Но порядок $\preceq_{\mathcal{Q}}$ порождается порядками на семьях, а система $\operatorname{Inv}(\mathcal{Q})$ как раз и показывает, какая семья упорядочена антилексикографически.

Как в предыдущем разделе, это поднимает вопрос о том, какие системы в $G r([n], d)$ являются 'инверсными', то есть происходят (с помощью Inv) из кубильяжей $Z(n, d-1)$. Вопрос был решен Циглером в статье [10] (хотя фундамент для решения был заложен в более ранней работе Лас Вернаса [21]). Введем следующее

Определение. Множество $\mathcal{S}$ в $\operatorname{Gr}([n], d)$ называется консистентным, если для любого $T \subset[n]$ размера $d+1$ семья $\mathcal{F}(T)$ этого родителя пересекается с $\mathcal{S}$ по начальному или конечному отрезку $\mathcal{F}(T)$ (семья $\mathcal{F}(T)$ располагается в лексикографическом порядке).

Лемма 4 Для любой мембранъ $\mathcal{M}$ в $Z(n, d)$ система $\operatorname{Inv}(\mathcal{M})$ образует консистентное подмножество в $G r([n], d)$.

Доказательство. Включим мембрану $\mathcal{M}$ в кубильяж $\mathcal{Q}$ зонотопа $Z=Z(n, d)$, и пусть $\preceq$ - естественный порядок на $\mathcal{Q}$, а $\preceq_{\mathcal{Q}}$ - соответствующий (допустимый) порядок на грассманиане $G r([n], d)$. Мы знаем из раздела 11 , что $\operatorname{Inv}(\mathcal{M})$ - это идеал относительно $\preceq_{\mathcal{Q}}$. С другой стороны, для любой семьи $\mathcal{F}(T)$ порядок $\preceq_{\mathcal{Q}}$ индуцирует лексикографию или антилексикографию. В первом случае пересечение $\operatorname{Inv}(\mathcal{M})$ с $\mathcal{F}(T)$ будет идеалом относительно лексикографического порядка, то есть начальным отрезком. Во втором - идеалом относительно противоположного порядка, то есть конечным отрезком.

Теорема 4 Обратно, пусть $\mathcal{S}$ - консистентная система в $G r([n], d)$. Тогда существует мембрана $\mathcal{M}$ в зонотопе $Z=Z(n, d)$, для которой $\mathcal{S}=\operatorname{Inv}(\mathcal{M})$. 
Мембрана $\mathcal{M}$ единственна, согласно Предложению 14.

Доказательство. Рассмотрим подсистему $\mathcal{S}^{\prime}$ в $\mathcal{S}$, состоящую из множеств $S \in \mathcal{S}$, не содержащих цвет $n$. Система $\mathcal{S}^{\prime}$ лежит в $G r([n-1], d)$ и тоже консистентна. Поэтому по индукции $\mathcal{S}^{\prime}$ реализуется некоторой мембраной $\mathcal{M}^{\prime}$ в $Z^{\prime}=Z([n-$ $1], d)$, так что $\mathcal{S}^{\prime}=\operatorname{Inv}\left(\mathcal{M}^{\prime}\right)$. Дополним эту мембрану до кубильяжа $\mathcal{Q}^{\prime}$ зонотопа $Z^{\prime}$ (Теорема 2). И затем сделаем экспансию по цвету $n$ мембраны $\mathcal{M}^{\prime}$ в $\mathcal{Q}^{\prime}$. Мы получаем кубильяж $\mathcal{Q}$ зонотопа $Z$, редукция которого $\mathcal{Q}_{-n}$ равна $\mathcal{Q}^{\prime}$; в частности, мембрана $\mathcal{M}^{\prime}$ 'утолщается' до перегородки $\mathcal{P}$ в $\mathcal{Q}$ цвета $n$. Кубы кубильяжа $\mathcal{Q}$, типы которых принадлежат $\mathcal{S}-\mathcal{S}^{\prime}$, лежат в $\mathcal{P}$.

Мы утверждаем, что $\mathcal{S}$ является стэком (идеалом) в кубилъяжсе $\mathcal{Q}$; в этом случае $\mathcal{M}$ - это мембрана, соответствующая стэку $\mathcal{S}$. Для этого нужно проверить, что если $R \prec S$ - два куба из перегородки $\mathcal{P}$, и $S$ принадлежит $\mathcal{S}$, то и $R$ принадлежит $\mathcal{S}$. Но кубы $R$ и $S$ соседние, поэтому объединение их типов, то есть $T=\tau(R) \cup \tau(S)$ имеет размер $d+1$. Рассмотрим семью $\mathcal{F}$ этого родителя $T$, расположенную лексикографически. Минимальным элеменом ('начальным членом') этой семьи служит множество $T-n$; пусть $Q-$ куб в $\mathcal{Q}$ с типом $T-n$. Этот куб не принадлежит перегородке $\mathcal{P}$, поэтому он лежит либо до перегородки, либо после неe.

Рассмотрим случай, когда $Q$ лежит до перегородки. Тогда в естественном порядке $\preceq$ на $\mathcal{Q}$ это минимальный элемент, и семья $\mathcal{F}$ упорядочена лексикографически. В силу консистентности $\mathcal{S}$ весь интервал между $\tau(Q)=T-n$ и $\tau(S)$ принадлежит $\mathcal{S}$, и, в частности, множество $\tau(R)$. Так что $R$ принадлежит $\mathcal{S}$.

Аналогично рассматривается случай, когда $Q$ лежит после перегородки. В этом случае $Q$ - максимальный элемент (относительно ограничения $\preceq$ на семью $\mathcal{F}$ ), и $\tau(Q)$ не принадлежит $\mathcal{S}$. Значит естественный порядок $\preceq$ на $\mathcal{F}$ антилексикографический, в нем $R$ идет после $S$, и снова из консистентности $R$ принадлежит $\mathcal{S}$.

Таким образом, мы имеем два эквивалентных (лучше сказать - криптоморфных) описания кубильяжей $Z(n, d)$ : как (минимальных) допустимых порядков на $G r(n, d)$ (Манин-Щехтман) и как консистентных подмножеств в $G r(n, d+1)$ (Циглер). Имея такие описания, можно переписывать на соответствующий язык разные понятия и конструкции с кубильяжами. Например, повышающий флип соответствует увеличению консистентного множества на один элемент. Так что отношение $\leq$ для кубильяжей (см. раздел 8) согласовано с отношением включения для консистентных множеств: если $\mathcal{Q} \leq \mathcal{Q}^{\prime}$, то $\operatorname{Inv}(\mathcal{Q}) \subset \operatorname{Inv}\left(\mathcal{Q}^{\prime}\right)$. Обратное свойство верно, если $d=1$ (классической свойство слабого порядка Брюа) или $d=2$ ([22, 23]), но нарушается для больших $d$ (Циглер в [10] привел контрпример при $d=3$ ). В следующем разделе мы приведем еще одно описание (инициированное Леклерком и Зелевинским [6] и развитое Галашиным и Постниковым [12]), в терминах систем $S p(\mathcal{Q})$ спектров вершин кубильяжа. 


\section{6 Отношение разделенности}

Напомним, что с каждой вершиной $v$ кубильяжа $\mathcal{Q}$ зонотопа $Z(n, d)$ мы связывали подмножество $s p(v)$ в $[n]$ - спектр вершины. Когда $v$ пробегает все вершины $\mathcal{Q}$, мы получаем систему $S p(\mathcal{Q})$, подмножество в $2^{[n]}$. В разделе 4 было показано, что система $S p(\mathcal{Q})$ однозначно определяет кубильяж $\mathcal{Q}$. Поэтому естественно встает вопрос - какими свойствами обладают такие системы?

Первое свойство - это ее размер. В разделе 2 было показано, что размер системы $S p(\mathcal{Q})$ равен $\left(\begin{array}{c}n \\ \leq d\end{array}\right)$. Этого, конечно, мало, чтобы характеризовать такие системы. Второе важное структурное свойство состоит в том, что любые два множества из $S p(\mathcal{Q})$ являются $(d-1)$-разделенными.

Определение. Два подмножества $X$ и $Y$ в $[n]$ называются $r$-разделенными $(r$ - натуральное число), если не существует возрастающей цепочки (размера $r+2$ ) $i_{0}<i_{1}<\ldots<i_{r+1}$, такой что элементы с четными номерами лежат в одном из множеств $X-Y$ или $Y-X$, а элементы с нечетными - в другом. Это симметричное и рефлексивное отношение обозначим как $S_{r}$. Система подмножеств называется $r$-разделенной, если любые два ее члена $r$-разделены.

Иными словами, $X$ и $Y r$-разделены, если множество $[n]$ можно разбить на $r+1$ интервал $I_{0}, \ldots, I_{r}$ (некоторые могут быть пустыми), так что $X-Y$ лежит в одной части этих интервалов, а $Y-X-$ в другой части.

Такие интервалы удобно задавать 'разделителями' (сепараторами), то есть $r$ точками $c_{1} \leq c_{2} \leq \ldots \leq c_{r}$ на вещественной прямой, так что интервал $I_{i}$ состоит из целых точке $x$, удовлетворяющих неравенствам $c_{i}<x<c_{i+1}$ (считая $c_{0}=0$, $\left.c_{r}=n+1\right)$.

Отношения $S_{r}$ слабеют с ростом $r, S_{0} \subset S_{1} \subset \ldots \subset S_{n}$. Рассмотрим несколько первых членов этой серии отношений.

Пример 0. Отношение $X S_{0} Y$ означает, что множества $X$ и $Y$ сравнимы по включению: $X \subset Y$ или $Y \subset X$. Можно также сказать, что отношение $S_{-1}$ это отношение равенства $=$.

Пример 1. $S_{1}$ - это отношение сильной разделенности, введенное Леклерком и Зелевинским в [6]. Оно означает, что после удаления общей части либо $X$ располагается левее $Y(X<Y)$, либо $Y$ располагается левее $X$.

Пример 2. $S_{2}$ - это отношение хорд-разделенности, исследованное в [11].

Отношения разделенности $S_{r}$ имеют непосредственное отношение к кубильяжам. Это показывает

Предложение 15 Пусть $\mathcal{Q}$ - кубильяљ зонотопа $Z(n, d)$. Тогда для любых двух вершин $v$ и $w$ кубильяжа $\mathcal{Q}$ множества $\operatorname{sp}(v)$ u $s p(w)(d-1)$-разделеньь.

Иными словами, система $S p(\mathcal{Q})(d-1)$-разделенная. Например, для любого ромбического тайлинга $\mathcal{T}$ система $S p(\mathcal{T}) 1$-разделена - факт, замеченный в [6]. Или другой, крайний случай. Рассмотрим, как в разделе 7, куб $Z(d, d)$. У него есть две 'выступаюшие' точки - хвост $t$ и голова $h$. Спектр головы $-\{d, d-2, \ldots\}$, спектр 
хвоста $-\{d-1, d-3, \ldots\}$, В совокупности эти элементы, чередуясь, покрывают все $[n]$. Поэтому множества $s p(h)$ и $s p(t)$ являются $(d-1)$-разделенными (но не $(d-2)$-разделенными). Если же мы 'сплющим' (спроектируем) этот куб в капсид $Z(d, d-1)$, точка $t$ превратится во внутреннюю (центральную) точку стандартного кубильяжа зонотопа $Z(d, d-1)$ и, как легко проверить, будет $(d-2)$-разделена со всеми другими вершинами этого кубильяжа. Но она не $(d-2)$-разделена с проекцией точки $h$, центральной точкой другого, антистандартного кубильяжа.

Доказательство предложения 15. Предположим, что $X=s p(v)$ и $Y=s p(w)$ не $(d-1)$-разделены. Тогда существуют элементы-цвета $i_{0}<i_{1}<\ldots<i_{d}$, такие, что $i_{0}, i_{2}, \ldots$ принадлежат, скажем, $X-Y$, а $i_{1}, i_{3}, \ldots$ принадлежат $Y-X$. Делая редукцию по всем цветам, отличным от $i_{0}, \ldots, i_{d}$, мы получаем кубильяж капсида $Z(d+1, d)$. И в нем две точки $v^{\prime}$ и $w^{\prime}$ (образы $v$ и $w$ при редукции) со спектрами $i_{0}, i_{2}, \ldots$ и $i_{1}, i_{3}, \ldots$ Но, как мы видели выше, эти точки лежат в разных кубильяжах, одна - в стандартном ( $v^{\prime}$ при нечетном $d$ и $w^{\prime}$ при четном), а другая - в антистандартном.

В следующем Предложении мы опишем спектр стандартного (и антистандартного) кубильяжей зонотопа $Z(n, d)$.

Предложение 16 Пусть $v$ - вершина стандартного кубильяжа зонотопа $Z(n, d)$. Тогда существует разложение $[n]=I_{0} \sqcup I_{1} \sqcup \ldots \sqcup I_{d}$ на $d+1$ последовательныи интервал, так что $s p(v)=I_{d-1} \sqcup I_{d-3} \sqcup \ldots$ Обратно, любое множество такого вида есть спектр некоторой вершины стандартного тайлинга.

Для антистандартного кубильяжа спектры вершин имеют вид $I_{d} \sqcup I_{d-2} \sqcup \ldots$.

Доказательство. Стандартный кубильяж зонотопа $Z(n, d)$ получается из стандартного же кубильяжа $Z(n-1, d)$ путем экспансии (добавления) цвета $n$ вдоль задней (невидимой) мембраны (см. Пример из раздела 6). Поэтому спектр стандартного кубильяжа состоит из двух частей - первая состоит из множеств вида $I_{d-1} \cup I_{d-3} \cup \ldots$, где интервалы $I_{d}, I_{d-1}, \ldots, I_{0}$ берутся из $[n-1]$, а вторая получается из спектров вершин 'задней' мембраны (невидимой части границы зонотопа $Z(n-1, d))$ добавлением цвета $n$. Но 'задняя' мембрана - это фактически антистандартный кубильяж зонотопа $Z(n-1, d-1)$, и спектры ее вершин имеют вид $J_{d-1} \cup J_{d-3} \cup \ldots$, где интервалы $J_{d-1}, \ldots, J_{0}$ снова берутся из $[n-1]$. В первом случае нужно 'подправить' интервал $I_{d}$, заменив его на интервал $I_{d} \cup\{n\}$ (заметим, что $I_{d}$ либо содержит цвет $n-1$, либо пуст). Во втором случае мы добавляем новый интервал $J_{d}=\{n\}$.

$\mathrm{K}$ примеру, для $d=1$ спектр стандартного кубильяжа $Z(n, 1)$ состоят из $(n+1)$ го интервала $[k], k=0,1, \ldots, n$. Соотвественно, спектры антистандартного тайлинга состоят из дополнительных интервалов: $[n],[n-1 . . n], \ldots,[n-k . . n], \ldots, \emptyset$.

Для $d=2$ спектр стандартного ромбического тайлига состоит из произвольных интервалов в $[n]$, тогда как спектр антистандарного тайлинга - из коинтервалов (дополнений к интервалам).

Для $d=3$ спектр стандартного кубильяжа состоят из т.н. 'полуторных' интервалов, то есть множеств вида $[1, i] \cup[j, k]$, где $0 \leq i \leq j \leq k-1 \leq n$. 
Как следствие Предложения 16 мы получаем описание спектров периферийных вершин, то есть вершин зонотопа. Это в точности вершины, которые принадлежат одновременно стандартному и антистандартному кубильяжам. Тут удобно ввести понятие интервального ранга подмножества $X \subset[n]$ как минимального числа $r$, такого что $X$ представляется как объединение интервалов $I_{1} \sqcup \ldots \sqcup I_{r}$.

Следствие 1. Множество X является спектром периферийной вериины зонотопа $Z(n, d)$ тогда и только тогда, когда сумма интервальных рангов $X$ u дополнительного множества $[n]-X$ не больше $d$.

В частности, если размер некоторого множества $X$ не больше, чем $\frac{d-1}{2}$ (то есть $2|X| \leq d-1)$, то соответствующая точка $v(X)$ является периферийной, то есть вершиной зонотопа $Z(n . d)$. Иными словами, внутренние (не лежащие на границе зонотопа) целые точки появляются только на высоте $h \geq d / 2$ и выше. Этот факт тесно связан с явлением, обычно упоминаемым при обсуждении циклических политопов (см., например, [5, Пример 0.6]). А именно, что любое множество вершин циклического политопа размера не больше $\frac{d-1}{2}$ принадлежит некоторой грани этого политопа.

Следствие 2. Спектр периферийной вершины $(d-1)$-разделен с любым подмножеством в $[n]$.

Это утверждение коррелирует с Предложением 3, которое утверждает, в частности, что любая 'целая' точка вписывается в некотрый кубильяж.

\section{7 Разделенность и кубильяжи}

Мы уже получили, что система $S p(\mathcal{Q})$ для кубильяжа $\mathcal{Q}$ зонотопа $Z(n, d)$ является $(d-1)$-разделенной и имеет размер $\left(\begin{array}{c}n \\ \leq d\end{array}\right)$. Теперь пойдем в обратном направлении и покажем, что система с такими свойствами получается как спектр некоторого кубильяжа. Для начала покажем, что число $\left(\begin{array}{c}n \\ \leq d\end{array}\right)-$ это верхняя граница для размера $(d-1)$-разделенной системы в $[n]$.

Предложение 17 Пусть $\mathcal{S}-(d-1)$-разделеннал система подмножеств в $[n]$. Тогда ее размер не превышает $\left(\begin{array}{c}n \\ \leq d\end{array}\right)$.

Доказательство. Разобьем $\mathcal{S}$ на две части: $\mathcal{S}_{0}$, члены которой не содержат цвет $n$, и $\mathcal{S}_{1}$, члены которой содержат $n$. Обозначим через $\mathcal{S}_{2}$ систему множеств вида $X-n$, где $X$ пробегает $\mathcal{S}_{1}$. Очевидно, что размер $\mathcal{S}_{2}$ равен размеру $\mathcal{S}_{1}$. Легко понять, что $\mathcal{S}_{0} \cup \mathcal{S}_{2}-(d-1)$-разделенная система в $[n-1]$, поэтому (по индукции) ее размер не превосходит $\left(\begin{array}{c}n-1 \\ \leq d\end{array}\right)$.

Рассмотрим теперь пересечение $\mathcal{T}=\mathcal{S}_{0} \cap \mathcal{S}_{2}$. Оно состоит из множеств $X \subset$ $[n-1]$, которые принадлежат $\mathcal{S}$, причем $X n$ тоже принадлежит $\mathcal{S}$. Мы утверждаем, что система $\mathcal{T}(d-2)$-разделенная. В самом деле, предположим, что в $\mathcal{T}$ есть множества $X$ и $Y$, которые не $(d-2)$-разделенные. Значит есть последовательность $i_{0}<i_{1}<\ldots<i_{d-1}$, такая, что члены с четными индексами принадлежат (скажем) 
$X-Y$, а с нечетными $-Y-X$. Но тогда последовательность $i_{0}<i_{1}<\ldots \lesssim i_{d-1}<$ $i_{d}=n$ обладает тем же свойством либо для $\widetilde{X}=X n$ и $Y$, либо для $X$ и $\widetilde{Y}=Y n$. Это противоречит предположению о $(d-1)$-разделенности $\mathcal{S}$.

Снова по индуктивному предположению размер $\mathcal{T}$ не превосходит $\left(\begin{array}{c}n-1 \\ \leq d-1\end{array}\right)$. Отсюда размер $\mathcal{S}$, равный сумме размеров $\mathcal{S}_{0}$ и $\mathcal{S}_{2}$, то есть сумме размеров $\mathcal{S}_{0} \cup \mathcal{S}_{2}$ и $\mathcal{S}_{0} \cap \mathcal{S}_{2}$, не превосходит $\left(\begin{array}{c}n-1 \\ \leq d\end{array}\right)+\left(\begin{array}{c}n-1 \\ \leq d-1\end{array}\right)=\left(\begin{array}{c}n \\ \leq d\end{array}\right)$.

Ввиду Предложения $17(d-1)$-разделенные системы в $[n]$ размера $\left(\begin{array}{c}n \\ \leq d\end{array}\right)$ называают максимальными по размеру, противопоставляя это максималъности по включению. Следующий результат установлен Галашиным и Постниковым [12] в несколько большей общности (они не ограничивались кубильяжами циклических зонотопов).

Теорема 5 Пусть $\mathcal{S}-(d-1)$-разделенная система в $[n]$ размера $\left(\begin{array}{c}n \\ \leq d\end{array}\right)$. Тогда она имеет вид $S p(\mathcal{Q})$ для некоторого (единственного) кубильяжа зонотопа $Z(n, d)$.

Доказательство. Оно близко к доказательству Предложения 17. Снова разделим $\mathcal{S}$ на $\mathcal{S}_{0}$ и $\mathcal{S}_{1}$ и обозначим $\mathcal{S}_{2}$ как выше. Тогда из доказательства Предложения 17 следует, что размер $\mathcal{S}_{0} \cup \mathcal{S}_{2}$ равен $\left(\begin{array}{c}n-1 \\ \leq d\end{array}\right)$, а размер пересечения $\mathcal{S}_{0} \cap \mathcal{S}_{2}$ равен $\left(\begin{array}{c}n-1 \\ \leq d-1\end{array}\right)$. По индукции мы можем считать, что система $\mathcal{S}_{0} \cup \mathcal{S}_{2}$ реализуется некоторым кубильяжем $\mathcal{Q}^{\prime}$ в $Z(n-1, d)$, а пересечение $\mathcal{S}_{0} \cap \mathcal{S}_{2}-$ кубильяжем $\mathcal{Q}^{\prime \prime}$ зонотопа $Z(n-$ $1, d-1)$. Очевидно, что $\mathcal{Q}^{\prime \prime}$ реализуется мембраной $\mathcal{M}$ в кубильяже $\mathcal{Q}^{\prime}$. Остается взять в качестве $\mathcal{Q}$ экспансию по цвету $n$ кубильяжа $\mathcal{Q}^{\prime}$ вдоль этой мембраны. На уровне спектров это сведется к замене всех множеств $X$ из $\mathcal{S}_{2}$ на множества $X n$. Так что $S p(\mathcal{Q})=\mathcal{S}_{0} \cup \mathcal{S}_{1}=\mathcal{S}$.

Таким образом, мы получаем еще одно криптоморфное воплощение кубильяжей. И снова можно в этих новых терминах пересказывать разные конструкции.

Приведем еще один интересный пример разделенных систем.

Предложение 18 Пусть $\mathcal{M}_{1}$ и $\mathcal{M}_{2}$ - две мембраны в зонотопе $Z(n, d)$, причем $\operatorname{Inv}\left(\mathcal{M}_{1}\right) \subset \operatorname{Inv}\left(\mathcal{M}_{2}\right)$. Тогда система $\operatorname{Sp}\left(\mathcal{M}_{1}\right) \cup \operatorname{Sp}\left(\mathcal{M}_{2}\right)(d-1)$-разделена.

Доказательство. Пусть это не так, и некоторые множества $X \in S p\left(\mathcal{M}_{1}\right)$ и $Y \in$ $S p\left(\mathcal{M}_{2}\right)$ не $(d-1)$-разделены. Тогда сушествует последовательность элементов $i_{1}<i_{2}<\ldots<i_{d+1}$ из $[n]$, которые поочередно лежат то в $X-Y$, то в $Y-$ $X$. Рассмотрим случай, когда в $X-Y$ лежат элементы $i_{d+1}, i_{d-1}, \ldots$ (а элементы $i_{d}, i_{d-2}, \ldots$ лежат в $\left.Y-X\right)$, и образуем $d$-элементное множество $K=\left\{i_{1}, \ldots, i_{d}\right\}$. По Лемме 3, примененной к $X$, мы получаем, что $K$ является инверсным для мембраны $\mathcal{M}_{1}$, а значит и для мембраны $\mathcal{M}_{2}$. Но по той же Лемме 3 мы получаем, что множество $X$ неинверсное для мембраны $\mathcal{M}_{2}$.

Аналогично разбирается случай, когда элементы $i_{d+1}, i_{d-1}, \ldots$ лежат в $Y-X$. Однако теперь мы образуем $d$-элементное множество $K^{\prime}=\left\{i_{2}, \ldots, i_{d+1}\right\}$. По Лемме3 $K^{\prime}$ неинверсное для мембраны $\mathcal{M}_{2}$, а значит и для $\mathcal{M}_{1}$. Но по той же лемме $K^{\prime}$ инверсное для $\mathcal{M}_{1}$. 


\section{8 Чистота и расширяемость}

Предложение 17 поднимает следующий вопрос - когда данная $(d-1)$-разделенная система $\mathcal{S}$ подмножеств в $[n]$ расширяется до системы максимального размера, то есть до $(d-1)$-разделенной системы размера $\left(\begin{array}{c}n \\ \leq d\end{array}\right)$ ? Или, в терминах раздела 4 , когда соответствующее множество целых точек зонотопа $Z(n, d)$ вписывается в некоторый кубильяж этого зонотопа. Если это так, система $\mathcal{S}$ называется расииряемой (или, точнее, $(n, d)$-расширяемой). Если любая $(d-1)$-разделенная система расширяема, то говорят, что для пары $(n, d)$ выполнено свойство чистоты.

Например, мы видели в разделе 4, что любая одноэлементная система (то есть любое подмножество в $[n]$ ) расширяема. Согласно Теореме 2 из раздела 13 любая мембрана вписывается в кубильяж. Покажем, что любая двухэлементная система расширяема.

Предложение 19 Пусть $X$ и $Y$ - два $(d-1)$-разделенных подмножества в $[n]$. Тогда двухэлементная система $\{X, Y\}$ расширяема.

Доказательство. Будем доказывать индукцией по $n$. Предположим, что цвет $i$ не входит в $X$ и $Y$. Тогда их можно рассматривать как подмножества в $[n]-i$. По индукции множества $X$ и $Y$ вписываются в кубильяж $\mathcal{Q}$ зонотопа $Z([n]-i, d)$. Остается сделать экспансию по цвету $i$ (см. раздел 4) относительно невидимой (в направлении $v_{i}$ ) части границы этого зонотопа.

Аналогично рассуждаем в том случае, когда цвет $i$ входит как в $X$, так и в $Y$. Только теперь надо делать экспансию по цвету $i$ относительно видимой по этому цвету части границы зонотопа.

Таким образом, все сводится к случаю, когда множества $X$ и $Y$ дополнительны друг к другу в $[n]$. Пользуясь интервальным представлением, мы видим, что $X$ и $Y$ образованы из чередующихся интервалов в некотором разложении $I_{0} \sqcup I_{1} \sqcup$ $\ldots \sqcup I_{r}$ цепи $[n]$ на последовательно идущие непустые интервалы. В силу $(d-1)$ разделенности $X$ и $Y$ мы имеем $r<d$. Но это означает, что $X$ и $Y$ (точнее, реализующие их точки $v(X)$ и $v(Y))$ принадлежат 'периферии' зонотопа $Z(n, d)$ и потому вписываются в любой кубильяж.

Как мы увидим в следующем разделе, это утверждение уже неверно для трех множеств. Это означает, что свойство чистоты выполнено не для любых $(n, d)$. А для каких же?

Тривиально чистота имеет место для $d=1$. Чистота для $d=2$ была установлена Леклерком и Зелевинским в семинальной работе [6] (см. также наш обзор [7]). Чистота для $d=3$ установлена в работе Галашина [11]. Кроме того, по тривиальным причинам чистота имеет место для $n=d+1$ (капсиды). Этими случаями и исчерпывается чистота, как показано в [12]. В следующем разделе мы более подробно рассмотрим случай $n=d+2$ при $d \geq 4$ и приведем простые и явные примеры нерасширяемых систем. Для распространения таких результатов на случай $n>d+2$ полезны будут следующие два факта.

Предложение 20 Пустъ $\mathcal{S} \subset 2^{[n]}$. Система $\mathcal{S}(n, d)$-расширяема тогда и только тогда, когда она (рассмотренная как система подмножеств в $[n+1])(n+1, d)$ расширяема. 
Доказательство. Пусть $\mathcal{S}(n, d)$-расширяема. Тогда она $(d-1)$-разделенная и реализуется как подмножество вершин некоторого кубильяжа $\mathcal{Q}$ зонотопа $Z=$ $Z(n, d)$. Пусть кубильяж $\mathcal{Q}^{\prime}$ зонотопа $Z(n+1, d)$ получен из $\mathcal{Q}$ экспансией по цвету $n+1$ относительно невидимой (задней) мембраны $Z$. Так как $\mathcal{Q} \subset \mathcal{Q}^{\prime}$, то $\mathcal{S}$ вписывается в $\mathcal{Q}^{\prime}$.

Обратно, пусть $\mathcal{S}$ вписывается в некоторый кубильяж $\mathcal{Q}^{\prime}$ зонотопа $Z(n+1, d)$. И пусть $\mathcal{P}=\mathcal{P}_{n+1}-$ перегородка цвета $n+1$ в $\mathcal{Q}^{\prime}$. Так как множества из $\mathcal{S}$ не содержат цвет $n+1$, эта система вершин лежит (нестрого) до перегородки $\mathcal{P}$. Поэтому при редукции $\mathcal{P}$ мы получим кубильяж $\mathcal{Q}$ зонотопа $Z$, в который $\mathcal{S}$ вписывается.

Предложение 21 Пусть $\mathcal{S}-(d-1)$-разделенная система подмножеств в $[n]$, u $n^{\prime}=n+1$. Образуем систему $\mathcal{S}^{\prime} \subset 2^{[n+1]}, \mathcal{S}^{\prime}=\left\{X n^{\prime}, X \in \mathcal{S}\right\}$. Cистема $\mathcal{S}(n, d)$ расширяема тогда и толъко тогда, когда система $\mathcal{S} \cup \mathcal{S}^{\prime}(n+1, d+1)$-расширяема.

Доказательство. Пусть $\mathcal{S}(n, d)$-расширяемая, то есть вписывается в некоторый кубильяж $\mathcal{Q}$ зонотопа $Z(n, d)$. Реализуем $\mathcal{Q}$ как мембрану $\mathcal{M}$ в некотором кубильяже $\mathcal{Q}^{\prime}$ зонотопа $Z(n, d+1)$, так что $\mathcal{S}$ образует часть вершин $\mathcal{M}$. Пусть, наконец, $\mathcal{Q}^{\prime \prime}$ - экспансия $\mathcal{Q}^{\prime}$ по цвету $n^{\prime}$ относительно этой мембраны $\mathcal{M}$; это некоторый кубильяж зонотопа $Z(n+1, d+1)$. При этом мембрана $\mathcal{M}$ расширяется до перегородки $\mathcal{P}$ цвета $n^{\prime}$ в $\mathcal{Q}^{\prime \prime}$. Система $\mathcal{S}$ лежит на одной (видимой) стороне этой перегородки, тогда как $\mathcal{S}^{\prime}$ лежит на другой (невидимой) стороне перегородки $\mathcal{P}$. Откуда видно, что $\mathcal{S} \cup \mathcal{S}^{\prime}$ вписывается в $\mathcal{Q}^{\prime \prime}$.

Обратно, пусть система $\mathcal{S} \cup \mathcal{S}^{\prime}$ (как множество целых точек в зонотопе $Z^{\prime \prime}=$ $Z(n+1, d+1))$ вписывается в некоторый кубильяж $\mathcal{Q}^{\prime \prime}$ зонотопа $Z^{\prime \prime}$. Рассмотрим перегородку $\mathcal{P}$ цвета $n^{\prime}$ в $\mathcal{Q}^{\prime \prime}$. Ясно, что $\mathcal{S}$ располагается на одной стороне этой перегородки, тогда как $\mathcal{S}^{\prime}$ - на другой (и получается из $\mathcal{S}$ сдвигом на вектор $v_{n^{\prime}}$ ). Редукция этой перегородки приводит к кубильяжу $\mathcal{Q}^{\prime}$ зонотопа $Z^{\prime}=Z(n, d+1)$; при этом перегородка $\mathcal{P}$ сжимается до мембраны $\mathcal{M}$, а системы $\mathcal{S}$ и $\mathcal{S}^{\prime}$ сливаются в одну систему $\mathcal{S}$ точек на этой мембране. Остается спроектировать эту мембрану и получить кубильяж $\mathcal{Q}$ зонотопа $Z(n, d)$, содержаций систему $\mathcal{S}$ как подмножество вершин.

Следствие. Пусть $\mathcal{S}$ - нерасширяемая система в зонотопе $Z(d+r, d), r \leq r^{\prime}$ $u d \leq d^{\prime}$. Тогда существует нерасширяемая система в зонотопе $Z\left(d^{\prime}+r^{\prime}, d^{\prime}\right)$.

Мы говорили выше, что любая мембрана вписывается в кубильяж. Покажем, что не любая пара мембран (даже $(d-1)$-разделенных) вписываются в кубильяж. Воспользуемся примером, построенным Циглером [10]. А именно, он построил две мембраны $\mathcal{M}_{1}$ и $\mathcal{M}_{2}$ в зонотопе $Z(8,4)$ (он задавал их в форме консистентных систем), которые обладали двумя свойствами: 1) $\operatorname{Inv}\left(\mathcal{M}_{1}\right) \subset \operatorname{Inv}\left(\mathcal{M}_{2}\right)$, и 2) эти две мембраны не вписываются в один кубильяж зонотопа $Z(8,4)($ справедливости ради надо сказать, что Циглер формулировал свойство 2) немного в других терминах). Так вот, в силу Предложения 19 объединение спектров $\mathcal{M}_{1}$ и $\mathcal{M}_{2}$ является 3 -разделенной системой в $G r([8], 4)$, но не продолжающейся до максимальной по размеру. Это также дает пример отсутствия чистоты. 


\section{9 Случай $Z(6,4)$}

Здесь мы более подробно исследуем вопрос о расширяемых/нерасширяемых системах точек в $Z(d+2, d)$. Мы уже касались в разделе 11 вопроса о кубильяжах таких зонотопов и выяснили, что их бывает $2 n$ штук, где $n=d+2$. Мы подробно рассмотрим $Z(6,4)$ (в случае $Z(5,3)$ все 2-разделенные подсистемы расширяемы). На этом простом примере видны почти все эффекты общего случая $Z(d+2, d)$.

При малом $n-d$ почти все целые точки лежат на границе зонотопа $Z(n, d)$. Например, при $n=d+1$ только две точки не на границе. В случае $n=d+2$ непериферийных точек $2 n$. В самом деле, по формуле (1.2) число периферийных точек равно $2\left(\left(\begin{array}{c}d+1 \\ d-1\end{array}\right)+\ldots+\left(\begin{array}{c}d+1 \\ 0\end{array}\right)\right)=2\left(2^{d+1}-1-(d+1)\right)=2^{n}-2 n$. И так как все периферийные точки $(d-1)$-разделены со всеми точками, то нам надо разобраться с разделенностью этих $2 n$ непериферийных точек-множеств. Здесь мы и начнем считать, что $n=6$, а $d=4$.

В этом случае имеется $52=64-12$ периферийных точек-множеств. Выпишем явно 12 непериферийных точек-множеств. Как было показано в Следствии 1 Предложения 16 , периферийные точки задаются разбиением отрезка $[n]$ на $d$ последовательно идущих интервала; после этого надо взять объединение либо 'четных' интервалов, либо 'нечетных'. В случае, когда $d=4$, а $n=6$, непериферийные точки-множества - это те, которые не укладываются (альтернированно) в четыре интервала, а должны задействовать 5 или 6 (непустых) интервалов. Понятно, что такие интервалы либо одноэлементные (и тогда их шесть), либо почти все одноэлементные, и только один интервал двухэлементный (и тогда интервалов пять). Выпишем эти 12 множеств как строки сдедующей таблицы.

$\begin{array}{llllll}1 & & 3 & & 5 & \\ 1 & & 3 & & 5 & 6 \\ 1 & & 3 & & & 6 \\ 1 & & 3 & 4 & & 6 \\ 1 & & & 4 & & 6 \\ 1 & 2 & & 4 & & 6 \\ & 2 & & 4 & & 6 \\ & 2 & & 4 & & \\ & 2 & & 4 & 5 & \\ & 2 & & & 5 \\ & 2 & 3 & & 5 & \\ & & 3 & & 5 & \end{array}$

Эти 12 множеств удобно расположить по кругу, как на цифербате часов, помещая 135 на месте шести часов, а дополнительное множество 246 на месте двенадцати часов. См. рис. 16.

Какие же множества среди этих 12-ти 3-разделенные? Легко понять, что неразделенными является множество и его дополнение, а также два соседа этого дополнения. Так что неразделенными являются множество и три 'противоположных' к нему. Это можно увидеть также из того, что подряд идущие 5 множеств на циферблате вписываются в один кубильяж (и дают все 12 кубильяжей зонотопа 


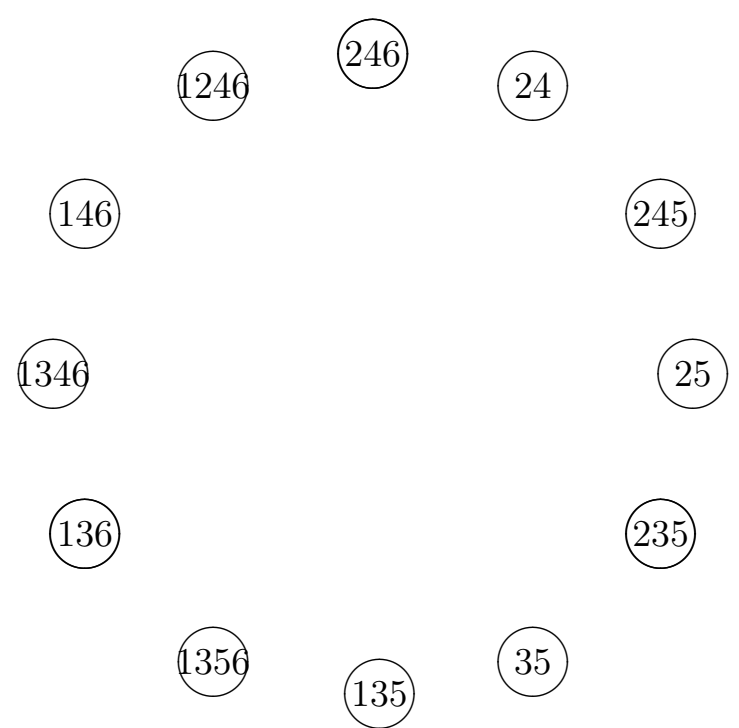

Рис. 16: циферблат

$Z(6,4)$ ). Например, 'цифры' от часа до пяти (а именно, множества 24, 245, 25, 235 и 35$)$ вписываются в стандартный кубильяж. Из этого описания 3-неразделенности видно, что три множества (размещенные как XII часов, IV часа и VIII часов, то есть множества 246, 235 и 136) образуют (вместе с периферией) максимальную по включению 3 -разделенную систему из $52+3=55$ множеств, тогда как максимум по размеру равен $52+5=57$. Это показывает, что в $Z(6,4)$ имеется система размера 55 , которая не $(6,4)$-расширяемая (и максимальна по включению).

Аналогичные рассуждения показывают, что в $Z(d+2, d)$ (при $d \geq 4$ ) имеется $(d+2, d)$-нерасширяемая система 4 Любопытно отметить, что в случае $d=8$ на цифеблате из 20 'часов' можно указать 5 'цифр' $(0,4,8,12$ и 16$)$, которые дают максимальную по включению 7-разделенную подсистему в этой 20-элементной непериферийной системе. Вместе с периферией это дает 1009-элементную максимальную по включению 7-разделенную систему подмножеств множества [10]. В то время как максимум по размеру равен $1004+9=1013$. Разница равна $1013-1009=4=d / 2$, что дает основания предположить, что размер максимальной по включению $(d-1)$ разделенной системы отличается от максимума по размеру не более чем на d/2.

Вместе со Следствием из предыдущего раздела мы получаем теорему ГалашинаПостникова [12], что если $d \geq 4 u n \geq d+2$, то существует $(n, d)$-нерасширяемая система множеств в $[n]$. В частности, для таких $(n, d)$ нет чистоты.

\section{0 Слабая разделенность}

В разделе 16 и последующих вводилось и изучалось отношение $k$-разделенности в множестве $2^{[n]}$. Обобщая понятие слабой разделенности Леклерка и Зелевинского

\footnotetext{
${ }^{4}$ Отсутствие чистоты при $d=7$ фактически утверждается в [24, Proposition 8.1].
} 
[6], можно предложить понятие слабой $k$-разделенности в случае нечетного $k$. (Для четного $k$ мы не видим хорошего определения.) Удобнее, однако, задавать это отношение как усиление отношения $(k+1)$-разделенности.

Итак, пусть $k$ - нечетное натуральное число. Напомним, что подмножества $A$ и $B$ в $[n]$ назывались $(k+1)$-разделенными: если существует такое разбиение $[n]$ на $k+2$ последовательных интервала $[n]=I_{0} \sqcup I_{1} \sqcup \ldots \sqcup I_{k+1}$, что множество $A-B$ располагается в объединении 'четных' интервалов $I_{0} \sqcup I_{2} \sqcup \ldots I_{k+1}$, а $B-A-$ в объединении 'нечетных' интервалов $I_{1} \sqcup \ldots I_{k}$ (или наоборот).

Определение. Множества $A$ и $B$ как выше называются сильно $(k+1)$-разделенными (или слабо $k$-разделенными), если размер $A$ не больше, чем размер $B$. Образно говоря, 'окружающее' множество по размеру не превосходит 'окружаемое'.

Например, множество 15 слабо 1-разделено с множествами 234 и 24, но не с множеством 3. Отношение слабой 1-разделенности - это в точности отношение слабой разделенности Леклерка-Зелевинского. Если множества $A$ и $B k$-разделены, то они слабо $k$-разделены, что оправдывает употребление эпитета 'слабо'. Если $A$ и $B$ имеют одинаковый размер, слабая $k$-разделенность превращается в $(k+1)$ разделенность.

В некоторых отношениях слабая $k$-разделенность 'похожа' на $k$-разделенность. Например: в Предложении 17 утверждается, что размер любой $k$-разделенной системы подмножеств $[n]$ не превышает $\left(\begin{array}{c}n \\ \leq k+1\end{array}\right)$. Покажем, что это же верно для слабой $k$-разделенности.

Предложение 22. Пусть $k$ - нечетное число. Размер слабо $k$-разделенной системы подмножеств в $[n]$ не превышает $\left(\begin{array}{c}n \\ \leq k+1\end{array}\right)$.

Доказательство. Оно проводится индукцией по $k$; при $k=-1$ утверждение очевидно, так как сильная 0-разделенность - это просто равенство. Поэтому размер такой системы равен 1 , как и $\left(\begin{array}{l}n \\ 0\end{array}\right)$. При $k \geq 1$ рассуждение начинается как в предложении 17. Пусть $\mathcal{W}$ - слабо $k$-разделенная система в $[n]$; разделим ее на две части: $\mathcal{W}_{0}$, члены которой не содержат цвет $n$, и $\mathcal{W}_{1}$, члены которых содержат цвет $n$. Пусть $\mathcal{W}_{2}$ состоит из множеств вида $X-n$, где $X$ пробегает $\mathcal{W}_{1}$. Ясно, что $\mathcal{W}_{0} \cup \mathcal{W}_{2}$ снова слабо $k$-разделенная система (уже в $\left.[n-1]\right)$, и по индукции ее размер не превосходит $\left(\begin{array}{c}n-1 \\ \leq k+1\end{array}\right)$.

Обозначим через $\mathcal{D}$ систему множеств $X$, которые сами лежат в $\mathcal{W}_{0}$, а их 'двойник' $X n$ лежит в $\mathcal{W}_{1}$. Мы утверждаем, что размер $\mathcal{D}$ не превосходит $\left(\begin{array}{c}n-1 \\ \leq k\end{array}\right)$. Как и в предложении 17, Предложение 22 следует из этой оценки. Следующие три леммы доказывают эту оценку.

Лемма 5. Пусть множества $A$ и $B$ из $\mathcal{D}$ имеют один и тот же размер. Тогда они $(k-1)$-разделены.

Доказательство. $(k-1)$-неразделенность означает, что $(A-B$ и $B-A)$ нельзя альтернированно разместить в разбиении $[n-1]$ на $k$ интервалов. То есть что для их размещения нужно задействовать $k+1$ интервал. Предположим, что такое размещение имеет схему $A B A B \ldots A B$ (слово из $k+1$ буквы). В этом случае сравним $B$ и $A n$. И хотя эти множества помещаются в $k+2$ интервала, размер окружающего множества $A n$ превышает размер окружаемого $B$, что противоречит тому, что 
$A n$ и $B$ слабо $k$-разделены. Аналогично в случае схемы $B A \ldots B A$, где мы будем сравнивать множества $A$ и $B n$.

Обозначим через $\mathcal{D}_{i}$ подсистему тех множеств из $\mathcal{D}$, которые имеют размер $i$. И дополним ее двумя другими системами подмножеств в $[n-1]$. Для их определения рассмотрим две вспомогательные системы $\mathcal{S}$ и $\mathcal{A}$. Чтобы их задать, мы рассмотрим разбиение $[n-1]$ на $k$ интервалов, $[n-1]=I_{0} \sqcup \ldots \sqcup I_{k-1}$. Тогда объединение 'нечетных' интервалов $\left(I_{1} \sqcup \ldots \sqcup I_{k-2}\right)$ этого разбиения дает элемент системы $\mathcal{S}$, а объединение 'четных' интервалов дает элемент системы $\mathcal{A}$. Через $\mathcal{S}_{i}$ мы обозначаем подсистему в $\mathcal{S}$, образованную множествами размера $i$, и аналогично понимаются $\mathcal{A}_{i}$.

Лемма 6. Объединение систем $\mathcal{S}_{n-1} \sqcup \ldots \sqcup \mathcal{S}_{i+1} \sqcup \mathcal{D}_{i} \sqcup \mathcal{A}_{i-1} \sqcup \ldots \sqcup \mathcal{A}_{0}$ образует слабо $(k-2)$-разделенную систему подмножеств множества $[n-1]$.

Доказательство получается их следующих трех простых замечаний. Первое: множество из $\mathcal{S}_{l}$ слабо $(k-2)$-разделено с любым множеством меньшего или равного размера. В самом деле, оно имеет вид $I_{1} \sqcup \ldots \sqcup I_{k-2}$ для некоторого разбиения $[n-1]=I_{0} \sqcup \ldots \sqcup I_{k-1}$ на $k$ интервалов. Условие на размер дает сильную $(k-1)$ разделенность, то есть слабую $(k-2)$-разделенность. Второе: по тем же причинам множество из $\mathcal{A}_{l}$ слабо $(k-2)$-разделено с любым множеством большего или равного размера. Третье - множества из $\mathcal{D}_{i}$ слабо $(k-2)$-разделены по Лемме 5 (они $(k-1)$-разделены и имеют одинаковый размер).

По предположению индукции размер системы $\mathcal{S}_{n-1} \sqcup \ldots \sqcup \mathcal{S}_{i+1} \sqcup \mathcal{D}_{i} \sqcup \mathcal{A}_{i-1} \sqcup \ldots \sqcup \mathcal{A}_{0}$ не превосходит $\left(\begin{array}{c}n-1 \\ \leq k-1\end{array}\right)$. С другой стороны размер системы $\mathcal{S}$ (как спектра стандартного кубильяжа зонотопа $Z(n-1, k-1))$ равен $\left(\begin{array}{c}n-1 \\ <k-1\end{array}\right)$. Откуда мы заключаем, что размер $\mathcal{D}_{i}$ не превосходит разности размеров $\mathcal{S}_{0} \sqcup \mathcal{S}_{1} \sqcup \ldots \sqcup \mathcal{S}_{i}$ и $\mathcal{A}_{0} \sqcup \ldots \sqcup \mathcal{A}_{i-1}$. И осталось разобраться с размером этой разности.

Лемма 7. Эта разность равна числу вериин стандартного (а на самом деле - любого) кубильяжа зонотопа $Z(n-1, k)$ на высоте $i$.

Имея это равенство, мы быстро завершаем доказательство Предложения 22. В самом деле, размер $\mathcal{D}_{i}$ не превосходит числа вершин стандартного кубильяжа на уровне $i$. Поэтому размер всей системы $\mathcal{D}$ не превосходит числа вершин стандартного кубильяжа $Z(n-1, k)$, то есть $\left(\begin{array}{c}n-1 \\ \leq k\end{array}\right)$. Но именно нужно было доказать.

Доказательство леммы 7. Рассмотрим зонотоп $Z=Z(n-1, k)$. Элементы системы $\mathcal{S}$ реализуются как спектры вершины видимой части границы $Z$, элементы $\mathcal{A}$ - как спектры вершин невидимой части границы. Обрежем $Z$ на высоте $i$. Тогда элементы $\mathcal{S}_{0} \sqcup \mathcal{S}_{1} \sqcup \ldots \sqcup \mathcal{S}_{i}$ реалзуются как вершины видимой части этого обрубка ('чаши'), тогда как элементы $\mathcal{A}_{0} \sqcup \ldots \sqcup \mathcal{A}_{i-1}-$ как вершины невидимой части (исключая те, которые находятся на высоте $i$ ).

А теперь возьмем любой кубильяж $Z$, напрмер, стандартный. И рассмотрим гирлянды (см. раздел 7) в этом кубильяже, точнее, те, которые начинаются в точках видимой части границы 'обрубка'. Каждая гирлянда где-то пересекает другую границу 'обрубка', а это либо невидимоя часть обрубка, либо его верхнее 'горизонтальное' основание (потолок). И при этом получается любая вершина кубильяжа 
этой невидимой части границы обрубка. Отсюда мы получаем, что интересующая нас разность в точности равна числу вершин нашего кубильяжа, находящихся на высоте $i$. Лемма 7 доказана, а вместе с ней доказано и Предложение 22.

Наконец, можно поставить вопрос о чистоте отношения слабой разделенности. Для $k=1$ утвердительный ответ был получен в [7]. Однако уже при $k=3$ ответ отрицателен. Для получаеия контрпримера обратимся снова к разделу 19, то есть к системе подмножеств в [6]. В этом случае есть 52 периферийных точек-множеств, которые 3-разделимы с любыми подмножествами в [6]. Мы утверждаем, что

а) Три множества 25, 1356 и 1246 слабо 3-разделены. Они даже 3-разделены, см. раздел 19.

б) Вместе с периферийными точками-множествами эти три множества образуют нерасширяемую дальше (максимальную по включению) слабо 3-разделенную систему размера $52+3=55$. Тогда как $\left(\begin{array}{c}6 \\ \leq 4\end{array}\right)=57$. Утверждение про нерасширяемость проверяется непосредственно. Например, множество 25 4-разделено с множествами 136, 1346 и 146, но разделено не сильно из соображений размера.

Отметим, с другой стороны, что нерасширяемая 3-разделенная система $\{246,235$, $136\}$, рассмотренная как слабо 3-разделенная, допускает расширение за счет добавления двух множеств 146 и 245.

\section{Дополнение 1. Поликатегорный взгляд на кубилья- ЖИ}

Попробуем дать некоторое представление об этой достаточно изощренной конструкции, придуманной Маниным, Шехтманом, Капрановым и Воеводским ([14, 9, 15]).

В основе лежит идея, что кубильяжи можно трактовать как морфизмы, но между чем? Между кубильяжами меньшей размерности. Но те, в свою очередь, можно рассматривать как морфизмы, и так мы приходим не просто к категории, но к поликатегории. Грубо говоря, поликатегория - это система категорий, в которой для любых объектов $a$ и $b$ одной категории множество $\operatorname{Hom}(a, b)$ в свою очередь является множеством объектов другой категории, более высокого уровня.

Чтобы настроиться на этот поликатегорный взгляд, вернемся к ромбическим тайлингам. Пусть есть две мембраны в тайлинге, то есть две змейки, идущие в зоногоне $Z(n, 2)$ из нижней вершины $\emptyset$ в верхнюю вершину $[n]$. Предполагая, что вторая мембрана $\mathcal{M}_{2}$ проходит правее первой $\mathcal{M}_{1}$, мы можем сказать, что $\mathcal{M}_{1} \leq \mathcal{M}_{2}$, если между этими мембранами-змейками можно вписать (частичный) ромбический тайлинг (или, что эквивалентно, обе мембраны вписаны в некоторый ромбический тайлинг). Интерпретируя мембраны-змейки как линейные порядки (или перестановки) на множестве $[n]$, мы получаем (слабый) порядок Брюа.

Однако можно поступить более тонко и сказать, что нас интересует не просто существование тайлингов, 'соединяющих' $\mathcal{M}_{1}$ и $\mathcal{M}_{2}$, но и сами 'соединяющие' их тайлинги. Иначе говоря, морфизмом из $\mathcal{M}_{1}$ в $\mathcal{M}_{2}$ объявляется произвольный (частичный) тайлинг $\mathcal{T}$ между $\mathcal{M}_{1}$ и $\mathcal{M}_{2}$. Так мы получаем вместо посета Брюа 
категорию Брюа, в которой объектами служат перестановки (или линейные порядки на $[n])$, морфизмами - соединяющие их тайлинги, а композиция морфизмов производится просто путем объединения тайлингов. На самом деле, мы получаем большее: ведь два тайлинга $\mathcal{T}_{1}$ и $\mathcal{T}_{2}$ между $\mathcal{M}_{1}$ и $\mathcal{M}_{2}$ тоже можно сравнивать с помощью повышающих флипов. Так что $\operatorname{Hom}\left(\mathcal{M}_{1}, \mathcal{M}_{2}\right)$ не просто (довольно богатое) множество, но тоже посет! И композиция морфизмов-тайлингов уважает эти структуры посетов.

Но если уж мы встали на этот путь, то грех останавливаться. Можно не просто говорить, что тайлинги $\mathcal{T}_{1}$ и $\mathcal{T}_{2}$ соединяются повышающими флипами, но само такое соединение назвать морфизмом (уже следующего, второго уровня). А соединение - это некоторый (частичный) кубильяж уже в зонотопе $Z(n, 3)$. И эту конструкцию можно продолжать и далее, поднимаясь все выше и выше по размерности, пока мы не дойдем до зонотопа-куба $Z(n, n)$, в котором имеется уже только один тривиальный кубильяж.

Так выглядит эта поликатегорная картина в общих чертах.

В следующем примере мы приведем лишь наиболее интересный фрагмент поликатегорного полотна, да и то в предельно простом случае. А именно, возьмем $n=3$, и изобразим лишь первый, второй и третий этажи получающейся 'пагоды'.

Объектами первого этажа служат вершины куба $Z(3,3)$, то есть подмножества базисного множества $[3]=\{1,2,3\}$. Но что считать морфизмами? Казалось бы, естественно полагать, что из множества $X$ идет стрелка в $Y$, если $X \subset Y$. На этом пути мы приходим просто к посету $2^{[3]}$. Кубильяжная точка зрения говорит, что морфизмом надо считать 'путь' от $X$ в $Y$, частичную змейку из $X$ в $Y$. Композиция змеек очевидна: если есть змейка из $X$ в $Y$ и из $Y$ в $Z$, надо просто приставить их друг к другу и получить змейку из $X$ в $Z$.
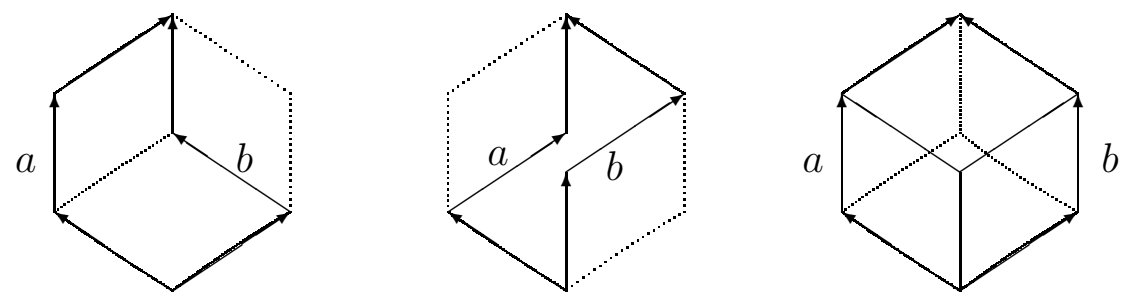

Рис. 17: Змейки в зоногоне $Z(3,2)$

Объектами второго этажа являются частичные змейки. Если есть две змейки $a$ и $b$ с одним и тем же началом и одним и тем же концом, возникает вопрос- можно ли замостить промежуток между ними ромбами? И морфизмами второго этажа (между $a$ и $b$ ) являются именно такие ромбические замощения области между $a$ и $b$ (здесь надо считать, что змейка $b$ проходит правее змейки $a$ ). В нашем случае с $n=3$ такое замощение бывает либо одно (как слева на рис. 17), или ни одного (как на среднем рисунке), где змейки идут из $\emptyset$ в [3]).

Однако (в нашем простом случае) есть единственное исключение, когда между змейкой 123 и 321 (здесь мы понимаем сокращение 123 как линейный порядок на 
множестве $\{1,2,3\})$ возможны два замощения или тайлинга: стандартный и антистандартный. Так что если нарисовать часть второго этажа, содержащую только 'полные' змейки (из Ø в [3], то есть шесть линейных порядков на множестве [3]), мы получим следущую картину (рис. 18).

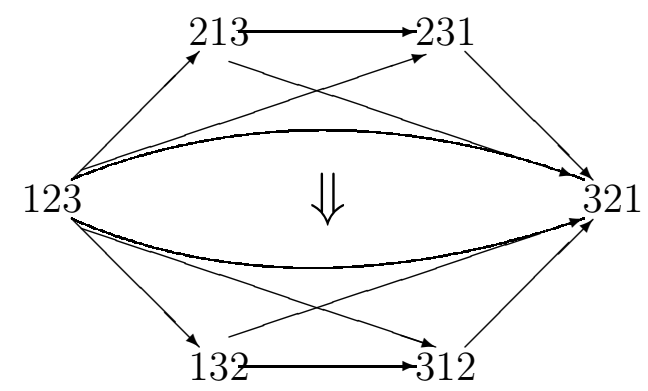

Рис. 18: Частичная картина поликатегории в случае $n=3$.

Мы хотели бы обратить внимание, что из змейки 123 в змейку 321 идут два морфизма-тайлинга: стандартный и антистандартный. И эти два морфизма, как объекты третьего этажа, тоже связаны морфизмом (третьего уровня), который изображен двойной стрелкой $\Rightarrow$. (Конечно, на третьем этаже имеется еще много 'банальных' (изолированных) объектов.)

Этим примером мы хотели бы завершить рассказ о поликатегорном взгляде на кубильяжи. Подробности фомализма можно посмотреть в статье МанинаШехтмана [14] или Капранова-Воеводского [9, 15]. Само понятие поликатегории кратко обсуждается в книге [25] и более подробно - в книге [26]. Отметим еще статью [27, где на на поликатегорном языке обсуждается уравнение Замолодчикова.

\section{Дополнение 2. Связь с триангуляциями и посетом Тамари-Сташева}

Практически одновременно с введением кубильяжей в форме высшего посета Брюа появились (в работе Воеводского и Капранова [15]) посеты Тамари-Сташева, которые во многом параллельны посетам Брюа и тесно с ними связаны. На эту тему написано много работ, с которыми можно познакомиться по обзору [2].

Чтобы определить эти посеты, снова рассмотрим циклическую конфигурацию векторов $C(n, d)$. Концы этих векторов являются точками на гиперплоскости в $\mathbb{R}^{d}$, заданной уравнением $x_{1}=1$, то есть гиперплоскостью на 'высоте' 1 . Выпуклая оболочка этих точек $v_{1}, \ldots, v_{n}$ называется ииклическим политопом и обозначается $P(n, d)$; это пересечение зонотопа $Z(n, d)$ с гиперплоскостью, и его размерность равна $d-1$. При $d>2$ точки $v_{i}$ являются вершинами этого политопа.

Вместо кубильяжей зонотопа $Z(n, d)$ теперь интересуются триангуляциями политопа $P(n, d)$, то есть разбиениями на $(d-1)$-мерные симплексы, вершины которых лежат в множестве $\left\{v_{1}, \ldots, v_{n}\right\}$. При $d=2$ это разбиение отрезка $\left[v_{1}, v_{n}\right]$ на 
подотрезки, при $d=3$ - разбиение выпуклого $n$-угольника на $(n-2)$ треугольника, и так далее. Множество триангуляций обозначим $\mathbf{T S}(n, d)$.

Триангуляции во многом похожи на кубильяжи. Например, среди них есть 'стандартная' и 'антистандартная' триангуляции (или нижняя и верхняя триангуляции в терминологии [2]), которые получаются как ограничения соответствующих кубильяжей. В частности, этими триангуляциями исчерпываются множества $\mathbf{T S}(d+1, d)$. Замена стандартной триангуляции на антистандартную в $\mathbf{T} \mathbf{S}(d+1, d)$ называется повышающим флипом. Аналогичную вещь (повышающий флип) можно делать при любом $n$, если удается найти фрагмент вида стандартной триангуляции $\mathbf{T S}(d+1, d)$. Мы говорим, что $\mathcal{T} \leq \mathcal{T}^{\prime}$, если от $\mathcal{T}$ можно добраться до $\mathcal{T}^{\prime}$ серией повышающих флипов. Это дает структуру посета в множестве $\mathbf{T S}(n, d)$, называемую посетом Тамари-Сташева.

Пример 1. $d=2$. Задать тут триангуляцию - значит задать разбиение на отрезки отрезка $\left[t_{1}, t_{n}\right]$, причем так, чтобы концы отрезков были какие-то $t_{i}$. Любое подмножество в $\left\{t_{2}, \ldots, t_{n-1}\right\}$ задает такое разбиение на отрезки. Типичный повышающий флип состоит в замене двух последовательных отрезков $\left[t_{i}, t_{j}\right]$ и $\left[t_{j}, t_{k}\right]$ $\left(t_{i}<t_{j}<t_{k}\right)$ на отрезок $\left[t_{i}, t_{k}\right]$, то есть фактически удаление $t_{j}$ из подмножества. Мы видим, что посет Тамари-Сташева в этом случае (анти)изоморфен булевой решетке подмножеств множества $\{2, \ldots, n-1\}$.

Пример 2. Случай $d=3$ более интересен. Надо взять $n$ точек на параболе $y=x^{2}$, упорядоченных по возрастанию $x$; выпуклая оболочка этих точек дает циклический многоугольник $P=P(n, 3)$. Возьмем некоторую его триангуляцию и войдем (сверху) в треугольник со стороной $\left[v_{1}, v_{n}\right]$. После чего выйдем через любую из оставшихся сторон. Если при этом мы покидаем $P$, на этом путь заканчивается. Если попали в другой треугольник триангуляции, процесс продолжается - мы снова выходим через одну из двух других сторон этого треугольника. И так далее. В результате получается то, что называется плоским бинарным деревом (с $(n-2)$-мя некорневыми вершинами, соответствующими треугольникам триангуляции), см. рис. 20 и 21. В книге [28] (упр. 6.19) приведены еще 65 способов задания множества таких деревьев, среди которых стоит выдеить способ 'правильной' расстановки скобок в строке из $(n-1)$-й буквы. Этот способ использовал Тамари при определении его посета $\mathbf{T S}(n, 3)$.

Чтобы говорить не просто о множестве $\mathbf{T S}(n, 3)$, но и о частичном порядке на нем, надо конкретизировавать повышающие флипы. Они устроены так: надо взять фрагмент триангуляции, изображенный слева на рис. 19, и заменить диагональ $(i k)$ четырехугольника на другую диагональ $(j l)$.
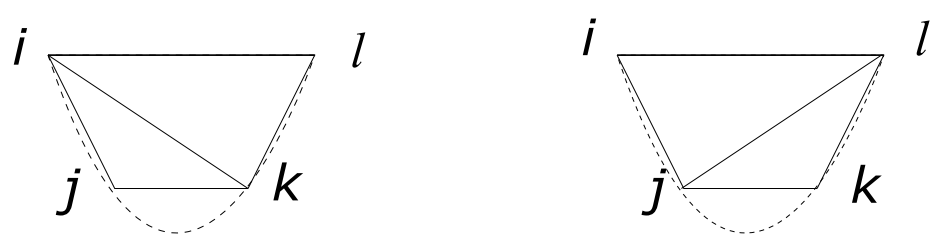

Рис. 19: флип триангуляции 
Ниже нарисованы пять трангуляций пятиугольника и соответствующие бинарные деревья.
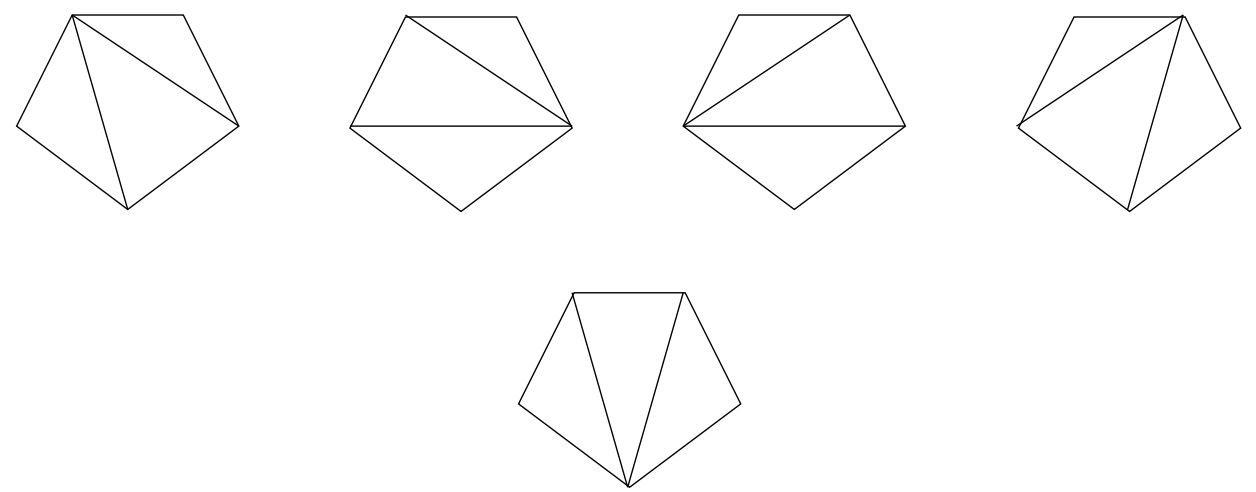

Рис. 20: пять триангуляций пятиугольника $P(5,2)$.
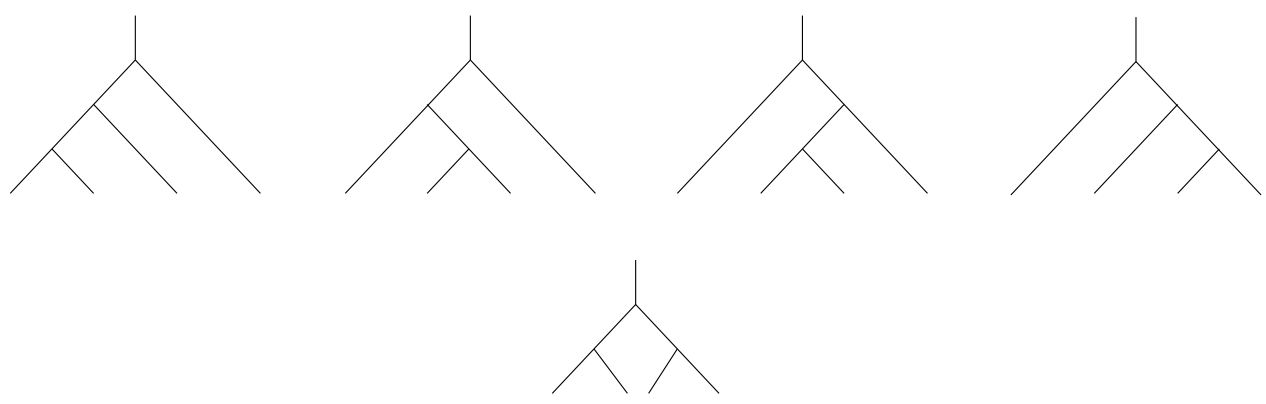

Рис. 21: соответствующие пять бинарных дерева.

Помимо очевидной аналогии с кубильяжами имеется более точная связь между ними. Пусть $\mathcal{Q}$ - кубильяж зонотопа $Z(n, d)$. Если мы пересечем этот зонотоп гиперплоскостью $x_{1}=1$, мы получим в точности политоп $P(n, d)$; сечения кубов той же гиперплоскостью дают триангуляцию политопа $P(n, d)$. Это дает естественное отображение

$$
\sec : \mathbf{Q}(n, d) \rightarrow \mathbf{T S}(n, d) .
$$

Триангуляция $\mathcal{T}=\sec (\mathcal{Q})$ дает полное представление о кубах, лежащих в 'основании' кубильяжа $\mathcal{Q}$ (то есть укорененных в 0 ), но мало что говорит о том, как лежат кубы выше. Поэтому нет оснований надеяться на инъективность отображения $s e c$, и уже простейшие примеры показывают неинъективность. В то же время есть веские основания надеяться, что верна следующпя

Гипотеза 1. Отображение sec: $\boldsymbol{Q}(n, d) \rightarrow \mathbf{T S}(n, d)$ сюрвективно.

Во всяком случае это так для $d \leq 3$. В случае $d=2$ это простое упражнение. Случай $d=3$ можно получить из работы [31, sec. 7] или из результатов [11]. 
Отметим, что в обзоре [2] (в пункте 8.3) также говорится про связь посетов Тамари и Брюа, но там обсуждается другое отображение. Что же касается отображения sec, то оно обсуждается в работе [33]. Там заявляется о сюръективности этого отображения, правда посет Тамари понимается несколько иначе, более ‘комбинаторно'. А вся их работа посвящена применению Тамари-посетов к солетонным решениям уравнения Кадомцева-Петвиашвили. Имеется еще несколько работ о связи уравнения КП и кубильяжей-триангуляций, из которых отметим самую последнюю 34]

Ометим еще, что отображение $s e c: \mathbf{Q}(n, d) \rightarrow \mathbf{T S}(n, d)$ согласовано с флипами. Если мы делаем флип в кубильяже $\mathcal{Q}$, то он может индуцировать флип в триангуляции $\sec (\mathcal{Q})$. Точнее, если мы делаем флип внутри некоторого капсида, укорененного в 0, мы получаем флип соответствующей триангуляции. Если же капсид расположен 'высоко', то флип кубильяжа не оказывает никакого воздействия на триангуляцию. В любом случае это дает, что отображение $s e c: \mathbf{Q}(n, d) \rightarrow \mathbf{T S}(n, d)$ согласовано со структурами посетов.

\section{Дополнение 3. Слабые мембраны}

Это дополнение непосредственно продолжает материал разделов 9-11. С другой стороны оно примыкает к тематике Дополнения 2 про триангуляции циклических политопов.

В разделе 6 вводилось понятие мембраны в кубильяже. Это $(d-1)$-мерный подкомплекс кубильяжа, который при проекции $\pi$ вдоль $d$-го координатного вектора $e_{d}$ биективно проектируется туда же, куда весь зонотоп $Z(n, d)$. Аналогично понимается и слабая мембрана. Это тоже некоторая $(d-1)$-мерная пленка в зонотопе $Z(n, d)$, биективно проектирующаяся туда же, куда и весь зонотоп. Но есть два отличия. Первое - эта пленка уже не является подкомплексом кубильяжа $\mathcal{Q}$, но подкомплексом некоторого измельчения этого кубильяжа. Второе - проектируем мы не вдоль вектора (направления) $e_{d}$, но вдоль вектора $e_{d}+\varepsilon e_{1}$, где $\varepsilon-$ небольшое положительное число. То есть мы глядим на зонотоп почти в направлении $e_{d}$, но чуть-чуть 'снизу'. Иначе говоря, проекция $\pi_{\varepsilon}$ вдоль вектора $e_{d}+\varepsilon e_{1}$ переводит точку $x=\left(x_{1}, x_{2}, \ldots, x_{d}\right) \in \mathbb{R}^{d}$ в $\pi_{\varepsilon}(x)=\left(x_{1}-\varepsilon x_{d}, x_{2}, \ldots, x_{d-1}\right) \in \mathbb{R}^{d-1}$.

Скажем более точно. Циклический зонотоп $Z=Z(n, d)$ как бы растет из точки 0 'вверх', до высоты $n$, и все его вершины, как и вершины кубильяжа $\mathcal{Q}$, находятся на целочисленной высоте. Рассечем и зонотоп $Z$, и все кубы кубильяжа $\mathcal{Q}$ 'горизонтальными' гиперплоскостями $H_{k}$, заданными уравнениями $x_{1}=k$, где $k$ пробегает целые число от 1 до $n-1$. В результате каждый куб $Q$ будет разрезан на $d$ частей , называемых кусками. Каждый кусок - это некоторый гиперсимплекс (в терминологии [29]). На рис. 22 показано рассечение трехмерного куба на три куска: нижний тетраэдр, октаэдр и верхний тетраэдр.

Получается разбиение зонотопа $Z$, но уже не на кубы, но на более мелкие куски-гиперсимплексы, которое мы обозначаем $\mathcal{Q} \equiv$ и называем измельчением кубильяжа $\mathcal{Q}$.

Измельченный кубильяж $\mathcal{Q} \equiv$ имеет те же вершины, что и $\mathcal{Q}$. Но нас больше 

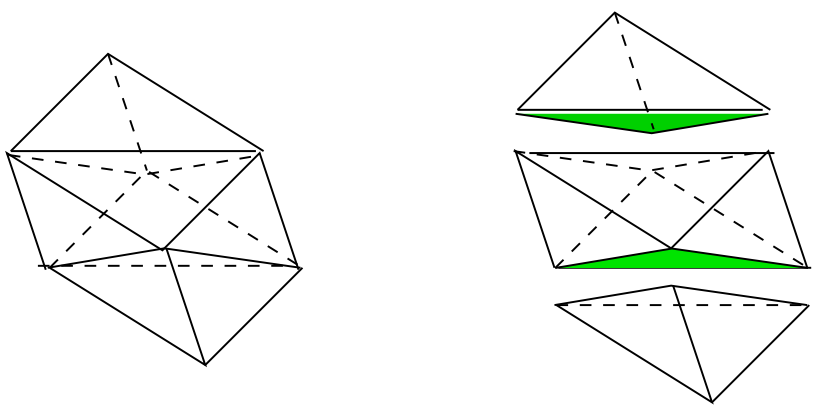

Рис. 22: куски

будут интересовать 'фасеты' $\mathcal{Q} \equiv$, или фасеты кусков. Они бывают 'вертикальные' (это куски фасет кубов кубильяжа $\mathcal{Q}$ ) и 'горизонтальные', лежащие в некоторой гиперплоскости $H_{k}$. (И те, и другие - гиперсимплексы, только теперь размерности $d-1$.) Большинство фасет куска $P$ вертикальные, и только две (или одна, если кусок - симплекс) горизонтальные.

Слабал мембрана (или $w$-мембрана) в кубильяже $\mathcal{Q}$ - это подкомплекс $\mathcal{W}$ измельчения $\mathcal{Q}$, который при проекции $\pi_{\varepsilon}$ вдоль вектора $e_{d}+\varepsilon e_{1}$, биективно (гомеоморфно) проектируется на зонотоп (меньшей размерности) $\pi_{\varepsilon}(Z)$. Обычные мембраны (из раздела 6, их можно называть сильными) могут пониматься как слабые. Как и сильные мембраны, слабые мембраны гомеоморфны $(d-1)$-мерному диску, край которого - это обод зонотопа $Z$ (относительно $\pi$ или $\pi_{\varepsilon}$ ), и они делят зонотоп $Z$ на две части - перед мембраной $\left(Z_{-}(\mathcal{W})\right)$ и после мембраны $\left(Z_{+}(\mathcal{W})\right)$; каждая из этих частей тоже разбита на куски. Проекции (при $\left.\pi_{\varepsilon}\right)$ клеток мембраны дают некоторое разбиение зонотопа $\pi_{\varepsilon}(Z)$ на плитки-гиперсимплексы, которое можно было бы назвать гиперкомби, по аналогии с понятием комби в размерности 2, [30, 31]. Это интересный объект, но мы (пока) не будем им заниматься.

Главное отличие слабых мембран от сильных в том, что первые могут иметь горизонтальные участки ('выступы' или 'балконы'), см. рис. 23 и 24.

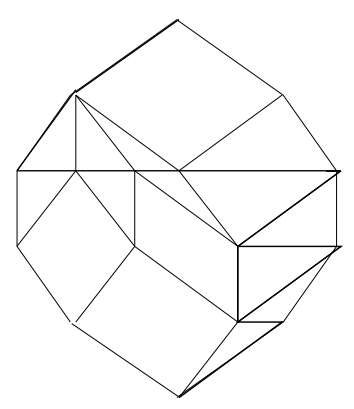

Рис. 23: слабая мембрана в ромбическом тайлинге; видны три балкона.

Простейшие примеры $w$-мембран (назовем их главными) получаются следующим образом. Пусть $\mathcal{Q}$ - кубильяж зонотопа $Z=Z(n, d)$. Мы идем (сверху вниз) сначала по передней (видимой) части границы $Z$ до уровня $k, 0 \leq k \leq n$. Затем 
идем горизонтально по этому уровню $k$ вплоть до невидимой (задней) части границы и наконец спускаемся до 0 по задней границе. На рис. 24 слева изображена главная $w$-мембрана (при $k=2$ ) для зоногона $Z(5,2)$, а справа - для зонотопа $Z(5,3)$ (вид спереди и немного снизу).
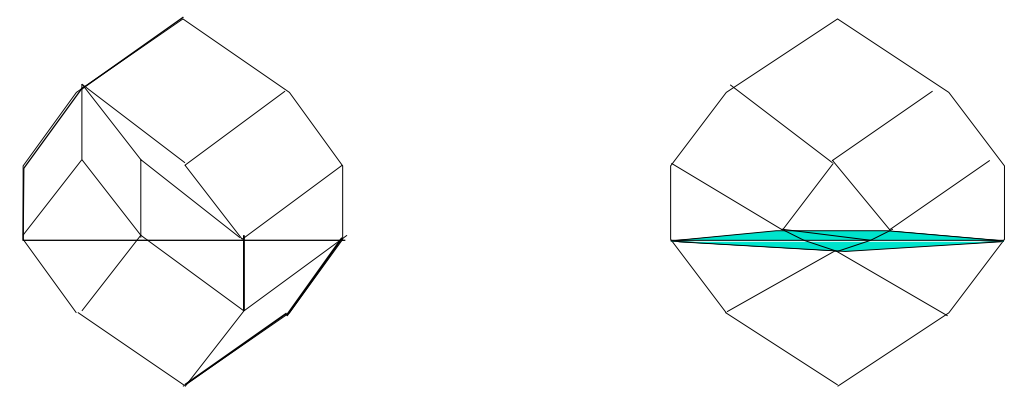

Рис. 24: простые мембраны.

Тут уступы имеются лишь на высоте 2 ; в общем случае уступы могут встречаться на любой высоте. Для тривиального кубильяжа куба $Z(d, d)$ все мембраны главные.

Отметим, что триангуляции циклического политопа из Дополнения 2 можно понимать как главные слабые мембраны (для $k=1$ ).

Основная вещь, о которой мы хотим рассказать - это аналог естественного порядка на множестве кусков измельченного кубильяжа $\mathcal{Q} \equiv$. Поступим в точности как в разделе 9. А именно, введем понятие непосредственного следования $\prec$ для кусков. Только проектируем (и, соотвественно, говорим о видимых и невидимых фасетах кусков) не отображением $\pi$, а чуть измененным отображением $\pi_{\varepsilon}$. При этом заметим следующее. Пусть кусок $P$ (лежащий в кубе $Q$ ) непосредственно предшествует куску $P^{\prime}$ (лежащему в кубе $\left.Q^{\prime}\right), P \prec P^{\prime}$. Тогда возможны два случая:

$1)$ куски $P$ и $P^{\prime}$ лежат на одном этаже (между гиперплоскостями $H_{k}$ и $H_{k+1}$ ), и тогда $Q \prec Q^{\prime}$; $P^{\prime}$.

2) куски $P$ и $P^{\prime}$ разделены гиперплоскостью $H_{k}$; тогда $Q=Q^{\prime}$, и $P$ лежит под

Это замечание помогает доказать следующий аналог Предложения 6:

Утверждение. Отношение $\prec н а \mathcal{Q} \equiv$ ациклично.

В самом деле, пусть $P_{1} \prec \ldots \prec P_{m}$ - некоторый направленный путь в $\mathcal{Q} \equiv$. И пусть $Q_{l}-$ куб кубильяжа $\mathcal{Q}$, содержащий кусок $P_{l}$. Тогда мы получаем направленный путь $Q_{1} \ldots Q_{m}$ в $\mathcal{Q}$, в котором соседние кубы либо связаны отношением $\prec_{\mathcal{Q}}$, либо совпадают. В силу Предложения 6 раздела 9 , если на этом пути встречается хоть раз отношение $\prec_{\mathcal{Q}}$, то $Q_{1}$ отлично от $Q_{m}$ и тем более $P_{1}$ отлично от $P_{m}$. Поэтому можно считать, что все $Q_{l}$ одни и те же и равны некоторому кубу $Q$. Тогда все куски $P_{l}$ - это куски этого куба $Q$. Теперь утверждение очевидно, потому что каждый следующий кусок лежит на этаж выше предыдущего.

Основываясь на этом утверждении, мы транзитивно замыкаем отношение $\prec$ и получаем отношение порядка $\preceq$ на $\mathcal{Q} \equiv$, которое снова называем естественным. 
И точно так же, как это делалось в разделе 11, можно ввести понятие стэка (или идеала) для этого порядка и отождествить слабые мембраны (для кубильяжа $\mathcal{Q}$ ) со стэками. Польза этого простого наблюдения в том, что стэк 'разбирается' путем удаления некоторого максимального (относитеольно порядка $\preceq$ ) элемента (куска). В терминах слабых мембран мы получаем понятие слабого (понижающего) флопа. Важное следствие состоит в том, что от любой слабой мембраны до 'минимальной' (и уже сильной) мембраны можно добраться серией таких слабых понижающих флопов. Кроме того, множество слабых мембран (в кубильяже $\mathcal{Q}$ ) является (как в Предложении 11) дистрибутивной решеткой.

Однако на этом аналогии частично обрываются, и ситуация начинает зависеть от четности/нечетности $d$. Вернемся к простейшему кубильяжу куба $Z(d, d)$. У измельчения такого куба имеется $d$ кусков. И почти у всех кусков (кроме средних) все вершины лежат на ободе этого куба. Как мы знаем из раздела 7, не на ободе находятся только две вершины, обозначенные там как $t$ и $h$ (хвост и голова). Обе эти вершины принадлежат либо одному 'среднему' куску (если $d$ нечетно), либо одному 'среднему' сечению (если $d$ четно, см. Рис. 44). И это сказывается на числе вершин слабой мембраны. Когда мы удаляем нецентральный кусок из груды $\mathcal{S}(\mathcal{W})$ домембранных кусков (делая соотвествующий понижающий флоп), то число вершин слабой мембраны не меняется. Однако когда мы удаляем центральные куски, положение усложняется. Удаление более верхнего куска (при четном $d$ ) приводит к увеличению числа вершин мембраны на 1, удаление более низкого к уменьшению на 1, а удаление единственного центрального куска (при нечетном d) не меняет числа вершин мембраны.

Вывод. При нечетном d слабые мембраны имеют одно и то же число вершин, равное $\left(\begin{array}{c}n \\ \leq d-1\end{array}\right)$. При четном d число вершин у слабых мембран может меняться (в каких пределах?).

Например, рис. 23 изображает тайлинг $Z(5,2)$ со слабой мембраной, у которой 12 вершин (вместо 'нормальных' шести).

А теперь вспомним Предложение 22 , которое говорит, что (при нечетном $d$ ) размер слабо $(d-2)$-разделенной системы в $[n]$ не превосходит $\left(\begin{array}{c}n \\ \leq d-1\end{array}\right)$. В 32$]$ доказывается, что при нечетном $d$ спектр любой слабой мембраны в $Z(n, d)$ является слабо $(d-2)$-разделенной системой (ср. с Предложением 15) . Также есть основания предполагать следующее.

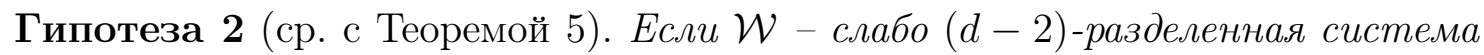
размера $\left(\begin{array}{c}n \\ \leq d-1\end{array}\right)$, то она реализуется как спектр некоторой слабой мембраной в зонотопе $Z(n, d)$.

Во всяком случае, для $d=3$ эта гипотеза верна.

\section{Дополнение 4. Доказательство ацикличности}

В этом дополнении мы собираемся доказать Предложение 6 об ацикличности отношения $\prec$ на множестве кубов кубильяжа $\mathcal{Q}$. На самом деле мы покажем большее 
- что отношение $\prec$ ациклично не только на множестве кубов фиксированного кубильяжа $\mathcal{Q}$, но на множестве $\mathcal{C}$ всех кубов всех кубильяжей. (Абстрактным) кубом называется произвольный $d$-мерный куб $C$ в зонотопе $Z(n, d)$, порожденный некоторым набором векторов в $\mathbf{V}$ и растущий из целой точки. Такой куб задается указанием своего корня $v(X)(X \subset[n])$ и своего типа $T \subset[n] ; T$ имеет размер $d$ и не пересекается с $X$. Как показано в Предложении 3 , такой куб вписывается в некоторый кубильяж. Множество всех кубов в $Z(n, d)$ обозначается $\mathcal{C}(n, d)$; очевидно, оно равно объединению множества всех кубов всех кубильяжей $Z(n, d)$.

На множестве $\mathcal{C}(n, d)$ введем бинарное отношение $\prec$ ровно так, как это было сделано в разделе 9 . Напомним, что $Q \prec Q^{\prime}$, если эти кубы $Q$ и $Q^{\prime}$ соседние по некоторой общей фасете $F$, причем эта фасета $F$ невидимая в $Q$ и видимая в $Q^{\prime}$. Предложение 6 следует из более сильного утверждения.

Теорема. Отношение $\prec$ на множестве $\mathcal{C}(n, d)$ ациклично.

Для доказательства теоремы мы выразим комбинаторно отношение $\prec$. Пусть куб $Q$ равен $(X, T)$, куб $Q^{\prime}-\left(X^{\prime}, T^{\prime}\right)$, и $Q \prec Q^{\prime}$. Обозначим через $F$ фасету, по которой эти кубы соседствуют. Комбинаторно эта фасета равна $(S, J)$, где $S$ корень фасеты, а $J$ - ее тип. Очевидно, $J=T \cap T^{\prime}$, размер ее равен $d-1$. Пусть $T=J i, T^{\prime}=J k$ для некоторых цветов $i$ и $k$. Вектор $v_{i}$ может либо входить в корень $F$, либо выходить из него. В первом случае $S=X i$, во втором $X=S i$. Аналогично с $k$. Так что возможен один из четырех случаев, изображенных на рисунке 25.

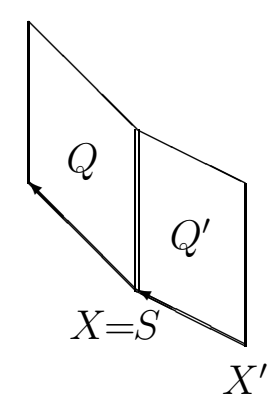

I

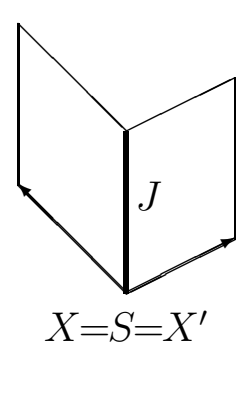

II

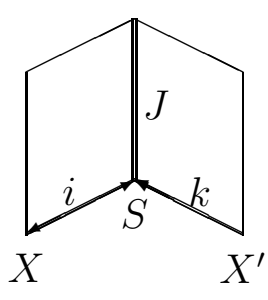

III

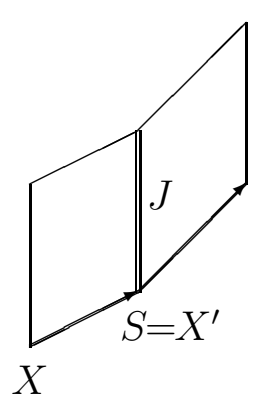

IV

Рис. 25: четыре варианта примыкания кубов к стенке $F$ типа $J$

Мы должны теперь выразить то, что фасета $F$ невидимая в кубе $Q$. Для этого будем временно считать, что корень фасеты $F$ расположен в нуле. Пусть $J=\left\{j_{1}<j_{2}<\ldots<j_{d-1}\right\}$; тогда линейное уравнение $\operatorname{det}\left(v_{j_{1}}, \ldots, v_{j_{d-1}}, \cdot\right)=0$ задает гиперплоскость, содержащую фасету $F$. То, что эта фасета невидимая в $Q$, означает, что вектор $v_{i}$ лежит в положительном полупространстве (если он входит в корень $F$ ) и в отрицательном полупространстве (если он выходит из корня $F$ ). То есть в случаях I и II $\operatorname{det}\left(v_{j_{1}}, \ldots, v_{j_{d-1}}, v_{i}\right)<0$, а в случаях III и IV этот определитель больше 0. 
Симметрично фасета $F$ видимая в кубе $Q^{\prime}$, если вектор $v_{k}$ лежит в положительном полупространстве в случаях II и IV, и в отрицательном полупространстве в случаях I и III. То есть определитель $\operatorname{det}\left(v_{j_{1}}, \ldots, v_{j_{d-1}}, v_{k}\right)$ должен иметь знак + или - соответственно.

Это возвращает нас к вопросу о знаках определителей, о котором мы рассказали в разделе 5 . А именно, точки $j_{1}<j_{2}<\ldots<j_{d-1}$ на отрезке $[n]$, делят этот отрезок на $d$ интервалов, которые удобно нумеровать справа налево. Нулевой интервал состоит из точек-чисел строго больших $j_{d-1}$, первый интервал - между $j_{d-2}$ и $j_{d-1}$ и так далее; последний интервал состоит из чисел, строго меньших $j_{1}$. Если число $j$ попадает в интервал с четным номером, то определитель $\operatorname{det}\left(v_{j_{1}}, \ldots, v_{j_{d-1}}, v_{j}\right)$ положителен; если с нечетным номером - отрицателен. Можно говорить, что число $j$ четно относительно $J$, если $j$ попадает в интервал с четным номером, и нечетно в противном случае.

Подведем итог. Пусть задана фасета $F$, представленная как пара $(S, J)$, и куб $Q=(X, T)$, имеющий $F$ своей фасетой, так что $T=J i$ для некоторого $i$, не принадлежащего $J$. Фасета $F$ невидима в кубе $Q$ тогда и толъко тогда, когда выполнено одно из двух:

a) $X=S$ и і нечетно относительно $J$;

b) $X=S-i$ и і четно относительно $J$.

Симметрично, пусть куб $Q^{\prime}=\left(X^{\prime}, T^{\prime}\right)$ имеет $F$ своей фасетой, так что $T^{\prime}=J k$. Фасета $F$ видима в кубе $Q$ тогда и только тогда, когда выполнено одно из двух:

a') $X^{\prime}=S$ и $k$ четно относительно $J$;

b') $X^{\prime}=S-k$ и $k$ нечетно относительно $J$.

Таким образом, $Q \prec Q^{\prime}$ тогда и только тогда, когда выполнены комбинации: a) и a') (случай II рисунка), или a) и b') (случай I рисунка), или b) и а') (случай IV) или b) и b') (случай III).

Итак, мы выразили комбинаторно отношение $\prec$. Теперь приступим к доказательству теоремы. Для этого разделим все множество $\mathcal{C}$ кубов на три группы, или уровня, $\mathcal{C}_{0}, \mathcal{C}_{1}$ и $\mathcal{C}_{2}$. $\mathrm{K}$ нулевому уровню $\mathcal{C}_{0}$ мы относим те кубы $(X, T)$, для которых цвет $n$ не входит ни в $X$, ни в $T$; к первому уровню ('перегородка цвета $n$ ') $\mathcal{C}_{1}$ мы относим те кубы, для которых $n$ принадлежит типу $T$; наконец, второй уровень $\mathcal{C}_{2}$ состоит из кубов, для которых $n \in X$. Следующее утверждение является ключевым.

Лемма. Уровенъ монотонно не убъвает относительно $\prec$.

Доказательство. Пусть $Q \prec Q^{\prime}$. Обозначим через $l$ и $l^{\prime}$ уровни $Q$ и $Q^{\prime}$, соотвественно. Мы должны проверить две вещи. Первая: если $l^{\prime}=0$, то и $l=0$. Вторая: если $l=2$, то и $l^{\prime}=2$.

Начнем со второго утверждения. Допустим, что $l=2$ (то есть $n$ принадлежит $X)$, но $n$ не принадлежит $X^{\prime}$. Так как в случае а') $X^{\prime}$ содержит $X$, этот случай не реализуем и имеет место случай $\left.\mathrm{b}^{\prime}\right)$. Но тогда $k=n$, а $n$ всегда четно, так что мы получаем противоречие.

Проверим теперь первое утверждение. Допустим, что $l^{\prime}=0$, тогда как $l>0$. Это значит, что $n$ не принадлежит ни $X^{\prime}$, ни $T^{\prime}$, и в то же время принадлежит либо $X$, либо $T$. Принадлежать $X$ цвет $n$ не может, как было только что показано. 
Таким образом, $n \in T=S i=\left(T^{\prime}-k\right) \cup\{i\}$. Так как $n$ не принадлежало $T^{\prime}$, то $i=n$. Но цвет $n$ всегда четен, так что мы попадаем в случай а), когда $X=S-i=S-n$. Значит $n$ принадлежит $S$ и тем более $X^{\prime}=S k$. Противоречие.

Приступим к доказательству теоремы. Оно ведется индукцией по $n$. При $n=d$ утверждение теоремы верно, поскольку имеется всего один куб. Будем считать, что $d<n$.

Предположим, что имеется циклический монотонный путь $Q_{0} \prec Q_{1} \prec \ldots \prec$ $Q_{N}=Q_{0}$. В силу предыдущей Леммы этот путь целиком располагается на одном из уровней, нулевом, первом или втором.

Если цикл проходит на уровне 0 , то он лежит в зонотопе $Z(n-1, d)$, что противоречит индуктивному предположению.

Если цикл лежит на втором уровне, то заменим каждый корень $X_{i}$ на $X_{i}-n$. Мы снова получаем циклический путь в зонотопе $Z(n-1 . d)$, что невозможно по индуктивному предположению.

Остается рассмотреть случай, когда циклический путь располагается на уровне 1. То есть, если $Q_{i}=\left(X_{i}, T_{i}\right)$ для $i=0, \ldots, N$, то $n \in T_{i}$ для всех $i$. В этом случае, заменяя каждое множество $T_{i}$ на 'редуцированное' множество $\widetilde{T}_{i}=T_{i}-n$, мы получим 'путь' $\widetilde{Q_{0}}, \ldots, \widetilde{Q_{N}}$ в $Z(n-1, d-1)$, где $\widetilde{Q_{i}}=\left(X_{i}, \widetilde{T}_{i}\right)$. Главное замечание состоит в том, что мы снова получаем цикл, хотя и противоположно ориентированный, что противоречит индуктивному предположению. Это замечание следует из приводимой ниже Леммы о реверсе.

Лемма о реверсе. Пусть для кубов $Q=(X, T)$ и $Q^{\prime}=\left(X^{\prime}, T^{\prime}\right)$ uз $\mathcal{C}(n, d)$ выполнено соотношение $Q \prec Q^{\prime}$. Предположим, что $n \in T, T^{\prime}$, и положим $\widetilde{T}=$ $T-n, \widetilde{T}^{\prime}=T^{\prime}-n$. Тогда для кубов $\widetilde{Q}=(X, \widetilde{T})$ и $\widetilde{Q^{\prime}}=\left(X^{\prime}, \widetilde{T}^{\prime}\right)$ в зонотопе $\widetilde{Z}=Z(n-1, d-1)$ выполнено противоположное соотношение $\widetilde{Q^{\prime}} \widetilde{\prec} \widetilde{Q}$, где $\widetilde{\gtrless}-$ соответствующее отношение на множестве $\mathcal{C}(n-1, d-1)$.

Доказательство. Пусть $J=T \cap T^{\prime}$; очевидно, что $J$ также содержит $n$. Положим $\widetilde{J}=J-n$. Причина реверса состоит в том, что в силу $J=\widetilde{J} n$ четность какоголибо цвета $i$ относительно множества $\widetilde{J}$ противоположна четности этого цвета относительно $J$.

\section{Список литературы}

[1] Rambau J. Triangulations of cyclic polytopes and higher Bruhat orders. Mathematika 44, 1997, 162-194.

[2] Rambau J., Reiner V. A survey of the higher Stasheff-Tamari orders. In Associahedra, Tamari Lattices and Related Structures (eds. F. Muller-Hoissen, J.M.Pallo, J. Stasheff), Birkhauser/Springer, 2012, pp. 351-390.

[3] Assosihedra, Tamati Lattices and Related Structures (eds. F. Muller-Hoissen, J.M. Pallo, and J. Stasheff), Springer, 2012. 
[4] Shephard G.C. Combinatorial properties of associated zonotopes. Can. J. Math., v. 26, No. 2, 1974, 302-321.

[5] Циглер Г.М. Теория многогранников. М,: МЦНМО, 2014. (Перевод Ziegler G. Lectures on polytopes, Springer-Verlag, N.Y., 1995.)

[6] Leclerc B., Zelevinsky A. Quasicommuting families of quantum Plücker coordinates. Amer. Math. Soc. Trans., Ser. 2, v. 181 (1998) 85-108.

[7] Данилов В.И., Карзанов А.В., Кошевой Г.А. Системы разделенных множеств и их геометрические модели. Успехи матем. наук, т. 65, вып. 4 (394) 2010, $132-217$.

[8] Манин Ю.И., Шехтман В.В. О высших порядках Брюа, связанных с симметрической группой. Функи. анализ и его приложения, 1986, т. 20, вып. 2, $74-75$.

[9] Воеводский В.А., Капранов М.М. Свободная $n$-категория, порожденная кубом, ориентированные матриоды и высшие порядки Брюа. Функи. анализ и его приложения, 1991, т. 25, вып. 1, 62-65.

[10] Ziegler G.M., Higher Bruhat orders and cyclic hyperplane arrangements. Topology, v. 32, No. 2, 1991, pp. 259-279.

[11] Galashin P. Plabic graphs and zonotopal tilings. Proc. London Math. Soc. 117 (4), 2018, 661-681.

[12] Galashin P., Postnikov A. Purity and separation for oriented matroids. arXiv:1708.01329[math.CO], 2017.

[13] Bailey G.D. Tilings of zonotopes: Discriminantal arrangements, oriented matroids, and enumeration, Minnesota Univ., 1997.

[14] Manin Yu., Schechtman V. Arrangemennts of hyperplanes, higher braid groups and higher Bruhat orders. In: Algebraic Number Theory - in Honour of $K$. Iwasawa, Advances Studies in Pure Math. 17, Academic Press, NY, 1989, pp. 289-308.

[15] Kapranov M.M., Voevodsky V.A. Combinatorial-geometric aspects of polycategory theory: pasting schemes and higher Bruhat orders. Cahiers de topologie et geometrie differentielle categoriques, t. 32, n. 1, 1991, 11-28.

[16] Bjorner A., Las Vergnas M, Sturmfels B., White N., Ziegler G.M. Oriented matroids. Cambridge Univ. Press, 1999.

[17] Athanasiadis C.A. Zonotopal subdivisioms of cyclic zonotopes, Geometriae Dedicata 86, 2001, 37-57

[18] Стенли Р. Перечислительная комбинаторика, т. 1. Москва, Мир, 1990 
[19] Felsner S., Ziegler G.M. Zonotopes associated with higher Bruhat orders. Discrete Mathematics 241, 2001, pp. 301-312.

[20] Thomas H. Maps between higher Brughat orders and higher Stasheff-Tamari posets. In Formal Power Series and Algebraic Combinatorics Conference Linköping, Sweden, 2003.

[21] Las Vergnas M. Extensions ponctuelles compatibles d'une geometrie combinatoire. C.R. Acad. Sci. Paris, Ser. A-B, 286(21), A981-A984, 1978.

[22] Felsner S., Weil H. A theorem on higher Bruhat orders. Discrete Comput. Geom. 23, 2000, pp. 121-127.

[23] Edelman P.H., Reiner V. Free arrangements and rhombic tilings. Discrete Comput. Geom., 16 (1996) 307-340.

[24] Oppermann A., Thomas H. Higher dimensional cluster combinatorics and representation theory. arXiv:1001.5437[math.RT], 2011.

[25] Маклейн С. Категории для работающего математика. Москва, Физматлит, 2004.

[26] Lurie J. Higher topos theory. Princeton Univ. Press, 2009.

[27] Kapranov M.M., Voevodsky V.A. 2-categories and Zamolodchikov tetrahedra equations. Proc. of Symposia in Pure Math. v. 56, part. 2, 1994.

[28] Стенли Р. Перечислительная комбинаторика, т.2. Москва, Мир, 2005.

[29] Гельфанд И.М., Серганова В.В. Комбинаторные геометрии и страты тора на однородных компактных многообразиях. УМН, 1987, т. 42, вып. 2(254), 107143.

[30] Danilov V.I., Karzanov A.V., Koshevoy G.A. Combined tilings and separated setsystems. Selecta Mathematica, v. 23, 2017, 1175-1203.

[31] Danilov V.I., Karzanov A.V., Koshevoy G.A. On interrelations between strongly, weakly and chord separated set-systems (a geometric approach). arXiv:1805.09595[math.CO], 2018.

[32] Danilov V.I., Karzanov A.V., Koshevoy G.A. The weak separation in higher dimensions. Manuscript, 2019.

[33] Dimakis A., Müller-Hoissen F. KP line solitons and Tamari lattices. In Associahedra, Tamari Lattices and Related Structures (eds. F. Muller-Hoissen, J.M.Pallo, J. Stasheff), Birkhauser/Springer, 2012, pp. 391-424.

[34] Karpman R., Kodama Y. Triangulations and soliton graphs for totally positive Grassmanian. arXiv:1808.01587[nlin.SI] 2018. 


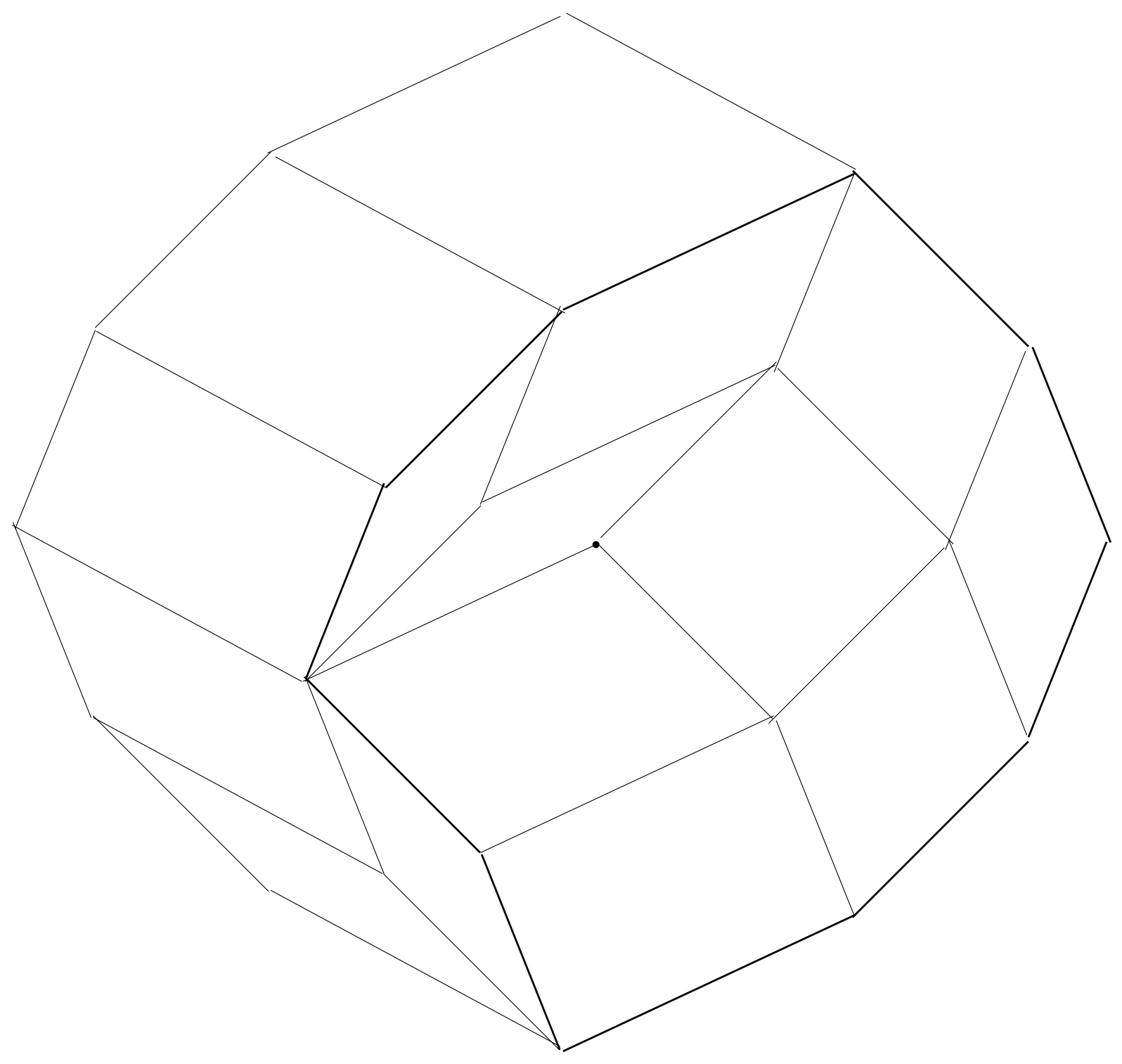

